\title{
Asymmetric All-Pay Contests with Heterogeneous Prizes*
}

\author{
Jun $\mathrm{Xiao}^{\dagger}$
}

November 24, 2015

\begin{abstract}
This paper studies complete-information, all-pay contests with asymmetric players competing for heterogeneous prizes. In these contests, each player chooses a performance level or "score". The first prize is awarded to the player with the highest score, the second - less valuable - prize to the player with the second highest score, etc. The players are asymmetric as they incur different scoring costs, and they are assumed to have ordered marginal costs. The prize sequence is assumed to be either geometric or quadratic. We show that each such contest has a unique Nash equilibrium, and we exhibit an algorithm that constructs the equilibrium. Then, we apply the results to study the issue of tracking in schools and the optimality of winner-take-all contests.
\end{abstract}

JEL classification: D44, D72

Keywords: all-pay, asymmetric, contest, heterogeneous

\section{Introduction}

Asymmetric players and heterogeneous prizes are predominant in contests. For example, students with different intelligence levels compete for different grades, athletes with different abilities compete for different medals, and employees with different experience compete for different promotion opportunities. The key characteristics common to these contests are: heterogeneous prizes awarded solely on the basis of relative performance; participants with possibly different abilities; and sunk costs of participants' investments.

Moreover, the prize sequences in such contests are usually convex - the difference between higher prizes is greater than the difference between lower ones. For instance, the difference in returns to higher education between top-ranked students and average students is typically much higher than

${ }^{*}$ First draft: November 2011. I would like to thank Vijay Krishna for his guidance, Ethem Akyol, Filippo Balestrieri, Peter Bardsley, Kalyan Chatterjee, Yeon-Koo Che, Nisvan Erkal, Edward Green, Simon Loertscher, Claudio Mezzetti, Benny Moldovanu, John Morgan, Roberto Raimondo, Ron Siegel, Neil Wallace, the reading committee at the University of Melbourne and seminar participants for comments and discussion. Our editor and a group of referees greatly helped to improve the paper's content and exposition.

${ }^{\dagger}$ Department of Economics, University of Melbourne. E-mail: jun.xiao@unimelb.edu.au. 
the difference between the average students and low-ranked students. Convex prize structures are also common in sports. The winner of the 2013 US Open tennis tournament was awarded a prize of $\$ 2.6$ million. The runner-up won $\$ 1.3$ million whereas those in the joint third position - the losing semi-finalists - won $\$ 650$ thousand each. The prize for a particular rank was roughly twice the prize for the next rank. ${ }^{1}$

This paper presents a contest model with the combination of asymmetric players and convex prize sequences. Specifically, we study complete-information all-pay contests in which participants with different abilities compete for heterogeneous prizes. The different abilities are represented by different costs of performance, and the marginal costs are ordered (a stronger participant's marginal cost is higher than that of a weaker participant at any performance level). The prize sequence is either geometric (the ratio of successive prizes is a constant, like at the US Open tennis tournament) or quadratic (the second-order differences are a positive constant). Each player chooses a costly performance level or "score". The player with the highest performance receives the highest prize, the player with the second-highest performance receives the second highest prize, and so on (the prizes may be allocated randomly in the case of a tie). A player's payoff is his winnings (if any) minus his cost of performance. The cost is incurred regardless of whether the player wins a prize or not.

Our main result is that such contests have a unique Nash equilibrium. Moreover, we provide an algorithm to construct the equilibrium. Both the uniqueness and construction rely on the algorithm. A key feature of the algorithm is that a weaker player's equilibrium payoff can be determined by examining his best response to the strategies in a smaller contest in which only players stronger than him participate. This feature, formally stated in Proposition 5, allows us to start with a set of stronger players and determine the equilibrium payoff of the next strongest player, and therefore derive his strategy. Then, we can move on to determine the equilibrium payoff of another, still weaker player.

These results allow us to tackle several challenges. If two or more participants have the same cost functions, there may be multiple equilibria. Moreover, prize allocation and total expected performance may differ across equilibria (see Example 2 below). In many applications, it is the planner's objective to maximize the total expected performance, so multiple equilibria make it challenging to compare different contest formats. In contrast, our result shows a unique equilibrium if no two participants have the same cost function. Therefore, the uniqueness of equilibrium is a generic property. As a result, if the multiplicity occurs, we can select a unique equilibrium as the limit of a sequence of unique equilibria in contests with slightly perturbed costs.

The algorithm that explicitly constructs the equilibrium allows us to study two applications. First, suppose a school wants to assign a group of students to different classrooms in order to maximize their total performance. Should the school separate the students according to their abilities - a practice known as "tracking" - so that high ability students are grouped together,

\footnotetext{
${ }^{1}$ Similarly, at the 2014 US Open golf tournament, the winner received $\$ 1.62$ million, the two players tied for runner-up received $\$ 789$ thousand each - the average of the prizes for positions 2 and 3 , the five players tied for third-place received $\$ 326$ thousand each - the average of the prizes for positions 4 to 8 .
} 
or should the school have mixed classrooms in which students of different abilities are grouped together? Tracking seems to have several advantages. It makes the high ability students compete against each other, so it encourages them to work harder. Moreover, if grouped together, students of moderate ability may also work harder since the absence of high ability competitors increases their chances of obtaining a higher relative rank. Our analysis shows, somewhat surprisingly, that mixing results in higher total expected performance if and only if the prize sequence is convex enough. Therefore, from a purely incentive viewpoint, mixing could be superior to tracking.

Second, consider a situation in which the planner of a contest has a fixed total amount as prize money and wants to maximize the total performance. Is a winner-take-all prize structure optimal, or should the total amount be split into two or more prizes? Barut and Kovenock (1998) show that, with complete information and symmetric participants, full surplus extraction can be obtained with many prize structures including the winner-take-all structure. ${ }^{2}$ In a different setting with incomplete information, Moldovanu and Sela (2001) show that, if participants are ex ante symmetric and their costs are linear, a winner-take-all prize structure is indeed optimal. ${ }^{3}$ In contrast, we show below that, if the participants are asymmetric, the optimality of the winnertake-all structure may be eliminated. In particular, we characterize a situation in which geometric or quadratic prize sequences with multiple prizes can result in higher total expected performance than the winner-take-all structure.

Literature There is a substantial literature on all-pay contests and, closely related, all-pay auctions. Since a comprehensive survey of the whole field can be found in the book of Konrad (2009), in what follows, we discuss only the work that is directly related to this paper.

Complete-information all-pay auctions can be shown to be isomorphic to all-pay contests. ${ }^{4}$ Among these auctions, the case with a single prize are analyzed by Baye et al. (1996). The case of multiple prizes with symmetric players is studied by Barut and Kovenock (1998). Both papers provide conditions for a unique equilibrium and demonstrate the possibility of multiple - a continuum of - equilibria.

The various studies of all-pay contests with multiple prizes differ in two dimensions: the prize sequence $v^{1} \geq v^{2} \geq \ldots \geq v^{m}$, and the players' cost functions $C_{i}(s)$. Clark and Riis (1998) study contests in which prizes are homogeneous while players are asymmetric and have linear (constant marginal) costs. They show that such contests have a unique equilibrium. Assuming homogeneous prizes, Siegel (2010) also establishes equilibrium uniqueness, and he allows very general, possibly nonlinear, cost functions. Bulow and Levin (2006) consider heterogeneous prizes. In their paper, the prize sequence is assumed to be arithmetic, that is, the differences in successive prizes are a

\footnotetext{
${ }^{2}$ See Theorem 3 of Barut and Kovenock (1998).

${ }^{3}$ It should be noted that their model is of incomplete information whereas our model is of complete information.

${ }^{4}$ In an all-pay auction, the players are only characterized by their valuations; in an all-pay contest with one prize, the players are only characterized by their costs of scores. The isomorphism means that, given an equilibrium in one model, we can construct one and only one equilibrium in the other.
} 


\begin{tabular}{lcc} 
& PRIZE SEQUENCE & COSTS \\
\hline Baye et al. (1996) & Single prize & Different linear \\
Barut and Kovenock (1998) & Arbitrary & Symmetric linear \\
Clark and Riis (1998) & $\begin{array}{c}\text { Homogeneous } \\
v^{k}=v^{k+1} \\
\text { Arithmetic }\end{array}$ & Different linear \\
Bulow and Levin (2006) & $\begin{array}{c}v^{k}-v^{k+1}=\beta \\
\text { Homogeneous } \\
v^{k}=v^{k+1} \\
\text { Arithmetic } \\
v^{k}-v^{k+1}=\beta\end{array}$ & Different linear \\
Siegel (2010) & Quadratic & Arbitrary \\
González-Díaz and Siegel (2013) & Different ordered nonlinear \\
This paper & $\begin{array}{c}\text { Geometric } \\
v^{k}=\alpha v^{k+1}\end{array}$ & Different ordered nonlinear \\
This paper & & \\
\hline
\end{tabular}

Table 1: All-Pay Contest Models

constant; the costs are assumed to be linear and may differ across players. ${ }^{5}$ Again, they obtain a unique equilibrium. González-Díaz and Siegel (2013) extend the work of Bulow and Levin (2006) by allowing a particular form of nonlinear costs. However, none of the papers consider convex prize sequences - the distinguishing feature of this paper. Olszewski and Siegel's (2013) study on large contests is also relevant. They characterize the limit equilibrium if the number of participants becomes large in various contests including the ones in this paper. Table 1 provides an "at-a-glance" comparison of the various models along the two dimensions.

Our analysis relies on an algorithm that constructs the equilibrium. Similar approaches have been used in the literature (e.g. Bulow and Levin 2006 and Siegel 2010, 2014). However, the equilibrium in this paper cannot be constructed by the existing methods. This is a result of two equilibrium properties. First, the mixed strategies in the equilibrium may have gaps in their supports. In contrast, the equilibrium strategies always have interval supports in the contests studied by Bulow and Levin (2006), so their algorithm does not apply here. Second, the highest score in a mixed strategy's support may vary across players in the equilibrium. In contrast, the highest scores are the same in the contests with homogeneous prizes, which are studied as an application by Siegel (2010). Because of this difference, we cannot obtain equilibrium payoffs as Siegel (2009) does. ${ }^{6}$ Since the algorithm of Siegel (2010) starts with equilibrium payoffs, it does not apply to our setting either.

The remainder of the paper is organized as follows. Section 2 introduces the general model and main results. Section 3 discusses the unique equilibrium and its construction for linear costs. Section 4 then extends the analysis to nonlinear costs. Section 5 applies our results to study two

\footnotetext{
${ }^{5}$ Figure 2 demonstrates that, if the prize sequence is not arithmetic, there can be a gap in the support of a player's equilibrium mixed strategy, so their algorithm does not apply. Hence, the assumption of multiplicative form of match surplus is important for their analysis.

${ }^{6}$ Because the prizes are heterogeneous, players' reach (Siegel 2009) is not defined in the context of this paper, so his characterization of equilibrium payoffs does not apply here.
} 
applications, and Section 6 concludes.

\section{Model}

Consider a complete-information, all-pay contest with $n \geq 2$ players. Because we already understand the equilibrium if $n=2$, we focus on the case with $n \geq 3 .^{7}$ Let $\mathcal{N}=\{1,2, \ldots, n\}$ be the set of players. There are $n$ monetary prizes in amounts $v^{1}>v^{2}>\ldots>v^{m}>v^{m+1}=\ldots=v^{n}=0$ to be awarded, and $m$ of them are positive, where $m \leq n$. Let $\left\{v^{k}\right\}_{k=1}^{n}$ denote the prize sequence. The players choose their scores $s_{i} \geq 0$ simultaneously and independently. The player with the highest score wins the highest prize, $v^{1}$; the player with the second-highest score wins the second highest prize, $v^{2}$; and so on. In the case of a tie, prizes are awarded in a way, perhaps randomly, such that no tying player always loses. ${ }^{8}$ The cost of score $s$ for player $i$ is $C_{i}(s)$, where function $C_{i}$ is increasing, differentiable and satisfies $C_{i}(0)=0$. Player $i$ 's payoff is $v^{k}-C_{i}\left(s_{i}\right)$ if he chooses score $s_{i}$ and wins the $k$ th prize. All players are risk-neutral.

Assumption A1: The players have ordered marginal costs. That is, $0<C_{1}^{\prime}(s)<\ldots<C_{n}^{\prime}(s)$ for all $s \geq 0$.

Thus, the players are strictly ordered according to "ability". In particular, player 1 is the strongest in the sense that his marginal cost is the lowest, player 2 is the second strongest, etc. If $i<j$, We will say that player $i$ is stronger than player $j$ and equivalently that player $j$ is weaker than player $i$. Note that both linear and nonlinear costs could have ordered marginal costs.

Assumption A2: $\left\{v^{k}\right\}_{k=1}^{n}$ is a geometric prize sequence (GPS) or a quadratic prize sequence (QPS). In a GPS, we have $m=n$ (so $v^{n}>0$ ) and $v^{k}=\alpha v^{k+1}$ for $k<n$ where $\alpha>1$ is a constant; in a QPS, we have $\left(v^{k}-v^{k+1}\right)-\left(v^{k+1}-v^{k+2}\right)=\beta$ for $k=1, \ldots, m-1$ where $\beta \geq 0$ is a constant.

Under Assumption A2, $\left\{v^{k}\right\}_{k=1}^{n}$ is a weakly decreasing sequence with $v^{1}$ being the highest and $v^{n}$ the lowest. In a GPS, the number of prizes equals the number of players, so the sequence is characterized by two parameters, $v^{n}$ and $\alpha$. If we normalize $v^{n}=1$, then $v^{k}=\alpha^{n-k}$. A QPS is characterized by three parameters, $m, v^{m}$ and $\beta$. By varying the parameters, we can change the number of positive prizes, the slope and convexity of the sequence. If we normalize the lowest positive prize $v^{m}=1$ in a QPS, we have $v^{k}=(m-k+1)[(m-k) \beta+2] / 2$ for $k=1, \ldots, m+1$.

A profile of strategies constitutes a Nash equilibrium if each player's (mixed) strategy assigns a probability of one to the set of his best responses against the strategies of other players. Our main result is:

Theorem 1 Every all-pay contest with a geometric or quadratic prize sequence and ordered marginal costs has a unique Nash equilibrium.

\footnotetext{
${ }^{7}$ For instance, Siegel (2010) provides equilibrium charaterization for the case of two players with asymmetric costs.

${ }^{8}$ In many tournaments (for example, in golf), ties are resolved by a sharing of the prizes. As an example, if players $i$ and $i^{\prime}$ tie with the second-highest score, then each receives $\left(v^{2}+v^{3}\right) / 2$. Our formulation allows this kind of sharing.
} 
The theorem's proof for the case of linear costs is in Section 3, and it is extended to nonlinear costs in Appendix D. For clarity of exposition, the proofs of most propositions are not included in the main text, and can be found in the appendix of the corresponding section.

We can use Theorem 1 to tackle challenges caused by multiple equilibria. If some players have the same cost function, there could be multiple equilibria. This could result in challenges for the applications in Section 4 because prize allocation or total expected score could be different across the equilibria. Example 2 demonstrates such equilibria and illustrates how we can apply the theorem to select a unique equilibrium. Corollary 1 discusses the selection for the general case.

\section{$3 \quad$ Linear Costs}

Because the analysis for linear costs is simpler, we first consider linear costs in this section, and extend the results to nonlinear costs in Section 4. Denote the players' marginal costs as $0<c_{1}<$ $c_{2}<\ldots<c_{n}$. We first provide a sequence of equilibrium properties in Section 3.1, then introduce an algorithm and discuss its properties in Section 3.2. In Section 3.3, we combine the equilibrium properties and algorithm properties to show that the equilibrium is unique and is constructed by the algorithm.

\subsection{Equilibrium Properties}

We begin with the observation that a contest with GPS or QPS has at least one equilibrium. Siegel (2009) establishes the existence of equilibrium if the prizes are homogeneous, and his proof is readily adapted to include the kinds of prize sequences considered here. In the interests of space, we omit the minor details. ${ }^{9}$

The following properties of an all-pay contest are either well-known or easily derivable from known results in the literature. ${ }^{10}$ In any equilibrium:

- (Participation) Every player $i>m+1$ assigns probability one to score 0 .

- (No Atom) No player assigns positive probability to a particular positive score.

- (No Aggregate Gaps) Each score between 0 and the maximum score is contained in the common support of at least two players' mixed strategies.

Since the second property excludes pure strategy equilibria, a Nash equilibrium (henceforth, equilibrium) consists of a set of cumulative distribution functions $\left\{G_{i}^{*}\right\}_{i=1}^{n}$, where $G_{i}^{*}$ represents $i$ 's mixed strategy. Throughout the paper, we use $G_{i}^{*}$ to represent the cumulative distribution function and $G_{i}^{*}(s)$ to represent the probability of player $i$ 's score being no higher than $s$.

\footnotetext{
${ }^{9}$ If we replace "the probability of winning" by "probability of winning one prize", the proofs for the Tie Lemma and Zero Lemma of Siegel (2009) apply here. If we replace $i$ 's reach by $v^{1} / c_{i}$, the proof of Corollary 1 of Siegel (2009) also applies here.

${ }^{10}$ The first property is proved in Appendix A by Lemma 2. The proofs of the second and third properties are similar to the argument of Bulow and Levin (2006), and are omitted.
} 
We need to introduce some definitions before discussing other equilibrium properties. The support of a mixed strategy is the smallest closed set whose complement has probability zero. Let $\underline{s}_{i}^{*}$ and $\bar{s}_{i}^{*}$ be the minimum and maximum scores in the support of equilibrium strategy $G_{i}^{*}$. Given an equilibrium, define $\mathcal{P}^{*}(s)=\left\{i \mid \underline{s}_{i}^{*} \leq s \leq \bar{s}_{i}^{*}\right\}$ and define $\mathcal{A}^{*}(s)$ as the set of players with increasing $G_{i}^{*}$ over interval $[s, s+\varepsilon)$ or $(s-\varepsilon, s]$ for some $\varepsilon>0$. Therefore, $\mathcal{A}^{*}(s) \subseteq \mathcal{P}^{*}(s)$. We refer to $\mathcal{A}^{*}(s)$ as the set of active players at $s$, and $\mathcal{P}^{*}(s)$ as the set of participating players at $s$. If there is a gap containing $s$ in the support of $G_{i}^{*}$, then $i \in \mathcal{P}^{*}(s)$ but $i \notin \mathcal{A}^{*}(s)$. Assumptions A1 and $\mathrm{A} 2$ are important for the participation property above and for the three equilibrium properties below.

Proposition 1 (Nested Gaps) Given any equilibrium, suppose $i, j \in \mathcal{P}^{*}(s)$ and $i<j$. Then, $i \in \mathcal{A}^{*}(s)$ if $j \in \mathcal{A}^{*}(s)$.

Suppose players $i, i+1 \in \mathcal{P}^{*}(s)$ and suppose $G_{i}^{*}$ has a gap $\left(s^{\prime}, s^{\prime \prime}\right)$ in its support. Then, Proposition 1 implies that the support of $G_{i+1}^{*}$ has a gap that contains $\left(s^{\prime}, s^{\prime \prime}\right)$. That is, the gap of a stronger player is nested in a gap of a weaker player. Figure 1 schematically illustrates the supports of equilibrium strategies in a contest with 7 positive prizes and 9 players. As we can see in the figure, the gaps are nested. One of the challenges to construct an equilibrium is to find the gaps in the supports of mixed strategies. Because the gaps are nested, we only need to search gaps of a stronger player within the gaps of a weaker player. Therefore, Proposition 1 simplifies the search of gaps.

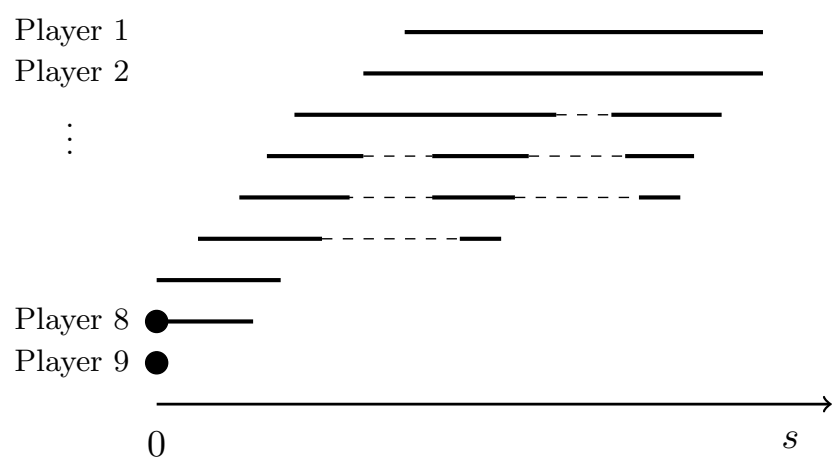

Figure 1: Nested Gaps

Proposition 2 below implies that the equilibrium strategies are ordered, and Proposition 3 implies that their derivatives are also ordered. As a result, for $i<j, G_{i}^{*}(s)$ decreases faster than $G_{j}^{*}(s)$ as $s$ decreases.

Proposition 2 (Stochastic Dominance) If $i<j$, then $G_{i}^{*}(s) \leq G_{j}^{*}(s)$ for $s \geq 0$. That is, the stronger player has higher scores than a weaker one in the sense of first order stochastic dominance.

Proposition 3 (Ordered Densities) Suppose no player's equilibrium strategy has s on the boundary of its support. Then, $G_{i}^{*}$ is differentiable at $s$ for any $i$, and $G_{i}^{* \prime}(s) \geq G_{j}^{* \prime}(s)$ if $i<j$. 
The example below illustrates the properties in Propositions 2 and 3.

Example 1 Consider a contest with three players. The prize sequence is quadratic and has two positive prizes worth $v^{1}=4$ and $v^{2}=1$ respectively. The marginal costs are $c_{1}=4$ for player 1 , $c_{2}=6$ for player 2 , and $c_{3}=7$ for player 3 . The equilibrium payoffs are $u_{1}^{*}=1.72$ for player $1, u_{2}^{*}=0.58$ for player 2 and $u_{3}^{*}=0$ for player 3 . Figure 2 plots the equilibrium strategies. The construction of the strategies is discussed in Section 3.2.

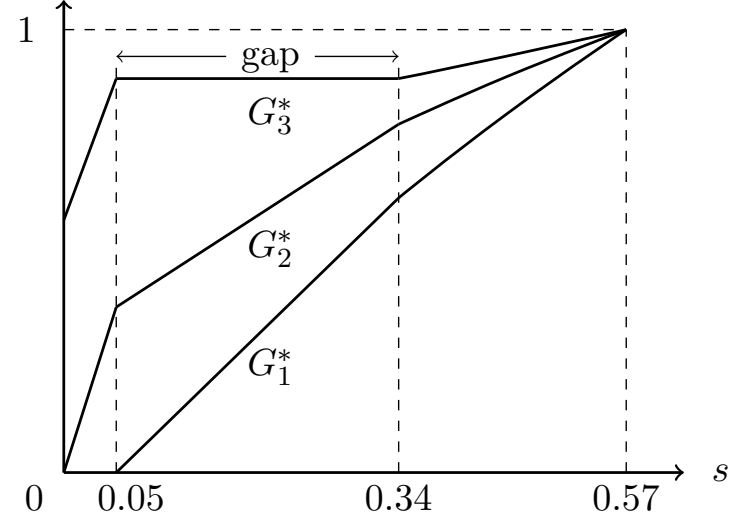

Figure 2: Equilibrium Strategies

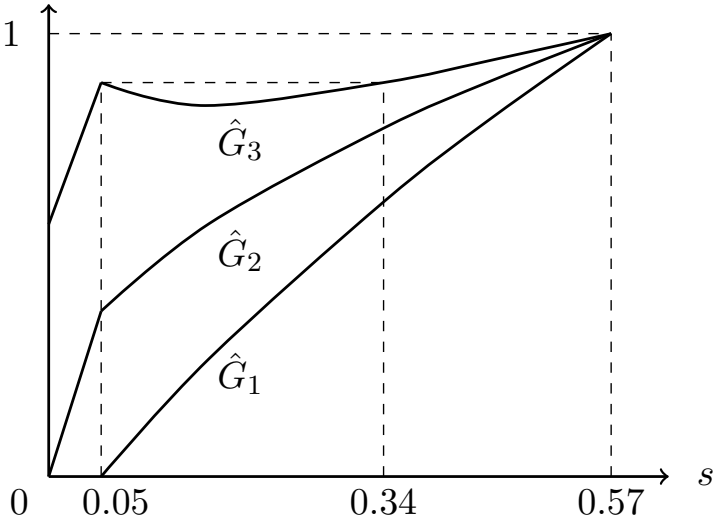

Figure 3: Pseudo Strategies

As we can see in Figure 2, there is a gap $(0.05,0.34)$ in the support of $G_{3}^{*}$. Why does player 3 not mix over scores in the gap? This is because the other players are competing so aggressively that his payoff at any score in the gap is negative. In addition, notice that $G_{2}^{*}$ in Figure 2 is not differentiable at the score 0.05 or 0.34 , which is on the boundary of the support of $G_{3}^{*}$. This is why Proposition 3 excludes the boundary scores. Propositions 2 and 3 also simplify the equilibrium construction. In particular, an implication of Proposition 2 is that the upper boundaries and lower boundaries of the supports are ranked in any equilibrium. That is, $\underline{s}_{i}^{*} \leq \underline{s}_{j}^{*}$ and $\bar{s}_{i}^{*} \leq \bar{s}_{j}^{*}$ if $i<j$. As a result, when searching for the upper boundaries, we can start from high scores and move towards lower ones. Then, we can find the upper boundaries from the strongest player to the weakest player. Proposition 3 establishes the piecewise differentiability of the mixed strategies, so we can characterize the strategies as a local solution to an ordinary differential equation system.

In order to discuss the remaining equilibrium properties, we need to introduce the concept of pseudo strategies. In addition to the current section, pseudo strategies are also repeatedly used in the algorithm to construct the equilibrium in Section 3.2. We first define pseudo strategies in the context of Example 1, then present the definition for the general case. 
Example 1 (continued) Consider the contest in Example 1. Recall that Figure 2 illustrates an equilibrium with mixed strategies $G_{1}^{*}, G_{2}^{*}$ and $G_{3}^{*}$. For $s \in[0.34,0.57]$, we must have

$$
\begin{aligned}
& G_{2}^{*}(s) G_{3}^{*}(s) v^{1}+\left[G_{2}^{*}(s)\left(1-G_{3}^{*}(s)\right)+\left(1-G_{2}^{*}(s)\right) G_{3}^{*}(s)\right] v^{2}-c_{1} s=u_{1}^{*} \\
& G_{1}^{*}(s) G_{3}^{*}(s) v^{1}+\left[G_{1}^{*}(s)\left(1-G_{3}^{*}(s)\right)+\left(1-G_{1}^{*}(s)\right) G_{3}^{*}(s)\right] v^{2}-c_{2} s=u_{2}^{*} \\
& G_{1}^{*}(s) G_{2}^{*}(s) v^{1}+\left[G_{1}^{*}(s)\left(1-G_{2}^{*}(s)\right)+\left(1-G_{1}^{*}(s)\right) G_{2}^{*}(s)\right] v^{2}-c_{3} s=u_{3}^{*}
\end{aligned}
$$

The first equation means that the mixed strategies $G_{2}^{*}$ and $G_{3}^{*}$ of players 2 and 3 make player 1 indifferent among different scores. The other two equations are analogous.

However, if $s \in(0.05,0.34)$, there is a gap in the support of $G_{3}^{*}$ that contains $s$, so the solution to equations (1) to (3) is different from the equilibrium strategies. In order to distinguish from the equilibrium strategies, we call the solution $\hat{G}_{1}(s), \hat{G}_{2}(s), \hat{G}_{3}(s)$ to the equations as pseudo strategies. To complete the definition, let $\hat{G}_{2}(s), \hat{G}_{3}(s)$ be the solution to

$$
\begin{aligned}
& \hat{G}_{3}(s) v^{2}+\left(1-\hat{G}_{3}(s)\right) v^{3}-c_{2} s=u_{2}^{*} \\
& \hat{G}_{2}(s) v^{2}+\left(1-\hat{G}_{2}(s)\right) v^{3}-c_{3} s=u_{3}^{*}
\end{aligned}
$$

for $s \in[0,0.05]$, and let $\hat{G}_{1}(s), \hat{G}_{2}(s), \hat{G}_{3}(s)$ be the solution to (1) to (3) for $s \in[0.05,0.57]$. The resulting functions $\hat{G}_{1}, \hat{G}_{2}, \hat{G}_{3}$ are referred to as the "pseudo strategies yielding payoffs $u_{1}^{*}, u_{2}^{*}$ and $u_{3}^{*}$. Figure 3 plots the pseudo strategies. As we can see, a pseudo strategy may not be monotone, therefore may not be a mixed strategy. ${ }^{11}$

In the above example, we define the pseudo strategies given that players 2 and 3 are participating at scores in [0,0.05] and all three players are participating at scores in [0.05, 0.57]. However, we generally do not know who are participating beforehand. Therefore, the general definition of pseudo strategies also needs to determine the participating players at each score. This is the key part of the definition. As above, once we know who are participating at a score, the values of pseudo strategies at the score are determined by the indifference conditions for the participating players.

Therefore, we need some notations to represent the indifference conditions for the general case. Define a function $W_{\mathcal{P}}:[0,1]^{|\mathcal{P}|-1} \times[0, \infty)^{|\mathcal{P}|} \rightarrow[0, \infty)$ that takes the form

$$
W_{\mathcal{P}}\left(\mathbf{x}_{-j}, \mathbf{v}\right)=\sum_{k=1}^{|\mathcal{P}|}\left(v^{(k)} \sum_{P_{k-1} \in \mathcal{D}_{k-1}}\left(\prod_{i \in P_{k-1}}\left(1-x_{i}\right) \prod_{i^{\prime} \in(\mathcal{P} \backslash\{j\}) \backslash P_{k-1}} x_{i^{\prime}}\right)\right)
$$

where $j \in \mathcal{P}, \mathbf{x}_{-j}=\left(x_{i}\right)_{i \in \mathcal{P} \backslash\{j\}} \in[0,1]^{|\mathcal{P}|-1}, \mathbf{v} \in[0, \infty)^{|\mathcal{P}|}, v^{(k)}$ is the $k$ th highest element in $\mathbf{v}$, and $\mathcal{D}_{k-1}=\left\{P_{k-1} \mid P_{k-1} \subseteq \mathcal{P} \backslash\{j\}\right.$ and $\left.\left|P_{k-1}\right|=k-1\right\}$. Consider a contest with a set of players $\mathcal{P}$ and prizes $\mathbf{v}=\left(v^{k}\right)_{k \in \mathcal{P}}$. Let $\mathbf{G}_{-j}=\left(G_{k}\right)_{k \in \mathcal{P} \backslash\{j\}}$ be a profile of mixed strategies of the players other than $j$. Suppose these players choose the strategies $\mathbf{G}_{-j}$ and suppose player $j$ chooses score $s$. Then, player $j$ 's expected winnings is $W_{\mathcal{P}}\left(\mathbf{G}_{-j}(s), \mathbf{v}\right)$. Therefore, the indifference condition for

\footnotetext{
${ }^{11}$ Throughout, by monotone, we mean non-decreasing.
} 
player $j \in \mathcal{P}$ is

$$
W_{\mathcal{P}}\left(\mathbf{G}_{-j}(s), \mathbf{v}\right)-C_{j}(s)=u_{j}^{*}
$$

In Example 1, we have $\mathcal{P}=\{1,2,3\}$ and $v^{3}=0$. Then, equations (4) for $j \in \mathcal{P}$ are the same as equations (1) to (3). Since the domain of $W_{\mathcal{P}}$ can be determined from the dimensions of its arguments $\mathbf{G}_{-j}(s)$ and $\mathbf{v}$, we omit the subscript $\mathcal{P}$ if there is no ambiguity.

Let us present the definition of pseudo strategies for the general case. Given an equilibrium, denote the equilibrium payoffs as $u_{1}^{*}, u_{2}^{*}, \ldots, u_{i}^{*}$ for some $i \leq m+1$. We define pseudo strategies $\hat{G}_{1}, \ldots, \hat{G}_{i}$ yielding the payoffs in four steps:

First, we define $\hat{\mathcal{P}}\left(\underline{s}_{i}\right)=\{i-1, i\}$ at a score $\underline{s}_{i}$, which solves $v^{i}-C_{i}\left(\underline{s}_{i}\right)=u_{i}^{*}$. ${ }^{12}$ The equation means that player $i$ receives $u_{i}^{*}$ if he wins prize $v^{i}$ by choosing $\underline{s}_{i}$. We define pseudo strategies of the players in $\hat{\mathcal{P}}\left(\underline{s}_{i}\right)$ for $s \geq \underline{s}_{i}$ until one of the pseudo strategies reaches 1 . More precisely, consider equation system (4) for $\mathcal{P}=\{i-1, i\}$ and $j \in\{i-1, i\}$. Under Assumptions A1 and A2, there exists a score $\bar{s}_{i} \geq \underline{s}_{i}$ such that the system has a unique solution $\left(\hat{G}_{i-1}(s), \hat{G}_{i}(s)\right)$ in $[0,1]^{2}$ for $s \in\left[\underline{s}_{i}, \bar{s}_{i}\right]$, where $\bar{s}_{i}$ satisfies $\hat{G}_{i}\left(\bar{s}_{i}\right)=1 .^{13}$ For $k \notin\{i-1, i\}$, let $\hat{G}_{k}(s)=0$ for $s \in\left[\underline{s}_{i}, \bar{s}_{i}\right]$.

Second, we extend the definitions of $\hat{\mathcal{P}}\left(\underline{s}_{i}\right)$ to higher scores until the payoff of $i-2$, the weast among those stronger than the ones in $\hat{\mathcal{P}}\left(\underline{s}_{i}\right)$, obtains his equilibrium payoff. More precisely, there exists $\underline{s}_{i-2} \in\left[\underline{s}_{i}, \bar{s}_{i}\right]$ such that

$$
W\left(\hat{G}_{i-1}\left(\underline{s}_{i-2}\right), \hat{G}_{i}\left(\underline{s}_{i-2}\right), v^{i-2}, v^{i-1}, v^{i}\right)-C_{i-2}\left(\underline{s}_{i-2}\right)=u_{i-2}^{*}
$$

Note that the above equation includes prizes $\left(v^{k}\right)_{k \in \hat{\mathcal{P}}\left(\underline{s}_{i}\right)}$ and $v^{i-2}$, which is the lowest prize among those higher than $\left(v^{k}\right)_{k \in \hat{\mathcal{P}}\left(\underline{s}_{i}\right)}$. Then, we define $\hat{\mathcal{P}}(s)=\{i-1, i\}$ for $s \in\left(\underline{s}_{i}, \underline{s}_{i-2}\right)$ and $\hat{\mathcal{P}}\left(\underline{s}_{i-2}\right)=$ $\{i-2, i-1, i\}$.

Third, given $\hat{\mathcal{P}}\left(\underline{s}_{i-2}\right)$ we proceed as in the first step and redefine pseudo strategies of the players in $\hat{\mathcal{P}}\left(\underline{s}_{i-2}\right)$ for $s \geq \underline{s}_{i-2}$ until one of two cases arises. In the first case, player $i-3$, the weakest among those stronger than the ones in $\hat{\mathcal{P}}\left(\underline{s}_{i-2}\right)$, obtains his equilibrium payoff. Let $\underline{s}_{i-3}$ denote the score at which $i-3$ obtains his equilibrium payoff. Then, as in the second step, define $\hat{\mathcal{P}}(s)=\{i-2, i-1, i\}$ for $s \in\left(\underline{s}_{i-2}, \underline{s}_{i-3}\right)$ and $\hat{\mathcal{P}}\left(\underline{s}_{i-3}\right)=\hat{\mathcal{P}}\left(\underline{s}_{i-2}\right) \cup\{i-3\}$. In the second case, the pseudo strategy of player $i$, the weakest in $\hat{\mathcal{P}}\left(\underline{s}_{i-2}\right)$, reaches 1 . Let $\bar{s}_{i}$ denote the score at which $i$ 's pseudo strategy reaches 1 . Then, define $\hat{\mathcal{P}}(s)=\{i-2, i-1, i\}$ for $s \in\left(\underline{s}_{i-2}, \bar{s}_{i}\right)$ and $\hat{\mathcal{P}}\left(\bar{s}_{i}\right)=\hat{\mathcal{P}}\left(\underline{s}_{i-2}\right) \backslash\{i\}$. If both cases happen at the same score $s^{\prime}$, then let $\bar{s}_{i}=\underline{s}_{i-3}=s^{\prime}, \hat{\mathcal{P}}(s)=\{i-2, i-1, i\}$ for $s \in\left(\underline{s}_{i-2}, s^{\prime}\right)$, and $\hat{\mathcal{P}}\left(s^{\prime}\right)=\left(\hat{\mathcal{P}}\left(\underline{s}_{i-2}\right) \backslash\{i\}\right) \cup\{i-3\}$.

Fourth, suppose the we extend the definition of $\hat{\mathcal{P}}$ to score $s^{\prime}$, where $s^{\prime}=\bar{s}_{i}, \underline{s}_{i-3}$ or both. After that, given $\hat{\mathcal{P}}\left(s^{\prime}\right)$ we continue as in the third step and extend the pseudo strategies of the players in $\hat{\mathcal{P}}\left(s^{\prime}\right)$ to higher scores until the weakest player among those stronger than the ones in $\hat{\mathcal{P}}\left(s^{\prime}\right)$ obtains his equilibrium payoff, or until the pseudo strategy of the weakest player in $\hat{\mathcal{P}}\left(s^{\prime}\right)$ reaches

\footnotetext{
${ }^{12}$ Note that if $i<m+1$, we are considering fewer players than in the equilibrium, so $\hat{\mathcal{P}}(s)$ defined below may be different from $\mathcal{P}^{*}(s)$ in the equilibrium.

${ }^{13}$ Lemma 6 in Appendix A verifies the existence of the unique solution and other claims in the definition of pseudo strategies.
} 
1. Continuing in this fashion, we can find intervals $\left[\underline{s}_{1}, \bar{s}_{1}\right], \ldots,\left[\underline{s}_{i}, \bar{s}_{i}\right]$ and define $\hat{G}_{j}(s)$ for $s \in\left[\underline{s}_{j}, \bar{s}_{j}\right]$ with $\hat{G}_{j}\left(\underline{s}_{j}\right)=0$ and $\hat{G}_{j}\left(\bar{s}_{j}\right)=1$ for $j=1, \ldots, i$. Moreover, we can verify that under Assumptions $\mathrm{A} 1$ and A2, $\hat{G}_{j}(s) \leq \hat{G}_{j^{\prime}}(s)$ for $j<j^{\prime}$ at any $s \in\left[\underline{s}_{j}, \bar{s}_{j}\right] \cap\left[\underline{s}_{j^{\prime}}, \bar{s}_{j^{\prime}}\right] .{ }^{14}$ We refer to functions $\hat{G}_{1}, \ldots, \hat{G}_{i}$ as the pseudo strategies yielding $u_{1}^{*}, \ldots, u_{i}^{*} ; \hat{\mathcal{P}}(s)$ as the set of participating players at $s$ for the pseudo strategies; and the left hand of (5) as player $i-2$ 's payoff at $\underline{s}_{i-2}$ against pseudo strategies $\hat{G}_{i-1}$ and $\hat{G}_{i}$.

Note that for arbitrary payoffs, e.g. $u_{i}^{*}<u_{i+1}^{*}$, the above procedure may not apply. The two propositions below discuss the relationship between equilibrium strategies and pseudo strategies. These propositions are important to the proof of Theorem 1 as the algorithm in Section 3.2 constructs the equilibrium strategies by examining the pseudo strategies. Assumption A2 of geometric or quadratic prize sequences is important for the propositions.

We say that a continuous function $G$ has a dent over $\left(s^{\prime}, s^{\prime \prime}\right)$ if $\left(s^{\prime}, s^{\prime \prime}\right)$ is the largest interval such that i) $G\left(s^{\prime}\right)=G\left(s^{\prime \prime}\right)$; ii) $G(s) \leq G\left(s^{\prime}\right)$ for $s \in\left(s^{\prime}, s^{\prime \prime}\right)$. For example, $\hat{G}_{3}$ in Figure 2 has a dent over $(0.05,0.34)$.

Proposition 4 (Dent vs Gap) Take any equilibrium with equilibrium payoffs $u_{1}^{*}, u_{2}^{*}, \ldots, u_{n}^{*}$. Then, for any $i \leq m+1$, let $\hat{G}_{1}, \ldots, \hat{G}_{i}$ be the pseudo strategies yielding the payoffs $u_{1}^{*}, \ldots, u_{i}^{*}$. The following two statements are equivalent:

i) player i's equilibrium strategy has a gap $\left(s_{i}^{\prime}, s_{i}^{\prime \prime}\right)$ in its support,

ii) player $i$ 's pseudo strategy has a dent over $\left(s_{i}^{\prime}, s_{i}^{\prime \prime}\right)$.

In Figures 2 and 3 , the dent of $\hat{G}_{3}$ indeed coincides with the gap of $G_{3}^{*}$. Let us use Example 1 to explain the proposition. Suppose $\hat{G}_{3}\left(s^{\prime}\right)>G_{3}^{*}\left(s^{\prime}\right)$ for $s^{\prime}=0.2$, then Proposition 4 is violated. Recall that both $\left(\hat{G}_{1}\left(s^{\prime}\right), \hat{G}_{2}\left(s^{\prime}\right), \hat{G}_{3}\left(s^{\prime}\right)\right)$ and $\left(G_{1}^{*}\left(s^{\prime}\right), G_{2}^{*}\left(s^{\prime}\right), G_{3}^{*}\left(s^{\prime}\right)\right)$ satisfy (1) and (2), and we can verify that $\hat{G}_{3}\left(s^{\prime}\right)>G_{3}^{*}\left(s^{\prime}\right)$ implies $\hat{G}_{1}\left(s^{\prime}\right)<G_{1}^{*}\left(s^{\prime}\right)$ and $\hat{G}_{2}\left(s^{\prime}\right)<G_{2}^{*}\left(s^{\prime}\right)$. This means if player 3's pseudo strategy increases at a score, it requires 1 and 2's pseudo strategies to be lower at this score to maintain 1 and 2's payoffs. Therefore, if players 1 and 2 choose $\hat{G}_{1}$ and $\hat{G}_{2}$ instead of $G_{1}^{*}$ and $G_{2}^{*}$, their scores are more likely to be higher than $s^{\prime}$. Hence, player 3's payoff by choosing $s^{\prime}$ is lower than his equilibrium payoff if others choose $\hat{G}_{1}$ and $\hat{G}_{2}$. This contradicts the definition of $\hat{G}_{1}$ and $\hat{G}_{2}$ as they are supposed to satisfy (3) at $s^{\prime}$ and give player 3 his equilibrium payoff. In the general case, it remains true that if one player's pseudo strategy increases at a score, it requires the other players' pseudo strategies to be lower at this score to maintain the other players' payoffs. We use this property to prove Propositions 4 and 5 in Appendix A. In Figures 2 and 3, the equilibrium strategy $G_{3}^{*}$ is the smallest monotone function that lies on or above $\hat{G}_{3}$. This is because $3=m+1$ in the example. For $i<m+1$, the equilibrium strategy $G_{i}^{*}$ may not be the smallest monotone function that lies on or above the pseudo strategy $\hat{G}_{i}$ in Proposition $4 .^{15}$

\footnotetext{
${ }^{14}$ The proof is the same as Step I in the proof of Proposition 1.

${ }^{15}$ To see why, consider Example 1 in Section 3.2. Notice that $\bar{G}_{1}^{2}$ and $\bar{G}_{2}^{2}$ in Step 1.2 are the pseudo strategies in the contest with the two most efficient players 1 and 2 . However, $G_{2}^{*}$ is not the smallest monotone function that lies on or above $\bar{G}_{2}^{2}$.
} 
Proposition 5 Take any equilibrium and let $u_{1}^{*}, u_{2}^{*} \ldots, u_{n}^{*}$ denote the equilibrium payoffs. For any $i \leq m+1$, suppose $\hat{G}_{1}, \ldots, \hat{G}_{i}$ are the pseudo strategies yielding the equilibrium payoffs $u_{1}^{*}, \ldots, u_{i}^{*}$, and let $\hat{S}_{i}=\left\{s \mid \hat{G}_{i}\right.$ is increasing at $s$ and $s$ is not contained in any dent of $\left.\hat{G}_{i}\right\}$. Then, in the contest of prizes $v^{1}, \ldots, v^{i+1}$ and players $1, \ldots, i+1$, player $i+1$ 's equilibrium payoff is the payoff corresponding to his best responses in $\hat{S}_{i}$ against others' pseudo strategies $\hat{G}_{1}, \ldots, \hat{G}_{i}$.

Let us explain the proposition in the context of Example 1. Recall that players 1 and 2's equilibrium payoffs in the example are $u_{1}^{*}=1.72$ and $u_{2}^{*}=0.58$. The pseudo strategies yielding the payoffs are $\hat{G}_{1}(s)=1.67 s+0.24$ and $\hat{G}_{2}(s)=2 s-0.14$ for $s \in[0.18,0.57]$. Because $\hat{G}_{2}$ has no dent, $\hat{S}_{2}=[0.18,0.57]$. We can verify that $\bar{s}_{3}^{*}=0.57$ is player 3 's best response in $\hat{S}_{2}=[0.18,0.57]$ against $\hat{G}_{1}$ and $\hat{G}_{2}$ and it gives him a payoff of $u_{3}^{*}$. Why do the other scores give player 3 lower payoffs? Consider $s=0.4$ for example. Recall that we exclude player 3 from the contest when we define the pseudo strategies. Similar to the discussion after Proposition 4, we have $\hat{G}_{1}(s)<G_{1}^{*}(s)$ and $\hat{G}_{2}(s)<G_{2}^{*}(s)$. Therefore, the probability of player 1's score being below $s$ decreases from $G_{1}^{*}(s)$ to $\hat{G}_{1}(s)$. As a result, facing $\hat{G}_{1}(s)$ and $\hat{G}_{2}(s)$, player 3 's payoff by choosing $s$ is lower than his equilibrium payoff. Section 3.2 introduces an algorithm using this proposition to determine $i+1$ 's equilibrium payoff. Note that in Proposition 5, it is important to exclude the scores outside of $\hat{S}_{i}$ when searching for $i+1$ 's best responses. This is because if we include the scores outside of $\hat{S}_{i}$, the algorithm would not construct the equilibrium. ${ }^{16}$

\subsection{Algorithm}

The existing methods cannot construct the equilibrium because i) there could be gaps in the supports of mixed strategies, and ii) the equilibrium payoffs cannot be derived without examining the strategies. As a result, we propose a new algorithm below. To distinguish from the equilibrium strategy $G_{i}^{*}$ and payoff $u_{i}^{*}$, we use $\hat{G}_{i}^{*}$ and $\hat{u}_{i}^{*}$ to represent the outcome of the algorithm.

In the remainder of this section, we first explain the algorithm in the context of Example 1, then present the algorithm in the general case.

Example 1 (continued) Recall that, in Example 1, the highest score in the support of $G_{1}^{*}$ is $\bar{s}_{1}^{*}=0.57$. The algorithm below starts with a guess of the highest score in the support of $G_{1}^{*}$. Step 1.2 first defines payoffs $u_{1}, u_{2}$ for the top two players 1 and 2, then derives their pseudo strategies yielding the payoffs. Step 1.3 first determines a payoff $u_{3}$ for the next strongest player - player 3 - using his best response against the pseudo strategies derived in Step 1.2, then derives the pseudo strategies yielding $u_{1}, u_{2}, u_{3}$. Step 2 shifts all the pseudo strategies, and the resulting payoffs are equal to the equilibrium payoffs. After Step 2, the pseudo strategies are continuous and their values are between 0 and 1, but they may not be monotone. Step 3 fixes the non-monotonicity.

Step 1.1. Take an arbitrary guess of the highest score in the support of $G_{1}^{*}$. Here let the guess be $\bar{s}=1$. Proposition 7 below shows that the final outcome of the algorithm does not depend on the initial guess.

\footnotetext{
${ }^{16}$ For example, see Step 1.3 in Example 1 in Section 3.2.
} 
Step 1.2. Consider a contest with only players 1 and 2 and prizes $v^{1}$ and $v^{2}$. Let $\bar{s}_{1}=\bar{s}_{2}=\bar{s}$, which means the highest scores in the supports of 1 and 2's mixed strategies are the same, and equal to $\bar{s}$. Then, if player 1 chooses $\bar{s}$, he would win the first prize $v^{1}$. The resulting payoff is $u_{1}=v^{1}-c_{1} \bar{s}=0$. Similarly, if 2 chooses $\bar{s}$, his payoff is $u_{2}=v^{1}-c_{2} \bar{s}=-2$. If the guess $\bar{s}$ were correct, the payoffs $u_{1}$ and $u_{2}$ defined above would be the equilibrium payoffs. Since $\bar{s}=1$ is not a correct guess, the resulting payoffs could be negative, but the payoffs are revised to the equilibrium ones in Step 2 below.

Having defined payoffs $u_{1}$ and $u_{2}$, we can find the pseudo strategies $\bar{G}_{1}^{2}$ and $\bar{G}_{2}^{2}$ in the two-player contest yielding these payoffs. The superscripts represent the step in which the pseudo strategies are defined. In particular, consider the following equations

$$
\begin{aligned}
\bar{G}_{2}^{2}(s) v^{1}+\left(1-\bar{G}_{2}^{2}(s)\right) v^{2}-c_{1} s & =u_{1} \\
\bar{G}_{1}^{2}(s) v^{1}+\left(1-\bar{G}_{1}^{2}(s)\right) v^{2}-c_{2} s & =u_{2}
\end{aligned}
$$

where the first equation ensures that each score gives player 1 the same payoff $u_{1}$, and the second equation is analogous. The solution is $\bar{G}_{1}^{2}(s)=2 s-1$ and $\bar{G}_{2}^{2}(s)=(4 s-1) / 3$. Note that both $\bar{G}_{1}^{2}$ and $\bar{G}_{2}^{2}$ have the same support $[1 / 2,1]$.

Step 1.3. Consider the three-player contest. Suppose other players choose strategies $\bar{G}_{1}^{2}$ and $\bar{G}_{2}^{2}$. Player 3 's best response in $\bar{G}_{2}^{2}$ 's support $[1 / 2,1]$ is $\bar{s}_{3}=1$, and his payoff associated with the best response is $u_{3}=-3$. In this particular example, the best response happens to be the highest score in the support, which may not true in other examples. Note that we need to restrict the search of best response to the support $[1 / 2,1]$ of $\hat{G}_{2}^{2}$. This is because a score outside $[1 / 2,1]$, e.g. $s=0$, can result in a higher payoff for player 3 . If we included the scores outside of $[1 / 2,1]$, we would find a different best response in this step, with which we would not construct the equilibrium.

Given payoffs $u_{1}, u_{2}, u_{3}$ defined above, we can derive pseudo strategies $\bar{G}_{1}^{3}, \bar{G}_{2}^{3}, \bar{G}_{3}^{3}$ yielding $u_{1}, u_{2}, u_{3}$. In particular, let $\bar{G}_{2}^{3}(s)$ and $\bar{G}_{3}^{3}(s)$ be the solution to the system

$$
\begin{aligned}
\bar{G}_{3}^{3}(s) v^{2}-c_{2} s & =u_{2} \\
\bar{G}_{2}^{3}(s) v^{2}-c_{3} s & =u_{3}
\end{aligned}
$$

so $\bar{G}_{2}^{3}(s)$ and $\bar{G}_{3}^{3}(s)$ are defined over $[0.43,+\infty)$, where $\bar{G}_{2}^{3}(0.43)=0$. Then, let $\underline{s}_{1}$ be the smallest score at which

$$
\bar{G}_{2}^{3}(s) \bar{G}_{3}^{3}(s) v^{1}+\left[\bar{G}_{2}^{3}(s)\left(1-\bar{G}_{3}^{3}(s)\right)+\left(1-\bar{G}_{2}^{3}(s)\right) \bar{G}_{3}^{3}(s)\right] v^{2}-c_{1} s=u_{1}
$$

The equation means that given $\bar{G}_{2}^{3}(s)$ and $\bar{G}_{3}^{3}(s)$, player 1 's payoff by choosing s equals $u_{1}$. For each $s \in\left[\underline{s}_{1}, \bar{s}\right]$, update the definitions of $\bar{G}_{1}^{3}, \bar{G}_{2}^{3}, \bar{G}_{3}^{3}$ to be the solution to the 
following system:

$$
\begin{aligned}
\bar{G}_{2}^{3}(s) \bar{G}_{3}^{3}(s) v^{1}+\left[\bar{G}_{2}^{3}(s)\left(1-\bar{G}_{3}^{3}(s)\right)+\left(1-\bar{G}_{2}^{3}(s)\right) \bar{G}_{3}^{3}(s)\right] v^{2}-c_{1} s & =u_{1} \\
\bar{G}_{1}^{3}(s) \bar{G}_{3}^{3}(s) v^{1}+\left[\bar{G}_{1}^{3}(s)\left(1-\bar{G}_{3}^{3}(s)\right)+\left(1-\bar{G}_{1}^{3}(s)\right) \bar{G}_{3}^{3}(s)\right] v^{2}-c_{2} s & =u_{2} \\
\bar{G}_{1}^{3}(s) \bar{G}_{2}^{3}(s) v^{1}+\left[\bar{G}_{1}^{3}(s)\left(1-\bar{G}_{2}^{3}(s)\right)+\left(1-\bar{G}_{1}^{3}(s)\right) \bar{G}_{2}^{3}(s)\right] v^{2}-c_{3} s & =u_{3}
\end{aligned}
$$

Step 2. Shift $\bar{G}_{1}^{3}, \bar{G}_{2}^{3}, \bar{G}_{3}^{3}$ horizontally, so that the resulting pseudo strategies $\hat{G}_{1}^{3}, \hat{G}_{2}^{3}, \hat{G}_{3}^{3}$ satisfy $\hat{G}_{2}^{3}(0)=0$. It turns out that they shift 0.43 to the left. Figure 3 depicts the pseudo strategies after the shift, where the superscripts are omitted. The shift also affects the payoffs, and the payoffs after the shift is $\hat{u}_{i}^{*}=u_{i}+0.43 c_{i}$ for $i=1,2,3$. It turns out they are equal to the equilibrium payoffs.

Step 3. Notice that $\hat{G}_{3}^{3}$ is not monotone, so it cannot be a mixed strategy. This step fixes the nonmonotonicity. Define $\hat{G}_{3}^{*}$ to be the smallest monotone function $G$ that satisfies $G(s) \geq$ $\hat{G}_{3}^{3}(s)$ for all $s \geq 0$. Actually, $\hat{G}_{3}^{*}$ as defined above is the equilibrium strategy $G_{3}^{*}$ in Figure 2. There is a gap $(0.05,0.34)$ in the support of $\hat{G}_{3}^{*}$, so only 1 and 2 mix concurrently over the interval. For $s \in(0.05,0.34)$, let $\hat{G}_{1}^{*}(s)$ and $\hat{G}_{2}^{*}(s)$ be the solution to the system

$$
\begin{aligned}
& \hat{G}_{2}^{*}(s) \hat{G}_{3}^{*}(s) v^{1}+\left[\hat{G}_{2}^{*}(s)\left(1-\hat{G}_{3}^{*}(s)\right)+\left(1-\hat{G}_{2}^{*}(s)\right) \hat{G}_{3}^{*}(s)\right] v^{2}-c_{1} s=\hat{u}_{1}^{*} \\
& \hat{G}_{1}^{*}(s) \hat{G}_{3}^{*}(s) v^{1}+\left[\hat{G}_{1}^{*}(s)\left(1-\hat{G}_{3}^{*}(s)\right)+\left(1-\hat{G}_{1}^{*}(s)\right) \hat{G}_{3}^{*}(s)\right] v^{2}-c_{2} s=\hat{u}_{2}^{*}
\end{aligned}
$$

Notice that the equations are the same as (6) and (7) except that $\hat{G}_{3}^{*}(s)$ is known and remains constant.

For $s \notin(0.05,0.34)$, let $\hat{G}_{i}^{*}(s)=\hat{G}_{i}^{3}(s)$. As a result, we have obtained three mixed strategies $\hat{G}_{1}^{*}, \hat{G}_{2}^{*}$ and $\hat{G}_{3}^{*}$, which are the same as the equilibrium strategies in Figure 2.

There are two major challenges to construct the equilibrium: determining the equilibrium payoffs and identifying the gap. We overcome the first challenge in Step 1.3 and Step 2. In particular, Step 1.3 defines payoff $u_{3}$ by examining player 3's best response against the strategies in a contest with only players 1 and 2 . Then, Step 2 shifts the pseudo strategies, which revises the payoff $u_{i}$ to the equilibrium one $u_{i}^{*}$. We overcome the second challenge in Step 3. In particular, we find the gap by comparing pseudo strategy $\hat{G}_{3}^{3}$ with the smallest monotone function on or above it. We can determine equilibrium payoffs by the shift in Step 2 because of linear cost functions, which is discussed in more details in Proposition 8. Section 4 discusses how to modify the algorithm for nonlinear cost functions.

Now let us discuss the algorithm in the general case. Compared to the above example, there are two complications. First, we need to define payoffs and pseudo strategies for more players, that is, players $4, \ldots, m+1$ besides 1,2 and 3 . Therefore, after Step 1.3 we continue to define a payoff for the next strongest player, 4 , and derive the pseudo strategies for the top four players. Then, we proceed in the same fashion for players $5,6, \ldots, m+1$. Second, we may need to fix the monotonicity for multiple pseudo strategies. To overcome this complication, starting from the weakest player's, 
Step 3 fixes one pseudo strategy at a time until all of them are monotone. Figure 4 contains a schematic representation of the algorithm. The algorithm may involve negative scores, so we extend the cost functions such that $C_{i}(s)=c_{i} s$ for any $s \in \mathbb{R}$. We describe below the algorithm for the general case.

Step 1.1 To start the algorithm, pick any $\bar{s}>0$.

Step 1.2 Suppose only players 1 and 2 compete for $v^{1}$ and $v^{2}$. Define $u_{i}=v^{1}-c_{i} \bar{s}_{2}$ for $i=1,2$. Given $u_{1}$ and $u_{2}$, let $\bar{G}_{1}^{2}, \bar{G}_{2}^{2}$ be the pseudo strategies yielding $u_{1}$ and $u_{2}$. Let $\left[\underline{s}_{1}, \bar{s}_{2}\right]$ be the scores where $\bar{G}_{1}^{2}, \bar{G}_{2}^{2}$ are defined, so $\bar{G}_{1}^{2}\left(\underline{s}_{1}\right)=0$. The superscripts indicate the step that the pseudo strategies are defined.

Repeat the following step for $i=3, \ldots, m+1$.

Step 1.i Suppose Step 1. $(i-1)$ has defined pseudo strategies $\bar{G}_{1}^{i-1}, \ldots, \bar{G}_{i-1}^{i-1}$ yielding $u_{1}, \ldots, u_{i-1}$. Suppose $\bar{G}_{i-1}^{i-1}$ is defined over $\left[\underline{s}_{i-2}, \bar{s}_{i-1}\right]$ with $\bar{G}_{i-1}^{i-1}\left(\underline{s}_{i-2}\right)=0$. Suppose players $1, \ldots, i$ compete for prize $v^{1}, \ldots, v^{i}$. Let $u_{i}$ be player $i$ 's payoff corresponding to his best response in $\left[\underline{s}_{i-2}, \bar{s}_{i-1}\right]$ against others with pseudo strategies $\bar{G}_{1}^{i-1}, \ldots, \bar{G}_{i-1}^{i-1}$.

Let $\bar{G}_{1}^{i}, \ldots, \bar{G}_{i}^{i}$ be the pseudo strategies yielding $u_{1}, \ldots, u_{i}$. Note that $\bar{G}_{i}^{i}$ is now defined over $\left[\underline{s}_{i-1}, \bar{s}_{i}\right]$, where $\bar{G}_{i-1}^{i}\left(\underline{s}_{i-1}\right)=0$.

Step 2 Define $\hat{G}_{i}^{m+1}(s)=\bar{G}_{i}^{m+1}\left(s+\underline{s}_{m}\right)$ for $i=1, \ldots, m+1$. If $\underline{s}_{m}>0$, each pseudo strategy is shifted horizontally $\underline{s}_{m}$ to the left to be $\hat{G}_{i}^{m+1}$. After the shift, the payoffs associated with the pseudo strategies become $\hat{u}_{i}^{*}=u_{i}+C_{i}\left(\underline{s}_{m}\right)$ for $i=1, \ldots, m+1$. In particular, $\hat{u}_{m+1}^{*}=0$. Note that $\hat{G}_{i}^{m+1}$ may be decreasing at some scores and so it may not be a mixed strategy.

Next, we fix the non-monotonicity of the pseudo strategies $\hat{G}_{1}^{m+1}, \ldots, \hat{G}_{m+1}^{m+1}$. Repeat the following step for $i=m+1, m, \ldots, 3$.

Step 3.i Suppose the pseudo strategies before this step are $\hat{G}_{1}^{i}, \ldots, \hat{G}_{i}^{i}$, and $\hat{G}_{i+1}^{*}, \ldots, \hat{G}_{m+1}^{*}$ if $i<$ $m+1$. This step defines $G_{i}^{*}$ and updates $G_{1}^{i}, \ldots, G_{i-1}^{i}$ while keeping $G_{i+1}^{*}, \ldots, G_{m+1}^{*}$ the same. Similar to Proposition $3, \hat{G}_{i}^{i}$ is differentiable, and $\hat{G}_{i}^{i \prime}(s) \geq \hat{G}_{j}^{i \prime}(s)$ if $j>i$. Therefore, if $\hat{G}_{i}^{i}$ is monotone, so are $\hat{G}_{1}^{i}, \ldots, \hat{G}_{i-1}^{i}$. Then, rename $\hat{G}_{j}^{i}$ to $\hat{G}_{j}^{*}$ for all $j \leq i$ and move to Step 4. If $\hat{G}_{i}^{i}$ is not monotone, let $\hat{G}_{i}^{*}$ be the smallest monotone function that lies on or above $\hat{G}_{i}^{i}$.

Find all the dents of $\hat{G}_{i}^{i}$, and it can be verified that $\hat{G}_{i}^{i}$ has a finite number of dents. ${ }^{17}$ Pick any $\operatorname{dent}\left(s^{\prime}, s^{\prime \prime}\right)$. For any $s \in\left(s^{\prime}, s^{\prime \prime}\right)$, let $G_{i}(s)=\hat{G}_{i}^{*}(s)$ for $j \geq i$ and substitute them into system (4) for $j \in \hat{\mathcal{P}}^{i}(s) \backslash\{i, \ldots, m+1\}$, where $\hat{\mathcal{P}}^{i}(s)$ is the set of participating players at $s^{\prime}$ for the pseudo strategies $\hat{G}_{1}^{i}, \ldots, \hat{G}_{i}^{i}, \hat{G}_{i+1}^{*}, \ldots, \hat{G}_{m+1}^{*}$. The resulting system has $\left|\hat{\mathcal{P}}^{i}\left(s^{\prime}\right) \backslash\{i, \ldots, m+1\}\right|$ equations with the same number of unknowns $\left(G_{j}(s)\right)_{j \in \hat{\mathcal{P}}^{i}\left(s^{\prime}\right) \backslash\{i, \ldots, m+1\}} \cdot$

\footnotetext{
${ }^{17}$ The proof of Proposition 6 in Appendix B verifies that there are at most a finite number of dents.
} 
If $\hat{\mathcal{P}}^{i}\left(s^{\prime}\right) \backslash\{i, \ldots, m+1\}=\hat{\mathcal{P}}^{i}\left(s^{\prime \prime}\right) \backslash\{i, \ldots, m+1\}$, the resulting system has a unique solution $\left(G_{j}(s)\right)_{j \in \hat{\mathcal{P}}^{i}\left(s^{\prime}\right) \backslash\{i, \ldots, m+1\}}$ in $[0,1]^{\mid \hat{\mathcal{P}}^{i}\left(s^{\prime}\right) \backslash\{i, \ldots, m+1\}}$ for $s \in\left(s^{\prime}, s^{\prime \prime}\right){ }^{18}$ Then, for $s \in\left(s^{\prime}, s^{\prime \prime}\right)$, define $\left(\hat{G}_{j}^{i-1}(s)\right)_{\hat{\mathcal{P}}^{i}\left(s^{\prime}\right) \backslash\{i, \ldots, m+1\}}$ as the solution. If $\hat{\mathcal{P}}^{i}\left(s^{\prime}\right) \backslash\{i, \ldots, m+1\} \varsubsetneqq$ $\hat{\mathcal{P}}^{i}\left(s^{\prime \prime}\right) \backslash\{i, \ldots, m+1\}$, there exists $\underline{s}_{l^{\prime}}^{\prime} \in\left(s^{\prime}, s^{\prime \prime}\right)$ such that the resulting system has a unique solution $\left(G_{j}(s)\right)_{j \in \hat{\mathcal{P}}^{i}\left(s^{\prime}\right) \backslash\{i, \ldots, m+1\}}$ for $s \in\left[s^{\prime}, \underline{s}_{l^{\prime}}^{\prime}\right]$ and a player $l^{\prime} \in\left(\hat{\mathcal{P}}^{i}\left(s^{\prime \prime}\right) \backslash \hat{\mathcal{P}}^{i}\left(s^{\prime}\right)\right) \backslash\{i, \ldots, m+$ $1\}$ has his payoff reaches $\hat{u}_{l^{\prime}}^{*}$. That is,

$$
W_{\hat{\mathcal{P}}^{i}\left(s^{\prime}\right) \cup\left\{l^{\prime}\right\}}\left(\left(\hat{G}_{k}\left(\underline{s}_{l^{\prime}}^{\prime}\right)\right)_{k \in \hat{\mathcal{P}}^{i}\left(\underline{s}_{l^{\prime}}^{\prime}\right)},\left(v^{k}\right)_{k \in \hat{\mathcal{P}}^{i}\left(\underline{s}_{l^{\prime}}^{\prime}\right) \cup\left\{l^{\prime}\right\}}\right)-C_{l^{\prime}}\left(\underline{s}_{l^{\prime}}^{\prime}\right)=\hat{u}_{l^{\prime}}^{*}
$$

Then for $s \in\left[s^{\prime}, \underline{s}_{l^{\prime}}^{\prime}\right]$, define $\left(\hat{G}_{j}^{i-1}(s)\right)_{\hat{\mathcal{P}}^{i}\left(s^{\prime}\right) \backslash\{i, \ldots, m+1\}}$ as the solution. After that, let $\hat{\mathcal{P}}^{i}\left(\underline{s}_{l^{\prime}}^{\prime}\right)=\hat{\mathcal{P}}^{i}\left(s^{\prime}\right) \cup\left\{l^{\prime}\right\}$ and we can extend $\left(\hat{G}_{j}^{i-1}(s)\right)_{j \in \hat{\mathcal{P}}^{i}\left(\underline{s}_{l^{\prime}}^{\prime}\right) \backslash\{i, \ldots, m+1\}}$ to higher scores until the pseudo strategy of another player outside of $\hat{\mathcal{P}}^{i}\left(\underline{s}_{l^{\prime}}^{\prime}\right)$ reaches 1 , and continue in the same fashion until $\left(\hat{G}_{j}^{i-1}(s)\right)_{j \in \hat{\mathcal{P}}^{i}\left(s^{\prime \prime}\right) \backslash\{i, \ldots, m+1\}}$ is defined over the interval $\left(s^{\prime}, s^{\prime \prime}\right)$. Note that the possibility that $\hat{\mathcal{P}}^{i}\left(s^{\prime \prime}\right) \backslash\{i, \ldots, m+1\} \varsubsetneqq \hat{\mathcal{P}}^{i}\left(s^{\prime}\right) \backslash\{i, \ldots, m+1\}$ is excluded by the definition of pseudo strategies. ${ }^{19}$

Similarly, we can define $\left(\hat{G}_{j}^{i-1}(s)\right)$ for all the dents, and let $\hat{G}_{j}^{i-1}(s)=\hat{G}_{j}^{i}(s)$ outside the dents. We call $\hat{G}_{1}^{i-1}, \ldots, \hat{G}_{i-1}^{i-1}$ defined in this step as the "pseudo strategies after fixing $\hat{G}_{i}^{i}$ 's non-monotonicity".

Step 3.2 It can be verified that $\hat{G}_{1}^{2}, \hat{G}_{2}^{2}$ after fixing $\hat{G}_{3}^{3}$ are both monotone. Rename $\hat{G}_{i}^{2}$ to $\hat{G}_{i}^{*}$ for $i \leq 2$ and move to Step 4 .

Step 4 So far, we have defined $\hat{G}_{i}^{*}$ for $i=1, \ldots, m+1$. Let $\hat{G}_{i}^{*}(s)=1$ for $i=m+2, \ldots, n$ and for all $s \geq 0$. Then, $\hat{G}_{i}^{*}$ for $i=1, \ldots, n$ have been defined over $\left[0, \bar{s}-\underline{s}_{m}\right]$. The algorithm ends.

The proposition below implies that the construction ends in finite steps, so the algorithm outcome $\hat{G}_{i}^{*}$ is well defined.

Proposition 6 (Finiteness) The algorithm ends in a finite number of steps.

If the algorithm for Example 1 starts with a different value, it would end up with the same strategies. To see why, suppose the algorithm starts with $\bar{s}=2$ instead of 1 . The pseudo strategies $\bar{G}_{1}^{3}, \bar{G}_{2}^{3}, \bar{G}_{3}^{3}$ after Step 1.3 are the same as before except that they are shifted 1 unit to the right. This is because the marginal costs are constant. As a result, after the shift in Step 2, we obtain exactly the same pseudo strategies as in Figure 2. The above observation is generalized in the following proposition, which relies on the assumption of linear costs.

Proposition 7 (Determinateness) The algorithm constructs a unique profile of strategies, and the strategies are independent of the initial value $\bar{s}$.

\footnotetext{
${ }^{18}$ The proof for the solution existence and uniqueness follows a similar argument in Lemma 6 and is omitted.

${ }^{19}$ To see why, suppose $\hat{\mathcal{P}}^{i}\left(s^{\prime \prime} \backslash\{i, \ldots, m+1\}\right) \varsubsetneqq \hat{\mathcal{P}}^{i}\left(s^{\prime}\right) \backslash\{i, \ldots, m+1\}$, then there is a player $j \in \hat{\mathcal{P}}^{i}\left(s^{\prime}\right) \backslash\{i, \ldots, m+1\}$ whose pseudo strategy $\hat{G}_{j}^{i}$ reaches 1 at a score in $\left(s^{\prime}, s^{\prime \prime}\right)$. This is impossible because $\hat{G}_{j}^{i}(s) \leq \hat{G}_{i}^{i}(s)<1$ for $s \in\left(s^{\prime}, s^{\prime \prime}\right)$, where the first inequality is a property derived from the definition of pseudo strategies.
} 
Step 1.2

Pick any $\bar{s}>0 \rightarrow u_{1}, u_{2} \rightarrow \bar{G}_{1}^{2}, \bar{G}_{2}^{2}$ yielding $u_{1}, u_{2}$

replace $i$ with $i+1$

$$
\downarrow i=3
$$

Step 1.i

Find $i$ 's best response $\bar{s}_{i}$ against $\bar{G}_{1}^{i-1}, \ldots, \bar{G}_{i-1}^{i-1} \rightarrow u_{i}$

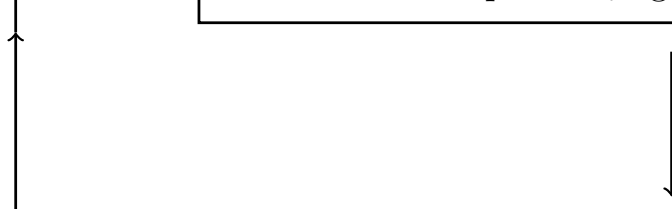

$i \leq m$

$\bar{G}_{1}^{i}, \ldots, \bar{G}_{i}^{i}$ yielding $u_{1}, \ldots, u_{i}$

$$
\downarrow i=m+1
$$

Step 2

Define $\hat{G}_{1}^{m+1}, \ldots, \hat{G}_{m+1}^{m+1}$ by shifting $\bar{G}_{1}^{m+1}, \ldots, \bar{G}_{m+1}^{m+1}$

(1)

Step $3 . i$

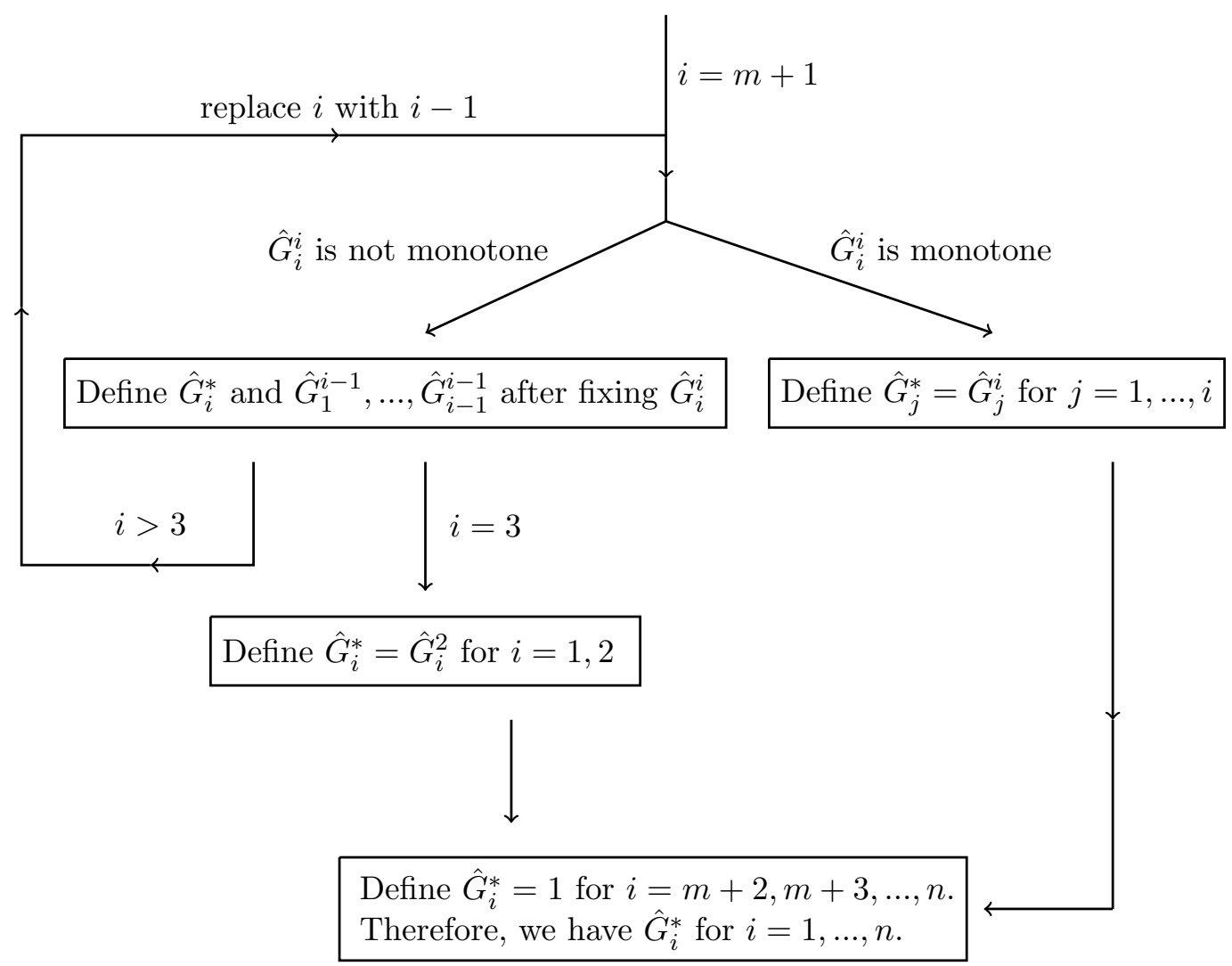

Figure 4: Algorithm 


\subsection{Proof of Unique Equilibrium}

The main objective of this section is to explain how the equilibrium properties and algorithm properties can be used to prove the equilibrium uniqueness.

Consider Example 1 first. Why is the equilibrium unique in the example? Let us explain in two steps. First, given any equilibrium, the algorithm can construct the equilibrium if it starts with a properly chosen $\bar{s}$. We already demonstrated that the algorithm constructs the equilibrium in Figure 2. Let us see why this is true in general. Suppose the algorithm starts with the correct guess $\bar{s}=0.57$, the highest score in the equilibrium. Then, payoffs $u_{1}$ and $u_{2}$ defined in Step 1.2 are exactly the equilibrium payoffs. In Step 1.3, player 3's best response identifies the payoff $u_{3}$, which is exactly his equilibrium payoff. Moreover, by the end of Step 1.3, the algorithm constructs exactly the same pseudo strategies in Figure 3. As a result, Step 2 does not shift the pseudo strategies, and Step 3 modifies the pseudo strategies to the equilibrium ones. Hence, the algorithm indeed constructs the above equilibrium if it starts with $\bar{s}=0.57$.

Second, Proposition 7 implies that if the algorithm starts with a different value, it ends up with exactly the same strategies. Hence, the two steps imply that we must have a unique equilibrium, and it must be the one constructed by the algorithm.

In what follows, we generalize the above argument to prove the uniqueness of equilibrium. In particular, Proposition 8 below generalizes the first step and shows that every equilibrium can be constructed by the algorithm. Note that Proposition 7 already provides a general argument for the second step.

Proposition 8 Given any equilibrium, let $\bar{s}_{1}^{*}$ be the supremum score in the support of player 1 's mixed strategy. If the algorithm starts with $\bar{s}=\bar{s}_{1}^{*}$, it constructs the strategies of the equilibrium.

Proof. We use the equilibrium properties in Section 3.1 to prove the proposition. In particular, Proposition 2 implies that all players choose scores below $\bar{s}_{1}^{*}$ with probability 1 . Therefore, by choosing $\bar{s}_{1}^{*}$, player 1 wins prize $v^{1}$ with probability 1 , hence $u_{1}^{*}=v^{1}-c_{1} \bar{s}_{1}^{*}$. Similarly, $u_{2}^{*}=v^{1}-c_{2} \bar{s}_{1}^{*}$. If the algorithm starts with $\bar{s}=\bar{s}_{1}^{*}$, the payoffs constructed in Step 1.2 are $u_{1}=v^{1}-c_{1} \bar{s}_{1}^{*}=u_{1}^{*}$ and $u_{2}=v^{1}-c_{2} \bar{s}_{1}^{*}=u_{2}^{*}$. Therefore, Step 1.2 constructs pseudo strategies yielding the equilibrium payoffs $u_{1}^{*}$ and $u_{2}^{*}$.

As a result, $u_{3}$ defined in Step 1.3 is player 3's payoff associated with his best response to the pseudo strategies yielding the equilibrium payoffs $u_{1}^{*}$ and $u_{2}^{*}$. Therefore, Proposition 5 implies that $u_{3}$ is exactly the equilibrium payoff of player 3. Then Step 1.3 constructs pseudo strategies yielding the equilibrium payoffs $u_{1}^{*}, u_{2}^{*}$ and $u_{3}^{*}$. By the same argument, Step $1 . i$ defines $u_{i}=u_{i}^{*}$ and constructs pseudo strategies yielding the equilibrium payoffs $u_{1}^{*}, \ldots, u_{i}^{*}$.

By the end of Step 1. $(m+1)$, we have found equilibrium payoffs $u_{1}^{*}, \ldots, u_{m+1}^{*}$ and pseudo strategies yielding these equilibrium payoffs. By definition of pseudo strategies, Step 1. $(m+1)$ defines $\underline{s}_{m}$ such that $v^{m+1}-c_{m+1} \underline{s}_{m}=u_{m+1}^{*}$. Since $u_{m+1}^{*}=0$ and $v^{m+1}=0$, we have $\underline{s}_{m}=0$. As a result, Step 2 does not need to shift the pseudo strategies. Since the algorithm does not change the payoffs after Step 2, the algorithm constructs the equilibrium payoffs. Now we prove that the algorithm constructs the equilibrium strategies. 
First, suppose that there is no gap in the support of player $m+1$ 's equilibrium strategy. Then, Proposition 1 implies that there is no gap in the support of any player's equilibrium strategy. By their definition, the pseudo strategies yielding $u_{1}^{*}, \ldots, u_{m+1}^{*}$ are exactly the equilibrium strategies. Therefore, all the pseudo strategies are monotone. Then, Proposition 4 implies that player $m+1$ 's pseudo strategy has no dent in its support, that is, $\hat{G}_{m+1}^{m+1}$ is monotone. Note the algorithm moves to Step 4 if pseudo strategy $\hat{G}_{m+1}^{m+1}$ is monotone, so the algorithm constructs the equilibrium strategies.

Second, suppose that there is a gap $\left(s^{\prime}, s^{\prime \prime}\right)$ in the support of player $m+1$ 's equilibrium strategy, so $G_{m+1}^{*}(s)=G_{m+1}^{*}\left(s^{\prime}\right)$ for $s \in\left[s^{\prime}, s^{\prime \prime}\right]$. Consider the pseudo strategies yielding $u_{1}^{*}, \ldots, u_{m+1}^{*}$. Proposition 4 implies that player $m+1$ 's pseudo strategy $\hat{G}_{m+1}^{m+1}$ has a dent $\left(s^{\prime}, s^{\prime \prime}\right)$. Step 3. $(m+1)$ defines $\hat{G}_{i}^{*}(s)=\hat{G}_{i}\left(s^{\prime}\right)$ for $s \in\left[s^{\prime}, s^{\prime \prime}\right]$, so $\hat{G}_{i}^{*}(s)=G_{i}^{*}(s)$ for $s \in\left[s^{\prime}, s^{\prime \prime}\right]$. Similarly, $\hat{G}_{i}^{*}(s)=G_{i}^{*}(s)$ for $s$ in any gap in the support of $G_{m+1}^{*}$. For $s$ outside the gaps in the support of $G_{m+1}^{*}$, Proposition 1 implies that no player has a gap containing $s$. Moreover, Lemma 8 implies that each player's equilibrium strategy is absolutely continuous, and it is differentiable except at a finite number of scores. Therefore, the definition of pseudo strategies implies $\hat{G}_{m+1}^{m+1}(s)=G_{m+1}^{*}(s)$. Notice that Step 3. $(m+1)$ defines $\hat{G}_{m+1}^{*}(s)=\hat{G}_{m+1}(s)$ for $s$ outside the dents of $\hat{G}_{m+1}$, so the strategy $\hat{G}_{m+1}^{*}$ constructed in this step is exactly player $m+1$ 's equilibrium strategy $G_{m+1}^{*}$.

Analogously, Propositions 1 and 4 imply that the strategy $\hat{G}_{i}^{*}$ constructed in Step $3 . i$ is the equilibrium strategy $G_{i}^{*}$ for player $i<m+1$. By the end of Step 3.3, the algorithm has fixed the monotonicity of the pseudo strategies of $3, \ldots, m+1$. Since there is no aggregate gap in the equilibrium, there must be no gaps in the equilibrium strategies of 1 and 2, therefore their pseudo strategies after Step 3.3 must be monotone and equal to their equilibrium strategies.

Hence, by the end of Step 3.3, the algorithm constructs the equilibrium strategies for player $1, \ldots, m+1$. Finally, Step 4 specifies the equilibrium strategies for the inactive players $m+2, \ldots, n$, and completes the construction.

Proof of Theorem 1 for Linear Costs. As mentioned in Section 3.1, there always exists an equilibrium. According to Proposition 8, if we start the algorithm with different initial values of $\bar{s}$, we can construct all the equilibria. Moreover, Proposition 7 implies that no matter what initial value $\bar{s}$ we use to start the algorithm, the algorithm constructs the same profile of strategies. Therefore, the equilibrium must be unique.

Assumption A2 of geometric and quadratic prize sequences plays a key role in the proof of Theorem 1. It guarantees Propositions 4 and 5, and the existence and uniqueness of pseudo strategies (see Lemma 6 in Appendix A). As we can see above, the proofs of Proposition 8 and Theorem 1 are directly hinged on these results. Possible challenges to relax the assumption are discussed in Section 6.

Having established the unique equilibrium, we can proceed to construct it. By the same argument from the proof for Theorem 1, Propositions 7 and 8 imply the following result.

Theorem 2 The algorithm in Section 3.2 constructs the unique equilibrium in every all-pay contest with a geometric or quadratic prize sequence and distinct linear costs. 
Recall that $\bar{s}_{i}^{*}$ and $\underline{s}_{i}^{*}$ are player $i$ 's maximum and minimum scores in the support of his equilibrium strategy. The proposition below discusses how the equilibrium reacts to shifts in the cost functions.

Proposition 9 If $c_{i}$ increases, $\bar{s}_{j}^{*}-\bar{s}_{k}^{*}$ remains the same for any $j, k<i$, and $\bar{s}_{j}^{*}-\bar{s}_{i}^{*}$ increases or remains the same for $j<i$.

There exists $\varepsilon>0$, such that if $c_{m+1}-c_{1}<\varepsilon$, we have $\bar{s}_{1}^{*}=\bar{s}_{2}^{*}=\ldots=\bar{s}_{m+1}^{*}$. There exists $\varepsilon^{\prime}>0$ such that if $\left(c_{i+1}-c_{i}\right)-\left(c_{i}-c_{i-1}\right)>\varepsilon^{\prime}$ for $i=2, \ldots, m$, we have $\bar{s}_{i+1}^{*}=\underline{s}_{i-1}^{*}$ for $i=2, \ldots, m$.

Let us explain the proposition. The first part of Proposition 9 implies that if a player becomes weaker, the difference between the maximum scores of any two players stronger than him remains the same, but the difference between a stronger player's maximum score and his increases or remains the same. The second part describes how the supports of equilibrium strategies overlap with each other. If all players have similar marginal costs, the supports of any two players' equilibrium strategies overlap, which means the intersection of the two supports has positive measure. On the other hand, if the cost sequence is convex enough, only two players compete "head-to-head" at any positive score. Moreover, the supports of $i-1$ and $i+1$ 's strategies do not overlap. For example, fix any geometric prize sequence with $n$ prizes and suppose the costs are $c_{i}=\gamma i^{2}$ for $i=1, \ldots, n$. Then, if the cost sequence is convex enough so that $\gamma>1 / 2$, the supports of $i-1$ and $i+1$ 's equilibrium strategies do not overlap.

\section{Nonlinear Costs}

This section generalizes both equilibrium uniqueness and construction for nonlinear costs. Nonlinear costs impose additional challenges. More precisely, the algorithm in Section 3.2 may not construct the equilibrium strategies if the costs are nonlinear. This is because, for linear costs, the starting value of the algorithm $\bar{s}$ only shifts the pseudo strategies. As a result, we can simply shift them in Step 2 to obtain the equilibrium payoffs. Since Proposition 7 no longer applies to nonlinear costs, a different starting value of $\bar{s}$ not only shifts the pseudo strategies but also changes the shape of them. Therefore, the algorithm in Section 3.2 may not construct the equilibrium.

However, a property of the algorithm allows us to modify it for nonlinear costs. Let us first explain the property, then present the modified algorithm. Given any equilibrium, suppose $\bar{s}_{1}^{*}$ is the highest score in the supports of all the strategies. Denote $u_{m+1}$ as player $m+1$ 's payoff constructed after Step 1. $(m+1)$. It is a property of the algorithm that $u_{m+1}<0$ if and only if the algorithm starts with a value $\bar{s}>\bar{s}_{1}^{*} \cdot{ }^{20}$ Therefore, given any guess $\bar{s}$, we know whether it is above or below the true value $\bar{s}_{1}^{*}$ by investigating the resulting $u_{m+1}$. Hence, we can construct a sequence of values converging to $\bar{s}_{1}^{*}$, and we can also construct a sequence of strategies that converge to the equilibrium.

Let us describe the modified algorithm. As in Section 3.2, we extend the definitions of the cost functions to negative scores such that $C_{i}^{\prime}(s)<C_{j}^{\prime}(s)$ for any $s \in \mathbb{R}$ and any $i, j$ with $i<j$. To

\footnotetext{
${ }^{20}$ This property is proved in Lemma 9 in Appendix C.
} 
start the algorithm, let $\bar{s}_{L}^{1}=0, \bar{s}_{U}^{1}=C_{1}^{-1}\left(v^{1}\right)$ and $\bar{s}^{1}=\left(\bar{s}_{L}^{1}+\bar{s}_{U}^{1}\right) / 2$. Taking $\bar{s}^{1}$ as the initial value, we proceed from Step 1.2 to Step 1.i, then proceed to Step 3.i and Step 4, skipping Step 2. Then, we move to Step 5, a new step. This step deals with three possibilities: First, if $u_{m+1}=0$, it means the initial guess $\bar{s}^{1}$ is correct and equals $\bar{s}_{1}^{*}$, so the algorithm ends. Second, if $u_{m+1}>0$, it means the initial guess $\bar{s}^{1}$ is too low, so we set $\bar{s}_{L}^{2}=\bar{s}^{1}$ and $\bar{s}_{U}^{2}=\bar{s}_{U}^{1}$ then go back to Step 1 with a higher initial value $\bar{s}^{2}=\left(\bar{s}_{L}^{2}+\bar{s}_{U}^{2}\right) / 2$, and proceed as above. Third, if $u_{m+1}<0$, the initial guess $\bar{s}^{1}$ is too high, so we update $\bar{s}_{L}^{2}=\bar{s}_{L}^{1}$ and $\bar{s}_{U}^{2}=\bar{s}^{1}$ and go back to Step 1 with a lower initial value $\bar{s}^{2}=\left(\bar{s}_{L}^{2}+\bar{s}_{U}^{2}\right) / 2$, and proceed as above. We call Step 1 to 5 an iteration.

With the modified algorithm, we can prove Theorem 1 for nonlinear costs in a similar way to linear costs. The proof is in Appendix D. In contrast to linear costs, the new algorithm does not stop in finite steps if it starts with $\bar{s}^{1} \neq \bar{s}_{1}^{*}$, therefore it only approximates the equilibrium. The proposition below characterizes the convergence rate of the approximation.

Theorem 3 Suppose $T$ is the number of iterations in the algorithm for nonlinear costs. Then, $\left|u_{i}^{*}-\hat{u}_{i, T}^{*}\right|=O\left(2^{-T}\right)$ for each player $i$, and $\left|G_{i}^{*}(s)-\hat{G}_{i, T}^{*}(s)\right|=O\left(2^{-T}\right)$ for each $s$ and $i$, where $\hat{u}_{i, T}^{*}$ is the payoff and $\hat{G}_{i, T}^{*}(s)$ is the strategy constructed by the Tth iteration of the algorithm. ${ }^{21}$

Multiple equilibria have been found in contests with a single prize (Baye et al. (1996)) or contests with symmetric players (Barut and Kovenock (1998)). In addition, the following example demonstrates that multiple equilibria also exist if players are not all symmetric and if there are multiple prizes. However, Theorem 1 enables us to select a unique equilibrium. The following example illustrates the selection, and Corollary 1 discusses the general case.

Example 2 Suppose that there are four players $(n=4)$ competing for two prizes $(m=2)$. The prize sequence is quadratic with $v^{1}=3$ and $v^{2}=1$. The players' marginal costs are $C_{1}^{\prime}(s)=1 / 10$, $C_{2}^{\prime}(s)=1, C_{3}^{\prime}(s)=C_{4}^{\prime}(s)=6 / 5$.

There are at least two types of Nash equilibria. First, there are equilibria with "type-asymmetric" participation. In an equilibrium, player 3 mixes over positive scores while player 4 , with the same costs as player 3 , always chooses zero. The equilibrium strategies are:

$$
\begin{aligned}
& G_{1}^{*}(s)=s / 2-3 / 8, \quad s \in[3 / 4,11 / 4] \\
& G_{2}^{*}(s)= \begin{cases}6 s / 5, & s \in[0,3 / 4) \\
s / 20+69 / 80, & s \in[3 / 4,11 / 4]\end{cases} \\
& G_{3}^{*}(s)=s+1 / 4, \quad s \in[0,3 / 4] \\
& G_{4}^{*}(s)=1
\end{aligned}
$$

If players 3 and 4 switch their strategies, we obtain another equilibrium of this type. Second, there

\footnotetext{
${ }^{21}$ Theorem 3 suggests a method to numerically calculate the equilibrium strategies. In practice, when we solve $\hat{G}_{i}^{*}$ from a differential equation system numerically, there could be small errors. Then we should ensure that the errors in the numerical solution are also controlled to be $O\left(2^{-T}\right)$, and the convergence rate would remain the same.
} 
is an equilibrium with "type-symmetric" participation. The equilibrium strategies are:

$$
\begin{aligned}
& G_{1}^{* *}(s)=s / 2-3 / 8, \quad s \in[3 / 4,11 / 4] \\
& G_{2}^{* *}(s)= \begin{cases}(s+1 / 4)^{-1 / 2} 6 s / 5, & s \in[0,3 / 4) \\
s / 20+69 / 80, & s \in[3 / 4,11 / 4]\end{cases} \\
& G_{3}^{* *}(s)=G_{4}^{* *}(s)=(s+1 / 4)^{1 / 2}, \quad s \in[0,3 / 4]
\end{aligned}
$$

The multiple equilibria impose several challenges. While the payoffs are the same across the equilibria, the prize allocations are not. For instance, player 4 does not win any prize in the first equilibrium, but he wins $v^{2}$ with positive probability in the second equilibrium. More importantly, the total expected scores (or performance levels) are also different across the equilibria. This makes it challenging to compare contest formats if the planner's objective is to maximize the total expected score (see Example 3).

However, Theorem 1 allows us to select a unique equilibrium. In particular, consider a sequence of contests in which player 4's cost is perturbed so that $C_{4, t}^{\prime}(s)>C_{3}^{\prime}(s)$ for all $s>0$ and $C_{4, t}^{\prime}(s)$ pointwise converges to $C_{4}^{\prime}(s)$. Then, Theorem 1 implies that there is a unique equilibrium for each $t$. Moreover, the sequence of equilibria converges to the equilibrium with type-asymmetric participation identified above. In other words, only the type-asymmetric participation is robust to perturbation in costs.

More generally, Corollary 1 allows us to select an equilibrium as a limit of the sequence of unique equilibria of nearby contests. ${ }^{22}$

Corollary 1 Consider a sequence of contests in which $C_{i}(s)-C_{j}(s)$ pointwise converges to zero. Then, i) if for player $k=i$ or $j$ we have $k \geq m+2$, then $u_{k}^{*}=0$ and $G_{k}^{*}(s)=1$ for $s \geq 0$ in each contest in the sequence; ii) if $i$ and $j<m+2, u_{i}^{*}-u_{j}^{*}$ converges to zero and $G_{i}^{*}(s)-G_{j}^{*}(s)$ pointwise converges to zero.

In Example 2, we illustrate that the robust participation to perturbation in costs may be typeasymmetric among weaker players $m+1, \ldots, n$. Property i) in Corollary 1 generalizes this selection. Moreover, property ii) in the corollary discusses the robust equilibrium strategies for stronger players $1, \ldots, m+1$. The selected equilibrium has $m+1$ players who mix over positive scores, and among these players, those with the same cost use the same strategy. Thus, the equilibrium selection is unique if we do not distinguish players $i, j<m+2$ with the same costs. For example, consider a contest with one prize and three players with marginal costs $0<c_{1} \leq c_{2} \leq c_{3}$. Figure 5 schematically illustrates the equilibrium selection. In particular, fix $c_{1}$ and $c_{3}$, we have $c_{2} \in\left[c_{1}, c_{3}\right]$. If $c_{2}$ is at the upper boundary $c_{3}$, there are multiple equilibria represented by the bold vertical interval in the figure. However, if $c_{2}$ is slightly lower than $c_{3}$, there is a unique equilibrium.

\footnotetext{
${ }^{22}$ This paper assumes ordered marginal costs, so the perturbed marginal costs should also be ordered. This is trivially satisfied if we only consider linear costs. However, if the perturbed marginal costs are not ordered, the examples by Siegel (2009) illustrate that there could be multiple equilibria.
} 
Therefore, as $c_{2}$ increases to $c_{3}$, the limit of the unique equilibrium converges, along the curve in Figure 5 , to one of the multiple equilibria. ${ }^{23}$

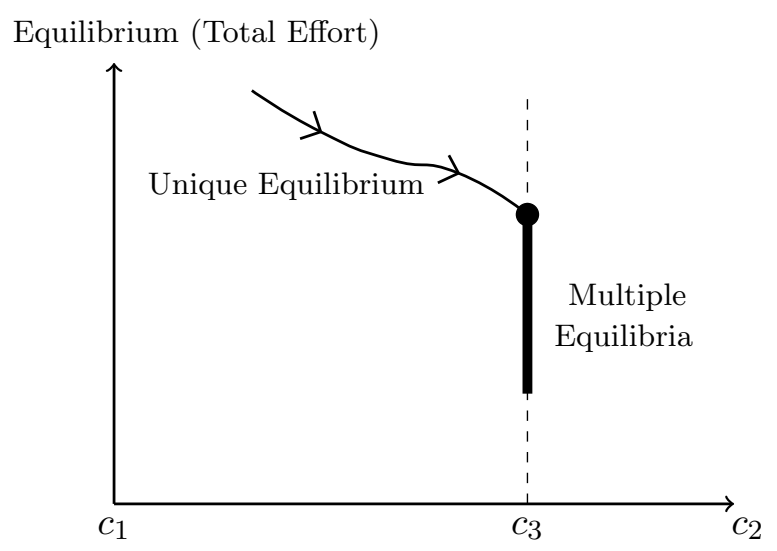

Figure 5: Equilibrium Selection

\section{Applications}

\subsection{Tracking in Schools}

Consider a situation in which a school wants to assign a group of students to different classrooms. Should the school group students with similar abilities together - a practice known as "tracking" - so that high ability students are grouped together or should the school have mixed classrooms in which students of all abilities are grouped together? Tracking is a controversial topic in the economics of education and there has been a long debate on the issue from many different perspectives: students' achievement, equity, and even morality. ${ }^{24}$ Evidence on the effect of tracking on student achievement is mixed (see Betts and Shkolnik 2000). Moreover, different countries adopt different policies. Tracking is quite common in German and US schools. In contrast, tracking was explicitly discontinued in China in 2006. ${ }^{25}$ In this section, we examine the pure competitive effects of tracking and ask whether or not it enhances students' overall effort levels when students' grades/rewards depend on their relative performance.

Suppose there are two classrooms with $2 n$ seats in each classroom. Each classroom has a prize sequence. In order to illustrate the distributional effect of prizes, we assume the sequences are exogenous and the same across classrooms. ${ }^{26}$ The sequences are geometric or quadratic, and each has $2 n$ positive prizes. There are $2 n H$-type students and $2 n L$-type students. The $H$-type students have a marginal cost of $c_{H}$, the $L$-type students have a marginal cost of $c_{L}$, and $0<c_{H}<c_{L}$. If

\footnotetext{
${ }^{23}$ Baye et al. (1996) demonstrate that, if there is only one prize, the equilibrium selected here has the highest total expected effort.

${ }^{24}$ For instance, Lazear (2001) considers the effect of tracking on class disruption caused by misbehaving students. Duflo et al. (2011) study the effect on instructions, and demonstrate in an experimental study that, if students are tracked, the classes are more homogeneous and therefore could be easier to teach.

${ }^{25}$ Policies that forbade tracking started in the 1990s, and a national law was passed in 2006.

${ }^{26}$ In another paper, Xiao (2013) considers endogenous prize sequences, and finds that tracking is better than mixing.
} 
students are tracked, $H$-type students are assigned to one classroom and $L$-type students are assigned to another. If students are mixed, every classroom contains $n$ students of each type. The students in each classroom compete in an all-pay contest.

Notice that some players have the same type, so the setup in this section is different from Section 2. However, Theorem 1 and Corollary 1, which are obtained with distinct players, are important for this section. Let us explain why. First, an important characteristic of student competition is heterogeneous returns to performance ranking, and usually increasing returns to ranking. Without our results on GPS or QPS, we cannot capture this characteristic, and therefore cannot study the effects of convexity of prize sequences. Second, multiple equilibria impose an additional challenge. We first present our results on the effects of convexity of prize sequences, then show how our results tackle the challenge of multiple equilibria.

To illustrate the contribution of our equilibrium characterization, let us first discuss what results we can obtain with the existing equilibrium characterizations. On the one hand, if we have an arithmetic prize sequence (e.g. Bulow and Levin 2006) - the least convex prize sequence, we can verify that tracking is better than mixing. On the other hand, if each classroom has a single prize (e.g. Baye et al. 1996) - a very convex prize sequence, mixing is better than tracking. Our results on GPS and QPS allow us to fill the gap between these two extreme cases.

Proposition 10 Assume that the prize sequence under tracking is the one under mixing for each classroom, and assume that the prize sequence is either geometric or quadratic. Then, mixing results in higher total expected effort than tracking if and only if the prize sequence is convex enough, that is, $\alpha>\alpha^{\prime}$ in GPS or $\beta>\beta^{\prime}$ in $Q P S$ for some $\alpha^{\prime} \geq 1$ and $\beta^{\prime} \geq 0$.

The proof of the proposition is in Appendix E.1. Why does the convexity of prize sequence matter? Tracking has an advantage of facilitating greater competition in each classroom because it assigns students of similar abilities together. However, tracking also has a disadvantage. It does not use the highest prizes to motivate the best students. If the prize sequence is linear, the value of the lower prize is not too small. Therefore, the advantage dominates, and tracking is better than mixing. On the other hand, if the prize sequence is convex enough, the lower prizes are very small. If the students are tracked, only half of the prize money is used to motivate $H$-type students. However, if the students are mixed, most of the prize money is used to motivate $H$-type students. Hence, the disadvantage of tracking dominates its advantage, and tracking is worse than mixing. ${ }^{27}$

Moreover, multiple equilibria may cause a second challenge. Example 2 shows that there could be multiple equilibria, but would the multiple equilibria affect comparison of mixing and tracking? We first demonstrate the challenge in an example, then show how our results tackle it.

Example 3 Suppose there are eight students: two with $H$-type, two with $M$-type and four with $L$-type. The marginal costs for type $H, M, L$ are $c_{H}=0.8, c_{M}=1, c_{L}=2$ respectively, and the prizes are $v^{1}=3.94, v^{2}=4-v^{1}$.

\footnotetext{
${ }^{27}$ The number of prizes is also important. If there are fewer prizes, mixing could be better than tracking. The winner-take-all prize structure describes many competitions, but it is not a good approximation for the competition among students. This is because ranking may matter to all students in their competition, even for those close to the bottom.
} 
If the students are tracked, the $H$-type and $M$-type students are assigned to one classroom and the $L$-type students are grouped into another classroom. The students in each classroom compete in an all-pay contest for the prizes $v^{1}=3.94, v^{2}=4-v^{1}$. The algorithm implies that each student's equilibrium payoff is zero, and the $M$-type students choose 0 score in the equilibrium. Therefore the total expected effort is $\Pi_{\text {track }}=\left(v^{1}+v^{2}\right) / c_{H}+\left(v^{1}+v^{2}\right) / c_{L}=7$ in every equilibrium.

If the students are mixed, each classroom has one $H$-type, one $M$-type and two $L$-type students. They also compete in an all-pay contest for the prizes $v^{1}=3.94, v^{2}=4-v^{1}$. There are at least two equilibria. In the first equilibrium, only one $L$-type student is active and the other chooses zero effort. Again the algorithm implies that the supports of $H$-type students' and $M$-type students' strategies do not overlap. Using the algorithm, we can calculate the equilibrium strategies, the total expected effort $\Pi_{\text {mix }}$ and the difference in total effort

$$
\Pi_{\text {track }}-\Pi_{m i x}=-1.7 \times 10^{-5}
$$

In another equilibrium, the supports are the same as in the first equilibrium, but two $L$-type

students are active instead of one. Similarly we can calculate the total expected effort $\hat{\Pi}_{m i x}$, and the difference in total effort becomes

$$
\Pi_{\text {track }}-\hat{\Pi}_{m i x}=1.46 \times 10^{-5}
$$

Hence, the comparison between tracking and mixing depends on equilibrium selection. However, Corollary 1 implies that only (9) is robust to perturbation in the costs.

\subsection{Winner-Take-All?}

Consider a situation in which the designer of a contest has a fixed amount of prize money, and she wants to choose the optimal prize structure to maximize the total expected score (performance). Is it optimal for the designer to adopt a winner-take-all prize structure, in which the whole amount is won by the highest-ranked player, or should the total amount be split into two or more prizes?

Moldovanu and Sela (2001) consider a contest with incomplete information. One of their results is that the winner-take-all prize structure is optimal if the players are ex ante symmetric and the costs are linear. Intuitively, a single prize maximizes the difference between winning and losing, therefore it induces the most intense competition. However, this insight does not apply to contests with asymmetric players and complete information. To see why, note that if the players are ex ante symmetric, the number of prizes does not affect participation because every player always participates in the symmetric equilibrium. In contrast, if the players are asymmetric and information is complete, more prizes could encourage more players to participate and therefore induce more competition. This point can be illustrated in our setup.

Similar intuition is studied in different setups. For instance, Szymanski and Valletti (2005) consider a Tullock contest with one strong player and two weaker players. They show that $v^{1}=3 v^{2}$ maximizes the total effort if the cost of the stronger player converges to zero. Cohen and Sela (2008) 
study an all-pay auction with three players, and they show that if one player values the second prize slightly higher than the other two, two prizes result in higher revenue than a single prize. Olszewski and Siegel (2014) find that a prize sequence with homogeneous prizes maximizes the total expected effort in an all-pay contest if the number of players goes to infinity.

However, there are many questions that have not been answered. For example, are there other prize sequences better than winner-take-all if there are more than three players? If there are, how do they compare with each other? The proposition below answers these questions. Our algorithm to construct the equilibrium is important for the proposition.

Proposition 11 Suppose $c_{2}=\ldots=c_{n}>0$, and let $\Pi_{H P S}^{*}$ be the supremum of total expected effort with homogeneous prize sequence of multiple positive prizes, $\Pi_{G P S}^{*}$ be the counterpart with GPS, $\Pi_{Q P S}^{*}$ with QPS, and $\Pi_{W T A}$ with the winner-take-all prize structure. ${ }^{28}$ Then, we have

$$
\lim _{c_{1} \rightarrow 0} \Pi_{H P S}^{*}>\lim _{c_{1} \rightarrow 0} \Pi_{Q P S}^{*}>\lim _{c_{1} \rightarrow 0} \Pi_{G P S}^{*}>\lim _{c_{1} \rightarrow 0} \Pi_{W T A}
$$

The proof is in Appendix E.2. According to the proposition, a prize structure with multiple prizes dominates a single prize. Let us explain why. If $c_{1}$ is small, player 1 is very strong relative to others, so he does not exert much effort despite the amount of the first prize. As a result, if we move some money from the first prize to the lower ones, the effort of player 1 does not change much. In contrast, if the lower prizes are not zero, the weaker players $2, \ldots, n$ mainly compete for the second and lower prizes, where they face intense competition from $n-2$ identical opponents. As a result, multiple prizes can enhance the weaker players' effort and therefore induce higher total effort.

\section{Conclusion}

In this paper, we studied a complete information model of all-pay contests with asymmetries among players and (two classes) of convex prize sequences. While it would be desirable to study a similar environment under incomplete information, the problems associated with multiple prizes and asymmetric players under incomplete information are well known from auction theory. For instance, even with symmetric players very little is known about discriminatory (pay-as-you-bid) auctions for the sale of multiple units. Similar difficulties arise when considering all-pay auctions with multiple prizes. ${ }^{29}$ The complete information setting allows us to study environments that, as yet, cannot be studied under an incomplete information setting.

Our results require the prize sequence to be either quadratic or geometric. Although, many common prize structures can be well-approximated by one of these specifications, one would still

\footnotetext{
${ }^{28}$ The assumption $c_{2}=\ldots=c_{n}$ can be relaxed to allow small differences among these costs. That is, given $c_{2}>0$, the difference $\left|c_{n}-c_{2}\right|$ goes to zero. Then, we need to replace $\lim _{c_{1} \rightarrow 0}$ with $\lim _{c_{1} \rightarrow 0,\left|c_{n}-c_{2}\right| \rightarrow 0}$ in (10). Corollary 1 implies that the order of limits does not matter.

${ }^{29}$ Studies of similar cases have shown that there is a unique equilibrium in asymmetric all-pay auctions with two players (Amann and Leininger 1996, Lizzeri and Persico 2000). The complexity in the case of more than two players is demonstrated by Parreiras and Rubinchik (2010).
} 
like to extend the results of this paper to general convex prize sequences. The quadratic/geometric specification plays a key role in the proofs - it guarantees the existence and uniqueness of pseudo strategies (see Lemma 6 in Appendix A), and Propositions 4 and 5. The main difficulty in extending our analysis to general convex sequences is Lemma 5, a property of a nonlinear equation system, which is repeatedly used in Appendix A. To establish this property, we need to sign a determinant of high dimension, which is a very challenging problem even with geometric or quadratic prize sequences (see the supplementary note). Without the assumption, how to sign the determinants remains an open question.

If we restrict our attention to three players, the results in this paper include all convex prize sequences, and it would be interesting to explore the potential of our approach to study concave prize sequences. Since the focus of this paper is convex prize sequences, we leave it to future research.

We hope to explore some extensions of the model. It would be interesting to investigate, more generally, what an optimal prize sequence looks like. Must it be convex? Does the uniqueness result hold for general convex (possibly non-geometric and non-quadratic) prize sequences? These and other questions will be explored in subsequent work.

\section{References}

Amann, E. and Leininger, W. (1996), "Asymmetric All-Pay Auctions with Incomplete Information: the Two-Player Case", Games and Economic Behavior, 14, pp. 118. [26]

Barut, Y. and Kovenock, D. (1998), "The Symmetric Multiple Prize All-Pay Auction with Complete Information", European Journal of Political Economy, 14, pp. 627-644. [3, 4, and 21]

Baye, M. R.; Kovenock, D. and de Vries, C. G. (1996), "The All-Pay Auction with Complete Information", Economic Theory, 8, pp. 291-305. [3, 4, 21, 23, and 24]

Betts, J. R. and Shkolnik, J. L. (2000), "Key Difficulties in Identifying the Effects of Ability Grouping on Student Achievement", Economics of Education Review, 19, pp. 21-26. [23]

Bulow, J. and Levin, J. (2006), "Matching and Price Competition", American Economic Review, 96 , pp. 652-668. [3, 4, 6, and 24]

Clark, D. J. and Riis, C. (1998), "Competition Over More than One Prize", American Economic Review, 88, pp. 276-289. [3 and 4]

Cohen, C. and Sela, A. (2008), "Allocation of Prizes in Asymmetric All-pay Auctions", European Journal of Political Economy, 24, pp. 123-132. [25]

Duflo, E., Dupas, P. and Kremer, M. (2011), "Peer Effects and the Impacts of Tracking: Evidence from a Randomized Evaluation in Kenya", American Economic Review, 101, pp. 1739-1774. [23] 
González-Díaz, J. and Siegel, R. (2013), "Matching and Price Competition: Beyond Symmetric Linear Costs", International Journal of Game Theory, 42, pp. 835-844. [4]

Konrad, K. A. (2009), Strategy and Dynamics in Contests, Oxford University Press. [3]

Lazear, E. P. (2001), "Educational Production", Quarterly Journal of Economics, 116, pp. 777-803. $[23]$

Lizzeri, A. and Persico, N. (2000), "Uniqueness and Existence of Equilibrium in Auctions with a Reserve Price", Games and Economic Behavior, 30, pp. 83-114. [26]

Moldovanu, B. and Sela, A. (2001), "The Optimal Allocation of Prizes in Contests", American Economic Review, 91, pp. 542-558. [3 and 25]

Olszewski, W. and Siegel, R. (2015), "Large Contests", Econometrica, forthcoming. [4]

Olszewski, W. and Siegel, R. (2014), "Effort-Maximizing Contests", Mimeo, Northwestern University. [26]

Olver, P. (2007), Applied Mathematics Lecture Notes, Schools of Mathematics, University of Minnesota. [36 and 39]

Parreiras, S. O. and Rubinchik, A. (2010), "Contests with Three or More Heterogeneous Agents", Games and Economic Behavior, 68, pp. 703-715. [26]

Pugh, C. C. (2003), Real Mathematical Analysis, New York: Springer-Verlag. [39]

Siegel, R. (2009), "All-Pay Contests", Econometrica, 77, pp. 71-92. [4, 6, 22, and 29]

Siegel, R. (2010), "Asymmetric Contests with Conditional Investments", American Economic Review, 100, pp. 2230-2260. [3, 4, and 5]

Siegel, R. (2014), "Asymmetric Contests with Interdependent Valuations", Journal of Economic Theory, 153, pp. 684-702. [4]

Szymanski, S. and Valletti, T. (2005), "Incentive Effects of Second Prizes", European Journal of Political Economy, 21, pp. 467-481. [25]

Xiao, J. (2013), "Ability Grouping in All-Pay Contests", Mimeo, University of Melbourne. [23]

\section{Appendix}

\section{A Equilibrium Properties}

This appendix contains proofs for the results in Section 3.1. In what follows, we first define a function $W_{\mathcal{P}}$, then Lemma 1 discusses some properties of the function. After that, Lemma 2 uses the properties to prove the first "well known" property in Section 3.1. 
For any subset $\mathcal{P} \subseteq \mathcal{N}$, define a function $W_{\mathcal{P}}:[0,1]^{|\mathcal{P}|-1} \times[0, \infty)^{|\mathcal{P}|} \rightarrow[0, \infty)$ that takes the form

$$
W_{\mathcal{P}}\left(\mathbf{x}_{-j}, \mathbf{v}\right)=\sum_{k=1}^{|\mathcal{P}|}\left(v^{(k)} \sum_{P_{k-1} \in \mathcal{D}_{k-1}}\left(\prod_{i \in P_{k-1}}\left(1-x_{i}\right) \prod_{i^{\prime} \in(\mathcal{P} \backslash\{j\}) \backslash P_{k-1}} x_{i^{\prime}}\right)\right)
$$

where $j \in \mathcal{P}, \mathbf{x}_{-j}=\left(x_{i}\right)_{i \in \mathcal{P} \backslash\{j\}} \in[0,1]^{|\mathcal{P}|-1}, \mathbf{v} \in[0, \infty)^{|\mathcal{P}|}, v^{(k)}$ is the $k$ th highest element in $\mathbf{v}$, and $\mathcal{D}_{k-1}=\left\{P_{k-1} \mid P_{k-1} \subseteq \mathcal{P} \backslash\{j\}\right.$ and $\left.\left|P_{k-1}\right|=k-1\right\}$.

Lemma 1 For any $\mathcal{P} \subseteq \mathcal{N}$, function $W_{\mathcal{P}}\left(\mathbf{x}_{-i}, \mathbf{v}\right)$ is symmetric in the variables of $\mathbf{x}_{-i}$; it is linear in $\mathbf{v}$; and it is strictly increasing in a variable $x_{j}$ in $\mathbf{x}_{-i}$ if $\mathbf{x}_{-i, j}=\left(x_{k}\right)_{k \in \mathcal{P} \backslash\{i, j\}}$ is not a zero vector.

Proof. It is straightforward to show that $W_{\mathcal{P}}\left(\mathbf{x}_{-i}, \mathbf{v}\right)$ is symmetric in the variables in $\mathbf{x}_{-i}$ and linear in $\mathbf{v}$. To see why it is increasing in $x_{j}$, notice

$$
W_{\mathcal{P}}\left(\mathbf{x}_{-i}, \mathbf{v}\right)=G_{j}(s) W_{\mathcal{P}}\left(\mathbf{x}_{-i, j}, \overline{\mathbf{v}}\right)+\left(1-x_{j}\right) W_{\mathcal{P}}\left(\mathbf{x}_{-i, j}, \underline{\mathbf{v}}\right)
$$

where $\overline{\mathbf{v}}$ is $\mathbf{v}$ with one of the lowest prizes excluded, and $\underline{\mathbf{v}}$ is $\mathbf{v}$ with one of the highest prize excluded. Differentiating both sides of (11) with respect to $x_{j}$, we obtain

$$
\partial W_{\mathcal{P}}\left(\mathbf{x}_{-i}, \mathbf{v}\right) / \partial x_{j}=W_{\mathcal{P}}\left(\mathbf{x}_{-i, j}, \overline{\mathbf{v}}\right)-W_{\mathcal{P}}\left(\mathbf{x}_{-i, j}, \underline{\mathbf{v}}\right)=W_{\mathcal{P}}\left(\mathbf{x}_{-i, j}, \overline{\mathbf{v}}-\underline{\mathbf{v}}\right)
$$

where the second equality comes from the linearity in v. Assumption A2 implies that the prizes in $\mathbf{v}$ are distinct, so $\overline{\mathbf{v}}-\underline{\mathbf{v}}$ represents a sequence of positive prizes. Given these prizes, the expected winnings $W_{\mathcal{P}}\left(\mathbf{x}_{-i, j}, \overline{\mathbf{v}}-\underline{\mathbf{v}}\right)$ must be positive if $\mathbf{x}_{-i, j}$ is not a zero vector. Hence, (12) implies $\partial W_{\mathcal{P}}\left(\mathbf{x}_{-i}, \mathbf{v}\right) / \partial x_{j}>0$.

Lemma 2 (Participation) Player $i>m+1$ assigns probability one to score 0.

Proof. The Zero Lemma of Siegel (2009) also applies to heterogeneous prizes. If we replace the probability of winning (one of the identical prizes) with the probability of winning at least one prize (of the heterogeneous prizes), his proof of the lemma extends to our context. The Zero Lemma implies that at least $n-m$ players have zero expected payoff.

We claim that $u_{i}^{*} \geq u_{j}^{*}$ for $j>i$. To see why, let $\bar{s}_{j}^{*}$ be the highest score in the support of $j$ 's equilibrium strategy. Player $i$ can guarantee himself a payoff no less than $u_{j}^{*}$ by choosing a score slightly higher than $\bar{s}_{j}^{*}$, so $u_{i}^{*} \geq u_{j}^{*}$. Therefore, the Zero Lemma implies $u_{i}^{*}=0$ for $i \geq m+1$.

Suppose player $i>m+1$ assigns positive probability to a set of positive scores, so $\bar{s}_{i}^{*}>0$. Then, we have

$$
\mathbf{G}_{-(m+1)}^{*}\left(\bar{s}_{i}^{*}\right)=\left(\mathbf{G}_{-(m+1), i}^{*}\left(\bar{s}_{i}^{*}\right), G_{i}^{*}\left(\bar{s}_{i}^{*}\right)\right) \geq\left(\mathbf{G}_{-(m+1), i}^{*}\left(\bar{s}_{i}^{*}\right), G_{m+1}^{*}\left(\bar{s}_{i}^{*}\right)\right)=\mathbf{G}_{-i}^{*}\left(\bar{s}_{i}^{*}\right)
$$

where the inequality comes from $G_{i}^{*}\left(\bar{s}_{i}^{*}\right)=1$. Given others' equilibrium strategies, if player $m+1$ 
chooses $\bar{s}_{i}^{*}$, his expected payoff is

$$
\begin{aligned}
W_{\mathcal{N}}\left(\mathbf{G}_{-(m+1)}^{*}\left(\bar{s}_{i}^{*}\right), \mathbf{v}\right)-C_{m+1}\left(\bar{s}_{i}^{*}\right) & \geq W_{\mathcal{N}}\left(\mathbf{G}_{-i}^{*}\left(\bar{s}_{i}^{*}\right), \mathbf{v}\right)-C_{m+1}\left(\bar{s}_{i}^{*}\right) \\
& >W_{\mathcal{N}}\left(\mathbf{G}_{-i}^{*}\left(\bar{s}_{i}^{*}\right), \mathbf{v}\right)-C_{i}\left(\bar{s}_{i}^{*}\right) \\
& =u_{i}^{*} \geq 0
\end{aligned}
$$

where the first inequality is because of (13) and the monotonicity in Lemma 1, and the second inequality comes from Assumption A1. Note that we also use Assumption A2 to prove Lemma 1. Hence, player $m+1$ can guarantee himself a positive payoff by choosing $\bar{s}_{i}^{*}$, so $u_{m+1}^{*}>0$. Recall that the Zero Lemma implies $u_{m+1}^{*}=0$, so we have a contradiction.

In what follows, we first introduce two properties in Lemmas 3 and 4 , then use them to prove Propositions 1 to 3 .

Lemma 3 In any equilibrium, $\bar{s}_{j}^{*} \leq \bar{s}_{i}^{*}$ if $i<j$. That is, the highest score chosen by a stronger player is no lower than that chosen by a weaker player.

Proof. Suppose $\bar{s}_{i}^{*}<\bar{s}_{j}^{*}$. First, the definition of equilibrium implies that player $i$ 's payoff at $\bar{s}_{i}^{*}$ should not be lower than that at $\bar{s}_{j}^{*}$. That is,

$$
W_{\mathcal{N}}\left(\mathbf{G}_{-i}^{*}\left(\bar{s}_{i}^{*}\right), \mathbf{v}\right)-C_{i}\left(\bar{s}_{i}^{*}\right) \geq W_{\mathcal{N}}\left(\mathbf{G}_{-i}^{*}\left(\bar{s}_{j}^{*}\right), \mathbf{v}\right)-C_{i}\left(\bar{s}_{j}^{*}\right)
$$

Hereafter, we omit the subscript of $W_{\mathcal{N}}$ and write $W$ for short. Since $\bar{s}_{i}^{*}<\bar{s}_{j}^{*}$, we have $G_{j}^{*}\left(\bar{s}_{j}^{*}\right)=$ $G_{i}^{*}\left(\bar{s}_{j}^{*}\right)=1$. Similar to (13), we have $\mathbf{G}_{-i}^{*}\left(\bar{s}_{j}^{*}\right)=\mathbf{G}_{-j}^{*}\left(\bar{s}_{j}^{*}\right)$. Substituting the equation into (15), we obtain

$$
W\left(\mathbf{G}_{-i}^{*}\left(\bar{s}_{i}^{*}\right), \mathbf{v}\right)-C_{i}\left(\bar{s}_{i}^{*}\right) \geq W\left(\mathbf{G}_{-j}^{*}\left(\bar{s}_{j}^{*}\right), \mathbf{v}\right)-C_{i}\left(\bar{s}_{j}^{*}\right)
$$

or

$$
W\left(\mathbf{G}_{-j}^{*}\left(\bar{s}_{j}^{*}\right), \mathbf{v}\right)-W\left(\mathbf{G}_{-i}^{*}\left(\bar{s}_{i}^{*}\right), \mathbf{v}\right) \leq C_{i}\left(\bar{s}_{j}^{*}\right)-C_{i}\left(\bar{s}_{i}^{*}\right)
$$

Second, the definition of equilibrium implies that player $j$ 's payoff at $\bar{s}_{j}^{*}$ should not be lower than that at $\bar{s}_{i}^{*}$. That is,

$$
W\left(\mathbf{G}_{-j}^{*}\left(\bar{s}_{j}^{*}\right), \mathbf{v}\right)-C_{j}\left(\bar{s}_{j}^{*}\right) \geq W\left(\mathbf{G}_{-j}^{*}\left(\bar{s}_{i}^{*}\right), \mathbf{v}\right)-C_{j}\left(\bar{s}_{i}^{*}\right)
$$

Since $\bar{s}_{i}^{*}<\bar{s}_{j}^{*}$, we have $G_{j}^{*}\left(\bar{s}_{i}^{*}\right)<G_{i}^{*}\left(\bar{s}_{i}^{*}\right)$. Therefore, following the same argument to obtain (13) and (14), we have

$$
W\left(\mathbf{G}_{-j}^{*}\left(\bar{s}_{i}^{*}\right), \mathbf{v}\right)>W\left(\mathbf{G}_{-i}^{*}\left(\bar{s}_{i}^{*}\right), \mathbf{v}\right)
$$

Hence, (17) and (18) imply

$$
W\left(\mathbf{G}_{-j}^{*}\left(\bar{s}_{j}^{*}\right), \mathbf{v}\right)-C_{j}\left(\bar{s}_{j}^{*}\right)>W\left(\mathbf{G}_{-i}^{*}\left(\bar{s}_{i}^{*}\right), \mathbf{v}\right)-C_{j}\left(\bar{s}_{i}^{*}\right)
$$


or

$$
W\left(\mathbf{G}_{-j}^{*}\left(\bar{s}_{j}^{*}\right), \mathbf{v}\right)-W\left(\mathbf{G}_{-i}^{*}\left(\bar{s}_{i}^{*}\right), \mathbf{v}\right)>C_{j}\left(\bar{s}_{j}^{*}\right)-C_{j}\left(\bar{s}_{i}^{*}\right)
$$

Notice that Assumption A1 implies $C_{i}\left(\bar{s}_{j}^{*}\right)-C_{i}\left(\bar{s}_{i}^{*}\right)<C_{j}\left(\bar{s}_{j}^{*}\right)-C_{j}\left(\bar{s}_{i}^{*}\right)$, so (16) and (19) contradict with each other. As a result, $\bar{s}_{j}^{*} \leq \bar{s}_{i}^{*}$.

Lemma 4 In any equilibrium, if $i<j$, we have $u_{i}^{*}+C_{i}(s) \geq u_{j}^{*}+C_{j}(s)$ for $s \leq \bar{s}_{j}^{*}$. That is, the difference between the equilibrium payoffs is no larger than the difference in the costs.

Proof. Lemma 3 implies $\bar{s}_{j}^{*} \leq \bar{s}_{i}^{*}$. An analogue to (18) gives us

$$
W\left(\mathbf{G}_{-i}^{*}\left(\bar{s}_{j}^{*}\right), \mathbf{v}\right) \geq W\left(\mathbf{G}_{-j}^{*}\left(\bar{s}_{j}^{*}\right), \mathbf{v}\right)
$$

The definition of equilibrium implies player $i$ should not receive a higher payoff at $\bar{s}_{j}^{*}$ than $u_{i}^{*}$, or

$$
W\left(\mathbf{G}_{-i}^{*}\left(\bar{s}_{j}^{*}\right), \mathbf{v}\right)-C_{i}\left(\bar{s}_{j}^{*}\right) \leq u_{i}^{*}
$$

Therefore, we have

$$
u_{i}^{*}+C_{i}\left(\bar{s}_{j}^{*}\right) \geq W\left(\mathbf{G}_{-i}^{*}\left(\bar{s}_{j}^{*}\right), \mathbf{v}\right) \geq W\left(\mathbf{G}_{-j}^{*}\left(\bar{s}_{j}^{*}\right), \mathbf{v}\right)=u_{j}^{*}+C_{j}\left(\bar{s}_{j}^{*}\right)
$$

where the first inequality comes from (21), the second comes from (20) and the equality comes from the definition of equilibrium.

For any $s \leq \bar{s}_{j}^{*}$, we have

$$
u_{i}^{*}+C_{i}(s)-\left(u_{j}^{*}+C_{j}(s)\right)=u_{i}^{*}-u_{j}^{*}-\left(C_{j}(s)-C_{i}(s)\right) \geq u_{i}^{*}-u_{j}^{*}-\left(C_{j}\left(\bar{s}_{j}^{*}\right)-C_{i}\left(\bar{s}_{j}^{*}\right)\right) \geq 0
$$

where the first inequality comes from Assumption A1 and the last comes from (22).

Proof of Proposition 1 (Nested Gaps). We prove by contradiction. Suppose there is a score such that the proposition is violated. Let $t$ be the supremum of such scores, so $t \equiv \sup \{s \mid$ $\left.i, j \in \mathcal{P}^{*}(s), i \notin \mathcal{A}^{*}(s), j \in \mathcal{A}^{*}(s)\right\}$. We construct a contradiction in three steps.

Step I. This step shows $G_{i}^{*}(t) \leq G_{j}^{*}(t)$. Define $t_{1} \equiv \inf \left\{s>t \mid i, j \in \mathcal{A}^{*}(s)\right\}$. By the definition of $\mathcal{A}^{*}\left(t_{1}\right)$, players $i$ and $j$ receive their equilibrium payoff by choosing $t_{1}$. That is,

$$
\begin{aligned}
W\left(\mathbf{G}_{-i}^{*}\left(t_{1}\right), \mathbf{v}\right)-C_{i}\left(t_{1}\right) & =u_{i}^{*} \\
W\left(\mathbf{G}_{-j}^{*}\left(t_{1}\right), \mathbf{v}\right)-C_{j}\left(t_{1}\right) & =u_{j}^{*}
\end{aligned}
$$

Therefore, we have

$$
W\left(\mathbf{G}_{-i}^{*}\left(t_{1}\right), \mathbf{v}\right) \geq u_{i}^{*}+C_{i}\left(t_{1}\right) \geq u_{j}^{*}+C_{j}\left(t_{1}\right) \geq W\left(\mathbf{G}_{-j}^{*}\left(t_{1}\right), \mathbf{v}\right)
$$

where the first and third inequalities come from (23) and (24), and the second comes from Lemma 4. Notice that $\mathbf{G}_{-i}^{*}\left(t_{1}\right)=\left(\mathbf{G}_{-i, j}^{*}\left(t_{1}\right), G_{j}^{*}\left(t_{1}\right)\right)$ and $\mathbf{G}_{-j}^{*}\left(t_{1}\right)=\left(\mathbf{G}_{-i, j}^{*}\left(t_{1}\right), G_{i}^{*}\left(t_{1}\right)\right)$, so (25) and the 
monotonicity in Lemma 1 imply

$$
G_{i}^{*}\left(t_{1}\right) \leq G_{j}^{*}\left(t_{1}\right)
$$

Note that Lemma 1 relies on Assumption A2, and Lemma 4 relies on Assumption A1. The definitions of $t$ and $t_{1}$ imply that for any $s \in\left(t, t_{1}\right)$, we have either $i, j \in \mathcal{A}^{*}(s)$ or $i \in \mathcal{A}^{*}(s), j \notin \mathcal{A}^{*}(s)$. In either case, $G_{j}^{*}(s)-G_{i}^{*}(s)$ is non-increasing, so

$$
G_{j}^{*}(t)-G_{i}^{*}(t) \geq G_{j}^{*}\left(t_{1}\right)-G_{i}^{*}\left(t_{1}\right) \geq 0
$$

where the last inequality comes from (26). Therefore, we verify that $G_{i}^{*}(t) \leq G_{j}^{*}(t)$.

Step II. This step shows

$$
W\left(\mathbf{G}_{-i}^{*}(t), \mathbf{v}\right)-W\left(\mathbf{G}_{-i}^{*}\left(t_{0}\right), \mathbf{v}\right) \geq W\left(\mathbf{G}_{-j}^{*}(t), \mathbf{v}\right)-W\left(\mathbf{G}_{-j}^{*}\left(t_{0}\right), \mathbf{v}\right)
$$

where $t_{0} \equiv \inf \left\{s<t \mid G_{i}^{*}\left(s^{\prime}\right)=G_{i}^{*}(t)\right.$ for $\left.s^{\prime} \in(s, t)\right\}$.

We first claim that

$$
G_{i}^{*}\left(t_{0}\right) \leq G_{j}^{*}\left(t_{0}\right)
$$

To see why, suppose $G_{i}^{*}\left(t_{0}\right)>G_{j}^{*}\left(t_{0}\right)$. Then, the definition of $t_{0}$ implies $i \in \mathcal{A}^{*}\left(t_{0}\right)$, so player $i$ receives his equilibrium payoff by choosing $t_{0}$. As a result,

$$
u_{i}^{*}+C_{i}\left(t_{0}\right)=W\left(\mathbf{G}_{-i}^{*}\left(t_{0}\right), \mathbf{v}\right)<W\left(\mathbf{G}_{-j}^{*}\left(t_{0}\right), \mathbf{v}\right) \leq u_{j}^{*}+C_{j}\left(t_{0}\right)
$$

where the first inequality comes from $G_{i}^{*}\left(t_{0}\right)>G_{j}^{*}\left(t_{0}\right)$ and the second is because $t_{0}$ should not be a profitable deviation for $j$. The above inequality implies $u_{i}^{*}+C_{i}\left(t_{0}\right)<u_{j}^{*}+C_{j}\left(t_{0}\right)$, which contradicts Lemma 4. Hence, (28) is true.

Now we proceed to prove (27). Its left hand side is

$$
\begin{aligned}
L H S & =W\left(\mathbf{G}_{-i, j}^{*}(t), G_{j}^{*}(t), \mathbf{v}\right)-W\left(\mathbf{G}_{-i, j}^{*}\left(t_{0}\right), G_{j}^{*}\left(t_{0}\right), \mathbf{v}\right) \\
& \geq W\left(\mathbf{G}_{-i, j}^{*}(t), G_{j}^{*}\left(t_{0}\right), \mathbf{v}\right)-W\left(\mathbf{G}_{-i, j}^{*}\left(t_{0}\right), G_{j}^{*}\left(t_{0}\right), \mathbf{v}\right)
\end{aligned}
$$

where the inequality comes from $t_{0} \leq t$. Equations (11) and (12) imply that $W\left(\mathbf{G}_{-i, j}^{*}(s), G_{j}^{*}(s), \mathbf{v}\right)=$ $W\left(\mathbf{G}_{-i, j}^{*}(s), \overline{\mathbf{v}}-\underline{\mathbf{v}}\right) G_{j}^{*}(s)+W\left(\mathbf{G}_{-i, j}^{*}(s), \underline{\mathbf{v}}\right)$. As a result,

$$
\begin{aligned}
& W\left(\mathbf{G}_{-i, j}^{*}(t), G_{j}^{*}\left(t_{0}\right), \mathbf{v}\right)-W\left(\mathbf{G}_{-i, j}^{*}\left(t_{0}\right), G_{j}^{*}\left(t_{0}\right), \mathbf{v}\right) \\
= & \left.W\left(\mathbf{G}_{-i, j}^{*}(s), \overline{\mathbf{v}}-\underline{\mathbf{v}}\right)\right|_{t_{0}} ^{t} G_{j}^{*}\left(t_{0}\right)+\left.W\left(\mathbf{G}_{-i, j}^{*}(s), \underline{\mathbf{v}}\right)\right|_{t_{0}} ^{t}
\end{aligned}
$$

Combining (29) and (30), we have

$$
L H S \geq\left. W\left(\mathbf{G}_{-i, j}^{*}(s), \overline{\mathbf{v}}-\underline{\mathbf{v}}\right)\right|_{t_{0}} ^{t} G_{j}^{*}\left(t_{0}\right)+\left.W\left(\mathbf{G}_{-i, j}^{*}(s), \underline{\mathbf{v}}\right)\right|_{t_{0}} ^{t}
$$

The definition of $t_{0}$ implies that $G_{i}^{*}(t)=G_{i}^{*}\left(t_{0}\right)$. Using this equation and the same argument for 
(31), we can rewrite the right hand side of (27) as

$$
R H S=\left.W\left(\mathbf{G}_{-i, j}^{*}(s), \overline{\mathbf{v}}-\underline{\mathbf{v}}\right)\right|_{t_{0}} ^{t} G_{i}^{*}\left(t_{0}\right)+\left.W\left(\mathbf{G}_{-i, j}^{*}(s), \underline{\mathbf{v}}\right)\right|_{t_{0}} ^{t}
$$

If we compare (31) and (32), it is straightforward to see that (28) implies (27).

Step III. This step shows

$$
W\left(\mathbf{G}_{-i}^{*}(t), \mathbf{v}\right)-C_{i}(t)>u_{i}^{*}
$$

Assumption A1 implies $C_{i}(t)-C_{i}\left(t_{0}\right)<C_{j}(t)-C_{j}\left(t_{0}\right)$. This inequality combined with (27) implies

$$
\left.\left[W\left(\mathbf{G}_{-i}^{*}(s), \mathbf{v}\right)-C_{i}(s)\right]\right|_{t_{0}} ^{t}>\left.\left[W\left(\mathbf{G}_{-j}^{*}(s), \mathbf{v}\right)-C_{j}(s)\right]\right|_{t_{0}} ^{t}
$$

Since $j \in \mathcal{A}^{*}(t)$, player $j$ 's payoff by choosing $t$ is $u_{j}^{*}$, which cannot be lower than that at score $t_{0}$. Therefore, $\left.\left[W\left(\mathbf{G}_{-j}^{*}(s), \mathbf{v}\right)-C_{j}(s)\right]\right|_{t_{0}} ^{t} \geq 0$, so (34) implies

$$
\left.\left[W\left(\mathbf{G}_{-i}^{*}(s), \mathbf{v}\right)-C_{i}(s)\right]\right|_{t_{0}} ^{t}>0
$$

Similarly, $i \in \mathcal{A}^{*}\left(t_{0}\right)$ implies that $W\left(\mathbf{G}_{-i}^{*}\left(t_{0}\right), \mathbf{v}\right)-C_{i}\left(t_{0}\right)=u_{i}^{*}$. Then, (35) implies (33).

In sum, (33) implies that player $i$ 's payoff by choosing $t$ is higher than his equilibrium payoff. This is a contradiction.

\section{Proof of Proposition 2 (Stochastic Dominance).}

Consider function $G_{j}^{*}(s)-G_{i}^{*}(s)$. Lemma 3 implies that $G_{j}^{*}(s)=G_{i}^{*}(s)$ for $s \geq \bar{s}_{j}^{*}$, so $G_{j}^{*}(s)-$ $G_{i}^{*}(s)=0$. For $s^{\prime}<\bar{s}_{j}^{*}$, Proposition 1 implies three possibilities: $i, j \notin \mathcal{A}^{*}\left(s^{\prime}\right) ; i \in \mathcal{A}^{*}\left(s^{\prime}\right), j \notin \mathcal{A}^{*}\left(s^{\prime}\right)$; or $i, j \in \mathcal{A}^{*}\left(s^{\prime}\right)$. In the first possibility, $G_{j}^{*}$ and $G_{i}^{*}$ remain constant in a neighborhood of $s^{\prime}$, therefore $G_{j}^{*}-G_{i}^{*}$ is non-increasing at $s^{\prime}$. In the second and third possibilities, $G_{j}^{*}(s)-G_{i}^{*}(s)$ is also nonincreasing in $s$ by the same argument in Step I of the proof for Proposition 1. Note that Proposition 1 relies on Assumptions A1 and A2. Therefore, $G_{j}^{*}(s)-G_{i}^{*}(s)$ is non-increasing for $s \geq 0$ and equals 0 for $s \geq \bar{s}_{j}^{*}$, so it cannot be negative. Hence, $G_{i}^{*}(s) \leq G_{j}^{*}(s)$ for $s \geq 0$.

Given $\mathcal{P} \subseteq \mathcal{N}$ and $\mathbf{v} \in[0, \infty)^{|\mathcal{P}|}$, define a vector function $\mathbf{W}_{\mathcal{P}}(\cdot, \mathbf{v}):[0,1]^{|\mathcal{P}|} \rightarrow[0, \infty)^{|\mathcal{P}|}$ that takes the form $\mathbf{W}_{\mathcal{P}}(\mathbf{x}, \mathbf{v})=\left(W\left(\mathbf{x}_{-k}, \mathbf{v}\right)\right)_{k \in \mathcal{P}}$ where $\mathbf{x}=\left(x_{j}\right)_{j \in \mathcal{P}}, \mathbf{x}_{-k}=\left(x_{j}\right)_{j \in \mathcal{P} \backslash\{k\}}$.

Proof of Proposition 3 (Ordered Densities). Consider any player $i$, and we first show that $G_{i}^{*}$ is differentiable at $s$. There are two possibilities: $i \in \mathcal{A}^{*}(s)$ or $i \notin \mathcal{A}^{*}(s)$.

First, suppose $i \notin \mathcal{A}^{*}(s)$. Since $s$ is assumed not to be on the boundary of the support of $G_{i}^{*}$, function $G_{i}^{*}$ is constant over $(s-\varepsilon, s+\varepsilon)$ for some $\varepsilon>0$. Therefore, $G_{i}^{*}$ is differentiable at $s$.

Second, suppose $i \in \mathcal{A}^{*}(s)$. Since $s$ is not on the support of any strategy's boundary, there exists $\varepsilon^{\prime}>0$ such that $\mathcal{A}^{*}\left(s^{\prime}\right)$ remains the same for $s^{\prime} \in\left(s-\varepsilon^{\prime}, s+\varepsilon^{\prime}\right)$. If player $k \in \mathcal{A}^{*}(s)$, then he is active over $\left(s-\varepsilon^{\prime}, s+\varepsilon^{\prime}\right)$. Therefore, his payoff at any score in the interval must be his equilibrium payoff. That is,

$$
W\left(\mathbf{G}_{-k}^{*}\left(s^{\prime}\right), \mathbf{v}\right)-C_{k}\left(s^{\prime}\right)=u_{k}^{*}
$$


where $\mathbf{G}_{-k}^{*}\left(s^{\prime}\right)=\left(G_{j}^{*}\left(s^{\prime}\right)\right)_{j \in \mathcal{P}^{*}(s) \backslash\{k\}}, \mathbf{v}=\left(v^{j}\right)_{j \in \mathcal{P}^{*}(s)}$ and $s^{\prime} \in\left(s-\varepsilon^{\prime}, s+\varepsilon^{\prime}\right)$. Notice that $G_{k}^{*}$ is constant over $\left(s-\varepsilon^{\prime}, s+\varepsilon^{\prime}\right)$ if $k \notin \mathcal{A}^{*}(s)$, so (36) for $k \in \mathcal{A}^{*}(s)$ is an equation system that implicitly defines $G_{k}^{*}$ for $j \in \mathcal{A}^{*}(s)$.

Consider matrix $\mathbf{J}_{\mathcal{A}^{*}(s)} \equiv\left(\partial W\left(\mathbf{G}_{-k}^{*}\left(s^{\prime}\right), \mathbf{v}\right) / \partial G_{j}\left(s^{\prime}\right)\right)_{k, j \in \mathcal{A}^{*}(s)}$. Under Assumption A2, $\mathbf{J}_{\mathcal{A}^{*}(s)}$ is invertible at $\mathbf{G}^{*}\left(s^{\prime}\right)=\left(G_{j}^{*}\left(s^{\prime}\right)\right)_{j \in \mathcal{A}^{*}(s)}$. Lemmas 10 and 13 in the supplementary note provide a proof for $\mathcal{A}^{*}(s)=\mathcal{P}^{*}(s)=\{1, \ldots, i\}$. The other cases can be proved similarly. Therefore, the Implicit Function Theorem implies that $G_{k}^{*}$ is differentiable at $s$ if $k \in \mathcal{A}^{*}(s)$. Since $i \in \mathcal{A}^{*}(s), G_{i}^{*}$ is differentiable at $s$.

Having shown that the derivatives exist, we proceed to show that they are ordered. Proposition 1 implies that there are three possible cases: $i, j \notin \mathcal{A}^{*}(s) ; i \in \mathcal{A}^{*}(s), j \notin \mathcal{A}^{*}(s)$; or $i, j \in \mathcal{A}^{*}(s)$. Note that Proposition 1 relies on Assumptions A1 and A2. We show below that $G_{j}^{* \prime}(s) \leq G_{i}^{* \prime}(s)$ in all three cases.

Consider the first two cases. Since $G_{j}^{* \prime}(s)=0$, we must have $G_{j}^{* \prime}(s) \leq G_{i}^{* \prime}(s)$.

Consider the third case. Since $i, j \in \mathcal{A}^{*}(s)$ in this case, we have (36) for $k=i, j$. Differentiating both sides of them with respect to $s^{\prime}$ and evaluating them at score $s$, we obtain

$$
\begin{aligned}
& \left.\frac{\partial W\left(\mathbf{G}_{-i}^{*}\left(s^{\prime}\right), \mathbf{v}\right)}{\partial G_{j}^{*}\left(s^{\prime}\right)}\right|_{s^{\prime}=s} G_{j}^{* \prime}(s)+\sum_{l \in \mathcal{A}^{*}(s) \backslash\{i, j\}}\left(\left.\frac{\partial W\left(\mathbf{G}_{-i}^{*}\left(s^{\prime}\right), \mathbf{v}\right)}{\partial G_{l}^{*}\left(s^{\prime}\right)}\right|_{s^{\prime}=s} G_{l}^{* \prime}(s)\right)=C_{i}^{\prime}(s) \\
& \left.\frac{\partial W\left(\mathbf{G}_{-j}^{*}\left(s^{\prime}\right), \mathbf{v}\right)}{\partial G_{i}^{*}\left(s^{\prime}\right)}\right|_{s^{\prime}=s} G_{i}^{* \prime}(s)+\sum_{l \in \mathcal{A}^{*}(s) \backslash\{i, j\}}\left(\left.\frac{\partial W\left(\mathbf{G}_{-j}^{*}\left(s^{\prime}\right), \mathbf{v}\right)}{\partial G_{l}^{*}\left(s^{\prime}\right)}\right|_{s^{\prime}=s} G_{l}^{* \prime}(s)\right)=C_{j}^{\prime}(s)
\end{aligned}
$$

Notice that

$$
\begin{aligned}
\left.\frac{\partial W\left(\mathbf{G}_{-i}^{*}\left(s^{\prime}\right), \mathbf{v}\right)}{\partial G_{l}^{*}\left(s^{\prime}\right)}\right|_{s^{\prime}=s} & =W\left(\mathbf{G}_{-i, l}^{*}(s), \overline{\mathbf{v}}-\underline{\mathbf{v}}\right) \\
& \geq W\left(\mathbf{G}_{-j, l}^{*}(s), \overline{\mathbf{v}}-\underline{\mathbf{v}}\right) \\
& =\left.\frac{\partial W\left(\mathbf{G}_{-j}^{*}\left(s^{\prime}\right), \mathbf{v}\right)}{\partial G_{l}^{*}\left(s^{\prime}\right)}\right|_{s^{\prime}=s}
\end{aligned}
$$

where the equalities come from (12) and the inequality comes from Proposition 2. Inequality (39) implies that the second term in (37) is no smaller than that in (38). In addition, Assumption A1 implies that the right hand side of (37) is smaller than that of (38). Therefore, the first term in (37) must be smaller than that in (38). That is,

$$
\left.\frac{\partial W\left(\mathbf{G}_{-i}^{*}\left(s^{\prime}\right), \mathbf{v}\right)}{\partial G_{j}^{*}\left(s^{\prime}\right)}\right|_{s^{\prime}=s} G_{j}^{* \prime}(s)<\left.\frac{\partial W\left(\mathbf{G}_{-j}^{*}\left(s^{\prime}\right), \mathbf{v}\right)}{\partial G_{i}^{*}\left(s^{\prime}\right)}\right|_{s^{\prime}=s} G_{i}^{* \prime}(s)
$$

Similar to (39), we have

$$
\left.\frac{\partial W\left(\mathbf{G}_{-i}^{*}\left(s^{\prime}\right), \mathbf{v}\right)}{\partial G_{j}^{*}\left(s^{\prime}\right)}\right|_{s^{\prime}=s}=\left.\frac{\partial W\left(\mathbf{G}_{-j}^{*}\left(s^{\prime}\right), \mathbf{v}\right)}{\partial G_{i}^{*}\left(s^{\prime}\right)}\right|_{s^{\prime}=s}=W\left(\mathbf{G}_{-i, j}^{*}(s), \overline{\mathbf{v}}-\underline{\mathbf{v}}\right)
$$


which is nonnegative. Since $i, j \in \mathcal{A}^{*}(s)$, the definition of $\mathcal{A}^{*}(s)$ implies that $G_{j}^{* \prime}(s), G_{i}^{* \prime}(s)>0$. Therefore, (40) implies that $W\left(\mathbf{G}_{-i, j}^{*}(s), \overline{\mathbf{v}}-\underline{\mathbf{v}}\right) \neq 0$, then it also implies $G_{j}^{* \prime}(s)<G_{i}^{* \prime}(s)$.

In the remainder of Appendix A, we first introduce in Lemma 5 a property of equation system (4). Then, using the property, we verify the claims in the definition of pseudo strategies and prove Propositions 4 and 5. For $i \in \mathcal{P}(s)$, equation system (4) for $j \in \mathcal{P}(s) \backslash\{i\}$ implicitly defines $\left(G_{j}(s)\right)_{j \in \mathcal{P}(s) \backslash\{i\}}$ as a function of $G_{i}(s)$. Let $i^{\prime}$ be the weakest player in $\mathcal{P}(s) \backslash\{i\}$, Lemma 5 shows that $G_{i^{\prime}}(s)$ is decreasing in $G_{i}(s)$.

Lemma 5 Given $s$ and $G_{i}(s)$, suppose that $\left(G_{j}(s)\right)_{j \in \mathcal{P} \backslash\{i\}}$ in $[0,1]^{|\mathcal{P}|-1}$ solves (4) for $j \in \mathcal{P} \backslash\{i\}$ where $\mathcal{P}$ is a subset of $\mathcal{N}$ and $i \in \mathcal{P}$. Let $i^{\prime}$ be the weakest player in $\mathcal{P}(s) \backslash\{i\}$, then $G_{i^{\prime}}(s)$ increases if $G_{i}(s)$ decreases.

Proof. Suppose $\mathcal{P}=\{1, \ldots, i\}$, and the other cases can be proved similarly. Differentiating both sides of (4) with respect to $G_{i}(s)$, we have

$$
\sum_{l \in \mathcal{P} \backslash\{i, j\}}\left(\frac{\partial W_{\mathcal{P}}\left(\mathbf{G}_{-j}(s), \mathbf{v}\right)}{\partial G_{l}(s)} \frac{\partial G_{l}(s)}{\partial G_{i}(s)}\right)=-\frac{\partial W_{\mathcal{P}}\left(\mathbf{G}_{-j}(s), \mathbf{v}\right)}{\partial G_{i}(s)}
$$

for $j \in \mathcal{P} \backslash\{i\}$. Let $\mathbf{J}_{\mathcal{P}}$ be the Jacobian matrix of vector function $\mathbf{W}_{\mathcal{P}}(\cdot, \mathbf{v})$, so $\mathbf{J}_{\mathcal{P}}$ is a $|\mathcal{P}| \times|\mathcal{P}|$ matrix. Let $\mathbf{J}_{\mathcal{P} \backslash\{i\}}$ be $\mathbf{J}_{\mathcal{P}}$ without row $i$ and column $i$, so $\mathbf{J}_{\mathcal{P} \backslash\{i\}}$ is a $(|\mathcal{P}|-1) \times(|\mathcal{P}|-1)$ matrix. In addition, let $\boldsymbol{\delta}$ and $\mathbf{d}$ be vectors of $i-1$ rows, where row $j$ of $\boldsymbol{\delta}$ is $\partial G_{j}(s) / \partial G_{i}(s)$ and row $j$ of $\mathbf{d}$ is $\partial W_{\mathcal{P}}\left(\mathbf{G}_{-j}(s), \mathbf{v}\right) / \partial G_{i}(s)$ for $j \in \mathcal{P} \backslash\{i\}$. We can rewrite (41) in matrix form

$$
\mathbf{J}_{\mathcal{P} \backslash\{i\}} \boldsymbol{\delta}=-\mathbf{d}
$$

Given $\mathcal{P}=\{1, \ldots, i\}$, Lemmas 10 and 13 in the supplementary note imply that $\operatorname{det} \mathbf{J}_{\mathcal{P}} \neq 0$ and $\operatorname{det} \mathbf{J}_{\mathcal{P} \backslash\{i\}} \neq 0$. Therefore, the Implicit Function Theorem implies that (4) for $j \in \mathcal{P} \backslash\{i\}$ implicitly defines $\left(G_{j}(s)\right)_{j \in \mathcal{P} \backslash\{i\}}$ as a function of $G_{i}(s)$. Moreover, the function is differentiable with derivatives $\boldsymbol{\delta}$ satisfying (42). Since $\operatorname{det} \mathbf{J}_{\mathcal{P} \backslash\{i\}} \neq 0$, we can solve (42) for $\boldsymbol{\delta}$. The $j$ th entry of $\boldsymbol{\delta}$ is

$$
\delta_{j}=\operatorname{det} \tilde{\mathbf{J}}_{\mathcal{P} \backslash\{i\}, j} / \operatorname{det} \mathbf{J}_{\mathcal{P} \backslash\{i\}}
$$

where $\tilde{\mathbf{J}}_{\mathcal{P} \backslash\{i\}, j}$ is $\mathbf{J}_{\mathcal{P} \backslash\{i\}}$ with its $j$ th column replaced by $\mathbf{d}$.

Consider GPS first. Lemma 13 in the supplementary note discusses the sign of $\operatorname{det} \mathbf{J}_{\mathcal{P} \backslash\{i\}}$, and Lemma 15 in it discusses the sign of $\operatorname{det} \tilde{\mathbf{J}}_{\mathcal{P} \backslash\{i\}, j}$. They imply that the two determinants have opposite signs. Therefore, (43) implies that $\delta_{j}<0$.

Consider QPS. Lemma 10 in the supplementary note shows that the sign of $\operatorname{det} \mathbf{J}_{\mathcal{P} \backslash\{i\}}$ is $(-1)^{i}$. In order to show $\delta_{i}<0$, we need to prove $\operatorname{det} \tilde{\mathbf{J}}_{\mathcal{P} \backslash\{i\}, i^{\prime}}$ has the same sign with $\operatorname{det} \mathbf{J}_{\mathcal{P} \backslash\{i\}}$. Lemma 16 in the supplementary note shows that $\operatorname{det} \tilde{\mathbf{J}}_{\mathcal{P} \backslash\{i\}, j}-\operatorname{det} \tilde{\mathbf{J}}_{\mathcal{P} \backslash\{i\}, j-1}$ has sign $(-1)^{i}$ for all $j \in \mathcal{P} \backslash\{i\}$. Therefore, (43) implies $\delta_{1}>\delta_{2}>\ldots>\delta_{j-1}$. Suppose $\delta_{j-1} \geq 0$, then $\delta_{1}, \ldots, \delta_{j-2}>0$, so $\mathbf{J}_{\mathcal{P} \backslash\{i\}} \boldsymbol{\delta} \gg 0$, which contradicts with (42). Therefore, $\delta_{j-1}<0$. 
Lemma 6 Suppose $2 \leq i \leq m+1$ and $u_{1}^{*}, \ldots, u_{i}^{*}$ are players $1, \ldots, i$ 's payoffs in an equilibrium. Then, the pseudo strategies $\hat{G}_{1}, \ldots, \hat{G}_{i}$ yielding $u_{1}^{*}, \ldots, u_{i}^{*}$ are well-defined.

Proof. We first prove for the case of $i=m+1$, then extend the proof to $i<m+1$.

Suppose $i=m+1$. Denote the strategy of player $i$ in the equilibrium as $G_{i}^{*}$. Because there is no aggregate gap, at least two players' equilibrium strategies have score 0 as the lower boundary of their supports. Proposition 2 implies that $m$ and $m+1$ are among such players. Note that Proposition 2 relies on Assumptions A1 and A2. Therefore, if $i=m+1$, score $\underline{s}_{i}$ in the definition of pseudo strategies equals 0. Moreover, equation system (4) for $j \in \hat{\mathcal{P}}\left(\underline{s}_{i}\right)$ and $s=\underline{s}_{i}$ has a unique solution $\left(\hat{G}_{j}\left(\underline{s}_{i}\right)\right)_{j \in \hat{\mathcal{P}}\left(\underline{s}_{i}\right)}$ in $[0,1]^{\left|\hat{\mathcal{P}}\left(\underline{s}_{i}\right)\right|}$, and it is

$$
\begin{aligned}
\hat{G}_{m}\left(\underline{s}_{i}\right) & =G_{m}^{*}(0) \\
\hat{G}_{m+1}\left(\underline{s}_{i}\right) & =G_{m+1}^{*}(0)
\end{aligned}
$$

Having defined $\left(\hat{G}_{j}\right)_{j \in \hat{\mathcal{P}}\left(\underline{s}_{i}\right)}$ at $\underline{s}_{i}$, we next extend the definition to scores above $\underline{s}_{i}$. Differentiating both sides of (4) with respect to $s$, we have

$$
\mathbf{J}_{\hat{\mathcal{P}}\left(\underline{s}_{i}\right)} \mathbf{g}=\mathbf{c}
$$

where $\mathbf{g}=\left(G_{j}^{\prime}(s)\right)_{j \in \hat{\mathcal{P}}\left(\underline{s}_{i}\right)}, \mathbf{c}=\left(C_{j}^{\prime}(s)\right)_{j \in \hat{\mathcal{P}}\left(\underline{s}_{i}\right)}$ and $\mathbf{J}_{\hat{\mathcal{P}}\left(\underline{s}_{i}\right)}$ is the Jacobian matrix of the vector function $\mathbf{W}_{\hat{\mathcal{P}}\left(\underline{s}_{i}\right)}(\cdot, \mathbf{v})$. Similar to $(42)$, we can verify that $\operatorname{det} \mathbf{J}_{\hat{\mathcal{P}}_{\left(s_{i}\right)}} \neq 0$ under Assumption A1, and rewrite (46) as

$$
\mathbf{g}=\left(\mathbf{J}_{\hat{\mathcal{P}}\left(\underline{s}_{i}\right)}\right)^{-1} \mathbf{c}
$$

Then, Theorem 20.10 of Olver (2007) implies that, there exists $\varepsilon>0$ such that the ordinary differential equation system (47) with initial conditions (44) and (45) has a unique solution for $s \in\left[\underline{s}_{i}, \underline{s}_{i}+\varepsilon\right)$.

Consider the claim that there exists $\bar{s}_{i}>\underline{s}_{i}$ such that $\hat{G}_{i}\left(\bar{s}_{i}\right)=1$. Since $\lim _{s \rightarrow \infty} C_{j}(s)=\infty$ and the values of $\mathbf{W}_{\hat{\mathcal{P}}\left(\underline{s}_{i}\right)}$ are bounded above by $v^{1}$, the solution to (47) cannot be extended to $\left[\underline{s}_{i},+\infty\right)$. Theorem 20.10 of Olver (2007) implies that the solution to (47) extends to higher scores until the solution reaches the boundary of the domain of $\mathbf{W}_{\hat{\mathcal{P}}\left(\underline{s}_{i}\right)}(\cdot, \mathbf{v})$. Therefore, it extends until $\hat{G}_{j}(s)=0$ or $\hat{G}_{j}(s)=1$ for some $j \in \hat{\mathcal{P}}\left(\underline{s}_{i}\right)$. Let us show that $\hat{G}_{j}(s)>0$ for $s>\underline{s}_{i}$. By the same argument for (42), the $j$ th entry of $\mathbf{g}$ is

$$
G_{j}^{\prime}(s)=-\operatorname{det} \tilde{\mathbf{J}}_{\hat{\mathcal{P}}\left(\underline{s}_{i}\right), j} / \operatorname{det} \mathbf{J}_{\hat{\mathcal{P}}\left(\underline{s}_{i}\right)}
$$

where $\tilde{\mathbf{J}}_{\hat{\mathcal{P}}\left(\underline{s}_{i}\right), j}$ is $\mathbf{J}_{\hat{\mathcal{P}}\left(\underline{s}_{i}\right)}$ with its $j$ th column replaced by c. By the same argument for $(43), \hat{G}_{j}^{\prime}(s)>0$ because the two determinants in (48) have opposite signs. Hence, $\hat{G}_{j}(s)>0$ for $s>\underline{s}_{i}$. As a result, we verify that there exists $\bar{s}_{l} \geq \underline{s}_{i}$ such that $\hat{G}_{l}\left(\bar{s}_{l}\right)=1$ for some $l \in \hat{\mathcal{P}}\left(\underline{s}_{i}\right)$. In addition, by a similar argument to Step I in the proof of Proposition $1, \hat{G}_{i}(s) \geq \hat{G}_{i-1}(s)$ for $s \geq \underline{s}_{i}$. Therefore, when $\hat{G}_{i}$ and $\hat{G}_{i-1}$ are extended towards higher scores, $\hat{G}_{i}$ reaches 1 before $\hat{G}_{i-1}$ does. Hence, there exists $\bar{s}_{i}>\underline{s}_{i}$ such that $\hat{G}_{i}\left(\bar{s}_{i}\right)=1$. Note that Assumption A1 and A2 are important because Proposition 1 relies on the assumptions. 
We have verified the claims in the first step of the definition if $i=m+1$. Let us move to the second step of the definition. Consider the claim that there exists $l^{\prime} \notin \hat{\mathcal{P}}\left(\underline{s}_{i}\right)$ such that (4) holds for some score $\underline{s}_{l^{\prime}} \geq \underline{s}_{i}$. Consider the minimum score $\underline{s}_{m-1}^{*}$ in the support of equilibrium strategies $G_{m-1}^{*}$ of $m-1$, who is the weakest player outside of $\hat{\mathcal{P}}\left(\underline{s}_{i}\right)$. Proposition 1 implies that $\mathcal{A}^{*}\left(\underline{s}_{m-1}^{*}\right)=\hat{\mathcal{P}}\left(\underline{s}_{i}\right) \cup\{m-1\}$, so the indifference condition (4) holds for all players in $\mathcal{A}^{*}\left(\underline{s}_{m-1}^{*}\right)$. Proposition 2 implies that $\underline{s}_{m-1}^{*}>\underline{s}_{i}$. Hence, we verify that $m-1 \notin \hat{\mathcal{P}}\left(\underline{s}_{i}\right)$ and (4) holds for the score $\underline{s}_{m-1}^{*} \geq \underline{s}_{i}$.

The third step repeats the first two steps for higher scores, and the arguments are the same as above. Therefore, we prove Lemma 6 for $i=m+1$.

Now, suppose $i=m$. Since there is no aggregate gap, we have $m-1$ and $m \in \mathcal{A}^{*}\left(\bar{s}_{m+1}^{*}\right)$. The indifference condition for $m$ is $W\left(\mathbf{G}_{-m}^{*}\left(\bar{s}_{m+1}^{*}\right), \mathbf{v}\right)-C_{m}\left(\bar{s}_{m+1}^{*}\right)=u_{m}^{*}$. If we increase $G_{j}^{*}\left(\bar{s}_{m+1}^{*}\right)$ to 1 for $j \neq m$, the indifference condition implies

$$
W_{\{m, m-1\}}\left(G_{m-1}^{*}\left(\bar{s}_{m+1}^{*}\right), \mathbf{v}\right)-C_{m}\left(\bar{s}_{m+1}^{*}\right) \geq u_{m}^{*}
$$

Therefore, the definition of $\underline{s}_{i}$ implies $\underline{s}_{i} \leq \bar{s}_{m+1}^{*}$. Then, we have

$$
W_{\{m, m-1\}}\left(G_{m}^{*}\left(\bar{s}_{m+1}^{*}\right), \mathbf{v}\right)-C_{m-1}\left(\underline{s}_{i}\right) \geq W_{\{m, m-1\}}\left(G_{m}^{*}\left(\bar{s}_{m+1}^{*}\right), \mathbf{v}\right)-C_{m-1}\left(\bar{s}_{m+1}^{*}\right) \geq u_{m-1}^{*}
$$

where the second inequality comes from a similar argument for (49). Recall that the definition of $\hat{G}_{m}$ requires

$$
W_{\{m, m-1\}}\left(\hat{G}_{m}\left(\underline{s}_{i}\right), \mathbf{v}\right)-C_{m-1}\left(\underline{s}_{i}\right)=u_{m-1}^{*}
$$

so (50) implies $\hat{G}_{m}\left(\underline{s}_{i}\right) \leq G_{m}^{*}\left(\bar{s}_{m+1}^{*}\right) \leq 1$. Hence, we prove that equation system (4) for $j \in \hat{\mathcal{P}}\left(\underline{s}_{i}\right)$ and $s=\underline{s}_{i}$ has a unique solution $\left(\hat{G}_{j}\left(\underline{s}_{i}\right)\right)_{j \in \hat{\mathcal{P}}\left(\underline{s}_{i}\right)}$ in $[0,1]^{\mid \hat{\mathcal{P}}}\left(\underline{s}_{i}\right) \mid$. Then, following the same arguments below (45), we can verify that the pseudo strategies are well-defined for the case of $i=m$. Moreover, we can proceed in the same fashion to even smaller values $i=m-1, \ldots, 2$.

Lemma 7 Given any equilibrium, suppose player $i$ deviates to $s \in\left[\underline{s}_{i+1}^{*}, \underline{s}_{i}^{*}\right)$, then his expected payoff is lower than $u_{i}^{*}$.

Proof. The definition of equilibrium implies that player $i$ 's expected payoff is no higher than $u_{i}^{*}$ if he deviates to $s \in\left[\underline{s}_{i+1}^{*}, \underline{s}_{i}^{*}\right)$. As a result, it is sufficient to show that $i$ 's expected payoff cannot be $u_{i}^{*}$ if he deviates to $s$. Suppose there is $s^{\prime} \in\left[\underline{s}_{i+1}^{*}, \underline{s}_{i}^{*}\right)$ such that

$$
W\left(\hat{\mathbf{G}}_{-i}\left(s^{\prime}\right), \mathbf{v}\right)-C_{i}\left(s^{\prime}\right)=W\left(\mathbf{G}_{-i}^{*}\left(\underline{s}_{i}^{*}\right), \mathbf{v}\right)-C_{i}\left(\underline{s}_{i}^{*}\right)=u_{i}^{*}
$$

Then, the lemma is violated. We can rewrite equation (51) as

$$
W\left(\mathbf{G}_{-i}^{*}\left(\underline{s}_{i}^{*}\right), \mathbf{v}\right)-W\left(\mathbf{G}_{-i}^{*}\left(s^{\prime}\right), \mathbf{v}\right)=C_{i}\left(\underline{s}_{i}^{*}\right)-C_{i}\left(s^{\prime}\right)
$$

Since there is no aggregate gap and Proposition 1, players $i, i+1 \in \mathcal{A}^{*}\left(\underline{s}_{i}^{*}\right)$ and $i+1, i+2 \in \mathcal{A}^{*}\left(s^{\prime}\right)$. 
Since $i+1$ is active at $\underline{s}_{i}^{*}$ and $s^{\prime}$, he must receive the same payoff by choosing either score. That is,

$$
W\left(\mathbf{G}_{-(i+1)}^{*}\left(s^{\prime}\right), \mathbf{v}\right)-C_{i+1}\left(s^{\prime}\right)=W\left(\mathbf{G}_{-(i+1)}^{*}\left(\underline{s}_{i}^{*}\right), \mathbf{v}\right)-C_{i}\left(\underline{s}_{i}^{*}\right)
$$

which can be rewritten as

$$
W\left(\mathbf{G}_{-(i+1)}^{*}\left(\underline{s}_{i}^{*}\right), \mathbf{v}\right)-W\left(\mathbf{G}_{-(i+1)}^{*}\left(s^{\prime}\right), \mathbf{v}\right)=C_{i+1}\left(\underline{s}_{i}^{*}\right)-C_{i+1}\left(s^{\prime}\right)
$$

Assumption A1 implies that the right hand side of (52) is smaller than that of (53). Therefore,

$$
W\left(\mathbf{G}_{-i}^{*}\left(\underline{s}_{i}^{*}\right), \mathbf{v}\right)-W\left(\mathbf{G}_{-i}^{*}\left(s^{\prime}\right), \mathbf{v}\right)<W\left(\mathbf{G}_{-(i+1)}^{*}\left(\underline{s}_{i}^{*}\right), \mathbf{v}\right)-W\left(\mathbf{G}_{-(i+1)}^{*}\left(s^{\prime}\right), \mathbf{v}\right)
$$

which can be rewritten as

$$
\begin{aligned}
& W\left(\mathbf{G}_{-i, i+1}^{*}\left(\underline{s}_{i}^{*}\right), G_{i+1}^{*}\left(\underline{s}_{i}^{*}\right), \mathbf{v}\right)-W\left(\mathbf{G}_{-i, i+1}^{*}\left(\underline{s}_{i}^{*}\right), G_{i+1}^{*}\left(s^{\prime}\right), \mathbf{v}\right) \\
& +W\left(\mathbf{G}_{-i, i+1}^{*}\left(s^{\prime}\right), G_{i+1}^{*}\left(\underline{s}_{i}^{*}\right), \mathbf{v}\right)-W\left(\mathbf{G}_{-i, i+1}^{*}\left(s^{\prime}\right), G_{i+1}^{*}\left(s^{\prime}\right), \mathbf{v}\right) \\
< & W\left(\mathbf{G}_{-i, i+1}^{*}\left(\underline{s}_{i}^{*}\right), G_{i}^{*}\left(\underline{s}_{i}^{*}\right), \mathbf{v}\right)-W\left(\mathbf{G}_{-i, i+1}^{*}\left(\underline{s}_{i}^{*}\right), G_{i}^{*}\left(s^{\prime}\right), \mathbf{v}\right) \\
& +W\left(\mathbf{G}_{-i, i+1}^{*}\left(s^{\prime}\right), G_{i}^{*}\left(\underline{s}_{i}^{*}\right), \mathbf{v}\right)-W\left(\mathbf{G}_{-i, i+1}^{*}\left(s^{\prime}\right), G_{i}^{*}\left(s^{\prime}\right), \mathbf{v}\right)
\end{aligned}
$$

Then, by the same argument after (37) and (38), the inequality above implies

$$
G_{i}^{*}\left(\underline{s}_{i}^{*}\right)-G_{i}^{*}\left(s^{\prime}\right)>G_{i+1}^{*}\left(\underline{s}_{i}^{*}\right)-G_{i+1}^{*}\left(s^{\prime}\right)
$$

Since $s^{\prime}<\underline{s}_{i}^{*}$, function $G_{i}^{*}$ remains at 0 , so $G_{i}^{*}\left(\underline{s}_{i}^{*}\right)-G_{i}^{*}\left(s^{\prime}\right)=0$. Moreover, player $i+1$ is active at $s^{\prime}$, so $G_{i+1}^{*}\left(\underline{s}_{i}^{*}\right)-G_{i+1}^{*}\left(s^{\prime}\right)>0$. Hence, $G_{i}^{*}\left(\underline{s}_{i}^{*}\right)-G_{i}^{*}\left(s^{\prime}\right)<G_{i+1}^{*}\left(\underline{s}_{i}^{*}\right)-G_{i+1}^{*}\left(s^{\prime}\right)$, which contradicts $(55)$.

Proof of Proposition 4 (Dent vs Gap). We first prove for the case of $i=m+1$. Let $\hat{G}_{1}, \ldots, \hat{G}_{m+1}$ be the pseudo strategies yielding the payoffs $u_{1}^{*}, \ldots, u_{m+1}^{*}$.

Suppose that $\left(s^{\prime}, s^{\prime \prime}\right)$ is the first gap in the support of $G_{m+1}^{*}$ if we move from score 0 to higher scores. Let us show that $\hat{G}_{m+1}$ has a dent over $\left(s^{\prime}, s^{\prime \prime}\right)$.

Because of Lemma 7 , the construction of pseudo strategies implies that $\hat{\mathcal{P}}(s)=\mathcal{P}^{*}(s)$ for $s \in\left(s, s^{\prime}\right]$. Therefore, $\hat{G}_{m+1}\left(s^{\prime}\right)=G_{m+1}^{*}\left(s^{\prime}\right)$. Moreover, Lemma 5 implies that $\hat{G}_{m}(s)<G_{m}^{*}(s)$ for $s \in\left(s^{\prime}, \underline{s}_{m}^{*}\right]$ and $j \in \hat{\mathcal{P}}(s) \backslash\{m+1\}$. At $\underline{s}_{m}^{*}$, we have

$$
W\left(\hat{\mathbf{G}}_{-m}\left(\underline{s}_{m}^{*}\right), \mathbf{v}\right)-C_{m}\left(\underline{s}_{m}^{*}\right) \leq W\left(\mathbf{G}_{-m}^{*}\left(\underline{s}_{m}^{*}\right), \mathbf{v}\right)-C_{m}\left(\underline{s}_{m}^{*}\right)=u_{m}^{*}
$$

where the inequality comes from Lemmas 11 and 14 in the supplementary note. Then, the definition of $\underline{s}_{m}$ implies $\underline{s}_{m} \geq \underline{s}_{m}^{*}$. Similarly, we can prove that $\hat{G}_{j}(s)<G_{j}^{*}(s)$ for $s \in\left[\underline{s}_{m}^{*}, \underline{s}_{m-1}^{*}\right]$ and $\underline{s}_{m-1}>\underline{s}_{m-1}^{*}$.

Let us show that $\hat{\mathcal{P}}\left(s^{\prime \prime}\right)=\mathcal{P}^{*}\left(s^{\prime \prime}\right)$. Suppose otherwise, we have $\left(\hat{G}_{j}(s)\right)_{j \in \mathcal{P}^{*}\left(s^{\prime \prime}\right)}$ and it can be extended to lower scores. Consider $\hat{G}_{j^{\prime}}$ for the strongest player in $\mathcal{P}^{*}\left(s^{\prime \prime}\right)$. Since $\underline{s}_{j^{\prime}}^{*}>\underline{s}_{j}, \hat{G}_{j^{\prime}}$ must 
reach 0 at a score in $\left(\underline{s}_{j^{\prime}}^{*}, s^{\prime \prime}\right)$. The score, by definition, is $\underline{s}_{j}$.

So far, we have shown that $\hat{G}_{m+1}\left(s^{\prime}\right)=G_{m+1}^{*}\left(s^{\prime}\right), \hat{G}_{m+1}\left(s^{\prime \prime}\right)=G_{m+1}^{*}\left(s^{\prime \prime}\right)$ and $\hat{G}_{m+1}(s) \leq$ $G_{m+1}^{*}(s)$ for $s \in\left(s^{\prime}, s^{\prime \prime}\right)$. Therefore, $\hat{G}_{m+1}$ has a dent over $\left(s^{\prime}, s^{\prime \prime}\right)$. Similarly, we can show that $\hat{G}_{m+1}$ has a dent at higher gaps of $G_{m+1}^{*}$ as well.

Suppose that $\left(s^{\prime}, s^{\prime \prime}\right)$ is the first dent of $\hat{G}_{m+1}$ if we move from score 0 to higher scores. Let us show that $G_{m+1}^{*}$ has a gap over $\left(s^{\prime}, s^{\prime \prime}\right)$.

By the above argument, we have $\hat{G}_{m+1}\left(s^{\prime}\right)=G_{m+1}^{*}\left(s^{\prime}\right)$. Then, $G_{m+1}^{*}(s)=G_{m+1}^{*}\left(s^{\prime}\right)$ for $s \in$ $\left[s^{\prime}, s^{\prime}+\varepsilon\right)$ for some $\varepsilon>0$, otherwise system (4) for $j \in \mathcal{P}^{*}\left(s^{\prime}\right)$ and $s \in\left(s^{\prime}, s^{\prime}+\varepsilon\right)$ has two solutions, which is a contradiction. Therefore, there is a gap with lower bound $s^{\prime}$ in the support of $G_{m+1}^{*}$. Now we prove that the upper bound is $s^{\prime \prime}$. Suppose the upper bound is $s^{\prime \prime \prime}$ and $s^{\prime \prime \prime}<s^{\prime \prime}$, then the above argument implies that $\hat{G}_{m+1}$ has a dent over $\left(s^{\prime}, s^{\prime \prime \prime}\right)$. Then, $\hat{G}_{m+1}$ cannot have a dent $\left(s^{\prime}, s^{\prime \prime}\right)$, which is a contradiction. Suppose the upper bound $s^{\prime \prime \prime}>s^{\prime \prime}$, then $\hat{G}_{m+1}$ has a dent $\left(s^{\prime}, s^{\prime \prime \prime}\right)$. This is also a contradiction. Hence, $\hat{G}_{m+1}$ has a gap over $\left(s^{\prime}, s^{\prime \prime}\right)$.

Similarly, we can show that $G_{m+1}^{*}$ has a gap over other dents of $\hat{G}_{m+1}$ as well. As a result, Proposition 4 is proved for $i=m+1$. The proof for $i<m+1$ is similar.

Lemma 8 Given any equilibrium, each player's equilibrium strategy $G_{i}^{*}$ is absolutely continuous. Moreover, the support of $G_{i}^{*}$ has at most a finite number of gaps.

Proof. We first show that $G_{i}^{*}$ has at most a finite number of gaps. Let $\hat{G}_{1}, \ldots, \hat{G}_{i}$ be the pseudo strategies yielding equilibrium payoffs $u_{1}^{*}, \ldots, u_{i}^{*}$. By the definition of pseudo strategies, there are finite number of intervals such that i) each interval is closed at the lower boundary and open at the upper boundary, and ii) the set of participating player $\mathcal{P}$ remains the same over each interval. Pick any of these intervals, denote it as $\left[s_{0}, s_{0}^{\prime}\right)$. The set of participating players $\mathcal{P}(s)=\mathcal{P}\left(s_{0}\right)$ for $s \in\left[s_{0}, s_{0}^{\prime}\right)$. Let $\hat{\mathbf{G}}=\left(\hat{G}_{i}\right)_{i \in \mathcal{P}\left(s_{0}\right)}$ and $\mathbf{c}=\left(c_{i}\right)_{i \in \mathcal{P}\left(s_{0}\right)}$. Then, similar to (47), the pseudo strategies $\hat{\mathbf{G}}$ is differentiable, and it solves ordinary differential equation system

$$
\hat{\mathbf{G}}^{\prime}=\left(\mathbf{J}_{\mathcal{P}\left(s_{0}\right)}\right)^{-1} \mathbf{c}
$$

with initial condition $\hat{\mathbf{G}}\left(s_{0}\right)=\left(\hat{G}_{i}\left(s_{0}\right)\right)_{i \in \mathcal{P}\left(s_{0}\right)}$.

The right hand side of (56) is an analytic function of $\hat{\mathbf{G}}$. In addition, Theorem 20.10 of Olver (2007) implies that the solution $\hat{\mathbf{G}}$ is analytic in a small neighborhood of $s_{0}$. Therefore, the right hand side is a composition of analytic functions, hence $\hat{\mathbf{G}}^{\prime}$ is also analytic in the neighborhood. The same argument applies to other points in $\left[s_{0}, s_{0}^{\prime}\right)$, so both $\hat{G}_{i}$ and $\hat{G}_{i}^{\prime}$ are analytic over the interval.

Given that $\hat{G}_{i}^{\prime}$ is analytic over $\left[s_{0}, s_{0}^{\prime}\right.$ ), the Identity Theorem ${ }^{30}$ implies that $\hat{G}_{i}^{\prime}$ either has a finite number of roots in its domain or $\hat{G}_{i}^{\prime}=0$. Either case implies that $\hat{G}_{i}$ has at most a finite number of dents. Then, Proposition 4 implies that the support of $G_{i}^{*}$ has at most a finite number of gaps.

We prove the absolute continuity in three steps. First, $G_{i}^{*}$ is differentiable except at a finite number of scores. Because each player's equilibrium strategy has at most a finite number of gaps, there are at most a finite number of scores that are on the boundary of an equilibrium strategy's

\footnotetext{
${ }^{30}$ See Pugh (2002), p. 256.
} 
support. Then, Proposition 3 implies that $G_{i}^{*}$ is differentiable except at a finite number of scores. Second, the derivatives of the equilibrium strategies are uniformly bounded. To see why, suppose $G_{i}^{* \prime}$ is not uniformly bounded, then there is a sequence of scores $\left\{s^{k}\right\}_{k=1}^{\infty}$ converging to $s^{\prime \prime}$ such that $\lim _{k \rightarrow \infty} G_{i}^{* \prime}\left(s^{k}\right)=+\infty$. However, this would imply that the left hand side of (37) evaluated at $s^{\prime \prime}$ goes to $+\infty$. This is a contradiction. Third, the two steps above and the continuity of $G_{i}^{*}$ ensured by Lemma 2 imply that $G_{i}^{*}$ is Lipschitz continuous, which is stronger than absolute continuity.

Proof of Proposition 5. We prove for $i=m$, and the proof for $i<m$ is similar. The proof has two parts.

Part I. Player $m+1$ 's payoff at $\bar{s}_{m+1}^{*}$ against $\hat{G}_{1}, \ldots, \hat{G}_{m}$ is $u_{m+1}^{*}$. To see why, notice that the definition of pseudo strategies implies that $\hat{G}_{i}\left(\bar{s}_{m+1}^{*}\right)=G_{i}^{*}\left(\bar{s}_{m+1}^{*}\right)$ for $i=1, \ldots, m$, where $\left(\hat{G}_{i}\right)_{i \in\{1, \ldots, m\}}$ are the pseudo strategies yielding payoffs $u_{1}^{*}, \ldots, u_{m}^{*}$. Therefore, $\bar{s}_{m+1}^{*} \in \hat{S}_{m}$ and $\bar{s}_{m+1}^{*}$ is the highest score in $\hat{S}_{m}$. Moreover, $\hat{G}_{i}\left(\bar{s}_{m+1}^{*}\right)=G_{i}^{*}\left(\bar{s}_{m+1}^{*}\right)$ implies that player $m+1$ 's payoff at $\bar{s}_{m+1}^{*}$ against $\hat{G}_{1}, \ldots, \hat{G}_{m}$ is $u_{m+1}^{*}$.

Part II. Any $s \in \hat{S}_{m}$ cannot give player $m+1$ a payoff higher than $u_{m+1}^{*}$ when others choose $\hat{G}_{i}$. We prove this claim in two steps.

First, we show that $\hat{S}_{m} \subseteq S_{m}^{*}$. To see why, notice that Lemma $5 \operatorname{implies} \hat{G}_{m}(s) \leq G_{m}^{*}(s)$, so the lowest score in $\hat{S}_{m}$ is not lower than the lowest score in $S_{m}^{*}$. Moreover, we show in Part I that $\bar{s}_{m}^{*}$ is the largest score in $\hat{S}_{m}$, so we have $\hat{S}_{m} \subseteq\left[\underline{s}_{m}^{*}, \bar{s}_{m}^{*}\right]$. Then, Proposition 4 implies that gaps in $S_{m}^{*}$ are not in $\hat{S}_{m}$, so $\hat{S}_{m} \subseteq S_{m}^{*}$.

Second, we show that, for any $s \in \hat{S}_{m}$, player $m+1$ 's payoff at $s$ against $\hat{G}_{1}, \ldots, \hat{G}_{m}$ cannot be higher than $u_{m+1}^{*}$. To see why, take any $s \in \hat{S}_{m}$, we have $s \in S_{m}^{*}$, so $m$ is active at $s$. By the definition of equilibrium, player $m+1$ 's payoff at $s$ against $G_{1}^{*}, \ldots, G_{m}^{*}$ cannot be higher than $u_{m+1}^{*}$. In addition, Lemmas 11 and 14 in the supplementary note imply that player $m+1$ 's payoff at $s$ against $\hat{G}_{1}, \ldots, \hat{G}_{m}$ is lower than his payoff at $s$ against $G_{1}^{*}, \ldots, G_{m}^{*}$. Therefore, the second step is proved.

Parts I and II complete the proof.

\section{B Algorithm Properties}

This appendix provides proofs for the results in Section 3.2.

Proof of Proposition 6 (Finiteness). By exactly the same proof for Lemma 8, we can show that the pseudo strategy $\hat{G}_{i}^{j}$ in Step 3 has at most a finite number of dents in its support. Therefore, the description of the algorithm implies that it ends in a finite number of steps.

Proof of Proposition 7 (Determinateness). It is sufficient to show that $\bar{G}_{i}^{j}$ is a function of $\bar{s}-s$. First consider $\bar{G}_{1}^{2}$ and $\bar{G}_{2}^{2}$. For $i=1$ and 2 , we have $u_{i}=v^{1}-c_{i} \bar{s}$, so $u_{i}+c_{i} s=v^{1}-c_{i}(\bar{s}-s)$, 
which is a function of $\bar{s}-s$. Since $\bar{G}_{1}^{2}$ and $\bar{G}_{2}^{2}$ are defined by

$$
\begin{aligned}
& W\left(G_{2}, v^{1}, v^{2}\right)=u_{1}+C_{1}(s)=v^{1}-c_{1}(\bar{s}-s) \\
& W\left(G_{1}, v^{1}, v^{2}\right)=u_{2}+C_{2}(s)=v^{1}-c_{2}(\bar{s}-s)
\end{aligned}
$$

$\bar{G}_{1}^{2}$ and $\bar{G}_{2}^{2}$ are also functions of $\bar{s}-s$.

Now we proceed to $\bar{G}_{1}^{3}, \bar{G}_{2}^{3}$ and $\bar{G}_{3}^{3}$. According to the algorithm,

$$
u_{3}+c_{3} \bar{s}=\max _{s \in \hat{S}_{2}^{2}} W\left(\bar{G}_{1}^{2}, \bar{G}_{2}^{2}, v^{1}, v^{2}, v^{3}\right)+c_{3}(\bar{s}-s)
$$

Since $\bar{G}_{1}^{2}$ and $\bar{G}_{2}^{2}$ are functions of $\bar{s}-s$, the right hand side is a function of $\bar{s}-s$, and the maximum $u_{3}+c_{3} \bar{s}$ is independent of $\bar{s}$. Therefore, $u_{3}+c_{3} s=u_{3}+c_{3} \bar{s}-c_{3}(\bar{s}-s)$ is a function of $\bar{s}-s$, so $u_{i}+c_{i} s$ for $i=1,2,3$ is a function of $\bar{s}-s$. We can rewrite (6) to (8) as

$$
\begin{aligned}
& W\left(\bar{G}_{2}^{3}, \bar{G}_{3}^{3}, v^{1}, v^{2}, v^{3}\right)=u_{1}+c_{1} s \\
& W\left(\bar{G}_{1}^{3}, \bar{G}_{3}^{3}, v^{1}, v^{2}, v^{3}\right)=u_{2}+c_{2} s \\
& W\left(\bar{G}_{1}^{3}, \bar{G}_{2}^{3}, v^{1}, v^{2}, v^{3}\right)=u_{3}+c_{3} s
\end{aligned}
$$

where the right hand sides are functions of $\bar{s}-s$. The algorithm defines $\bar{G}_{1}^{3}, \bar{G}_{2}^{3}, \bar{G}_{3}^{3}$ as the solution to the above equations, so they are functions of $\bar{s}-s$ as well.

Similarly, at the end of Step 1. $(m+1)$, pseudo strategies $\bar{G}_{1}^{m+1}, \ldots, \bar{G}_{m+1}^{m+1}$ are also functions of $\bar{s}-s$. Therefore, $\left(\hat{G}_{i}^{*}\right)_{i \in \mathcal{N}}$ is independent of the initial value of $\bar{s}$, and the algorithm uniquely determines $\left(\hat{G}_{i}^{*}\right)_{i \in \mathcal{N}}$.

\section{Proof of Proposition 9}

We first show that $\bar{s}_{i}$ defined in Step $1 . i$ is the infimum of maximizers obtaining the maximum $u_{i}$. By the definition of $\bar{G}_{1}^{i}, \ldots, \bar{G}_{i}^{i}$, player $i$ 's payoff at $\bar{s}_{i}$ against $\bar{G}_{1}^{i}, \ldots, \bar{G}_{i-1}^{i}$ is $u_{i}$. Since $\bar{G}_{j}^{i-1}\left(\bar{s}_{1}\right)=\bar{G}_{j}^{i}\left(\bar{s}_{i}\right)$ for $j=1, \ldots, i-1$, player $i$ 's payoff at $\bar{s}_{i}$ against $\bar{G}_{1}^{i-1}, \ldots, \bar{G}_{i-1}^{i-1}$ is also $u_{i}$. Hence, $\bar{s}_{i}$ is one of the maximizers. Now we show that $\bar{s}_{i}$ is the infimum of maximizers. Suppose there is $\bar{s}_{i}^{\prime}<\bar{s}_{i}$ and $\bar{s}_{i}$ also gives $i$ a payoff of $u_{i}$ against $\bar{G}_{1}^{i-1}, \ldots, \bar{G}_{i-1}^{i-1}$. By the same agreement in the proof of Proposition 5 , Lemmas 11 and 14 in the supplementary note imply that $i$ 's payoff at $\bar{s}_{i}^{\prime}$ against $\bar{G}_{1}^{i-1}, \ldots, \bar{G}_{i-1}^{i-1}$ is lower than $u_{i}$. Therefore, $\bar{s}_{i}^{\prime}$ is not a maximizer, which is a contradiction.

Now we proceed to show that $\bar{s}_{i}$ remains the same or decreases if $c_{i}$ increases. To see that, first consider the case in which $\bar{s}_{i}$ is an interior maximizer. Then, it satisfies the first order condition

$$
\partial W\left(\bar{G}_{1}^{i-1}(s), \ldots, \bar{G}_{i-1}^{i-1}(s), v^{1}, \ldots, v^{i}\right) / \partial s-c_{i}=0
$$

and the second order condition requires that $\partial W\left(\bar{G}_{1}^{i-1}(s), \ldots, \bar{G}_{i-1}^{i-1}(s), v^{1}, \ldots, v^{i}\right) / \partial s$ is non-decreasing in $s$. Therefore, if $c_{i}$ increases, the interior maximizer does not increase. Second, consider the case in which $\bar{s}_{i}$ is a maximizer on the boundary. If $c_{i}$ increases, the left hand side of (57) decreases, so 
$\bar{s}_{i}$ does not increase either. The two cases imply that $\bar{s}_{i}$ does not decrease if $c_{i}$ increases, so $\bar{s}_{i-1}-\bar{s}$ remains the same or increases. After Step 1.i, the difference $\bar{s}_{i-1}-\bar{s}_{i}$ remains the same because we only shift the pseudo strategies in Step 2 . As a result, if $c_{i}$ increases, $\bar{s}_{i-1}-\bar{s}_{i}$ remains the same or increases in the equilibrium.

Now let us consider the extreme cases. If the cost sequence is convex enough, $c_{i}$ is large enough relative to $c_{1}, \ldots, c_{i-1}$ so player $i$ 's best response in $\hat{S}_{i-1}$ against $\bar{G}_{1}^{i-1}, \ldots, \bar{G}_{i-1}^{i-1}$ is at $\underline{s}_{i-2}$, the lower

boundary of $\hat{S}_{i-1}$. Therefore, the minimum score in $G_{i}$ 's support is the maximum score in $G_{i+2}$ 's support. Consider another extreme case in which the costs are very similar. In particular, if $c_{i}$ is very close to $c_{i-1}$, player $i$ 's best response in $\hat{S}_{i-1}$ against $\bar{G}_{1}^{i-1}, \ldots, \bar{G}_{i-1}^{i-1}$ is at the upper boundary $\bar{s}_{i-1}$ in Step 1.i. Therefore, the maximum scores in $G_{i}$ and $G_{i+1}$ 's supports are the same. Similarly, all the players' strategies have the same maximum score in their supports.

\section{Nonlinear Costs}

This appendix contains the proofs for Section 5. First, we can verify that all the results except Lemma 6 are also true for nonlinear costs. Therefore, given any equilibrium, if the algorithm starts with $\bar{s}_{1}^{*}$ - the upper bound of player 1's equilibrium strategy's support, the algorithm constructs the equilibrium. Moreover, we have the following lemma.

Lemma 9 Fix any equilibrium and let $\bar{s}_{1}^{*}$ be the upper bound of all supports of the equilibrium strategies. Then, i) if the algorithm starts with $\bar{s}>\bar{s}_{1}^{*}$, we have $\hat{u}_{i}^{*}<u_{i}^{*}$ for $i=1, \ldots, m+1$; and ii) if the algorithm starts with $\bar{s}<\bar{s}_{1}^{*}$, we have $\hat{u}_{i}^{*}>u_{i}^{*}$ for $i=1, \ldots, m+1$.

Proof. We use induction to prove. Suppose $\bar{s}>\bar{s}_{1}^{*}$.

First, we will show that $u_{2}<u_{2}^{*}$ and $\underline{s}_{2}>\underline{s}_{2}^{*}$. Since $\bar{s}_{1}>\bar{s}_{1}^{*}, u_{1}<u_{1}^{*}$ and $u_{2}<u_{2}^{*}$. Player 2 's payoff at $\underline{s}_{2}$ should be

$$
0-C_{2}\left(\underline{s}_{2}\right)=u_{2}<u_{2}^{*}=0-C_{2}\left(\underline{s}_{2}^{*}\right)
$$

so $\underline{s}_{2}>\underline{s}_{2}^{*}$.

Second, suppose $u_{l}<u_{l}^{*}$, we want to show that $\underline{s}_{l+1}>\underline{s}_{l+1}^{*}$ and $u_{l+1}<u_{l+1}^{*}$. In particular, since $u_{l}<u_{l}^{*}$, when we construct pseudo strategies for $1, \ldots, l+1$, player l's payoff at $\underline{s}_{l}$ should be

$$
v^{l+1}-C_{l}\left(\underline{s}_{l}\right)=u_{l}<u_{l}^{*}=v^{l+1}-C_{l}\left(\underline{s}_{l}^{*}\right)
$$

Therefore $\underline{s}_{l}>\underline{s}_{l}^{*}$. Then, we have $\underline{s}_{l+1}>\underline{s}_{l+1}^{*}$ because $\underline{s}_{l}=\underline{s}_{l+1}$ and $\underline{s}_{l}^{*}=\underline{s}_{l+1}^{*}$. Player $l+1$ 's payoff at $\underline{s}_{l+1}$ should be:

$$
u_{l+1}=v^{l+1}-C_{l+1}\left(\underline{s}_{l+1}\right)<v^{l+1}-C_{l+1}\left(\underline{s}_{l+1}^{*}\right)=u_{l+1}^{*}
$$

Therefore, induction implies $\underline{s}_{m}>\underline{s}_{m}^{*}$. Moreover, $u_{i}<u_{i}^{*}$ for $i=1, \ldots, m+1$.

Similarly, if $\bar{s}_{1}<\bar{s}_{1}^{*}$, we have $u_{i}>u_{i}^{*}$ for all $i$ and $\underline{s}_{m}<\underline{s}_{m}^{*}$.

Proof of Theorem 1 for Nonlinear Costs. If we replace Lemma 6 with Lemma 9, the proof of Theorem 1 for linear costs also works for nonlinear costs. Hence, we have completed the proof 
of Theorem 1.

If the algorithm starts with $\bar{s}_{1}^{*}$, Lemma 9 ensures that the algorithm for nonlinear costs finds the lower supports of the equilibrium strategies. Similar to the analysis for linear costs, the algorithm constructs the unique equilibrium if it starts with $\bar{s}_{1}^{*}$.

Proof of Theorem 3. If $\bar{s}>\bar{s}_{1}^{*}$, Lemma 9 implies that $\bar{s}_{1}^{*}$ is in the interval $\left[\bar{s}, \bar{s}^{u}\right]$. Since $\bar{s}$ is also in the same interval, and the interval shrinks by half after each iteration, we have $\left|\bar{s}-\bar{s}_{1}^{*}\right|=O\left(2^{-T}\right)$. Similarly, $\left|u_{i}-u_{i}^{*}\right|=O\left(2^{-T}\right)$ for all $i$.

Now we will show that $\left|\hat{G}_{i}^{*}(s)-G_{i}^{*}(s)\right|=O\left(2^{-T}\right)$ for each $i$ and $s$. Given $u_{1}, \ldots, u_{i}$, we construct the pseudo strategies $\bar{G}_{1}^{i}, \ldots, \bar{G}_{i}^{i}$. In particular, we first define $\bar{G}_{i-1}^{i}$ and $\bar{G}_{i}^{i}$ as the solution to

$$
\begin{aligned}
W\left(\bar{G}_{i-1}^{i}(s), v^{i-1}, v^{i}\right)-C_{i-1}(s) & =u_{i-1} \\
W\left(\bar{G}_{i}^{i}(s), v^{i-1}, v^{i}\right)-C_{i}(s) & =u_{i}
\end{aligned}
$$

Similar to (47), the solution to (58) and (59) also solves the ordinary differential equation system

$$
\left(\begin{array}{c}
\bar{G}_{i-1}^{i \prime} \\
\bar{G}_{i}^{i \prime}
\end{array}\right)=\left(\begin{array}{cc}
0 & v^{i-1}-v^{i} \\
v^{i-1}-v^{i} & 0
\end{array}\right)^{-1}\left(\begin{array}{c}
C_{i-1}^{\prime} \\
C_{i}^{\prime}
\end{array}\right)
$$

with the initial conditions

$$
\begin{aligned}
\bar{G}_{i-1}^{i}\left(\underline{s}_{i}\right) v^{i-1}+\left(1-\bar{G}_{i-1}^{i}\left(\underline{s}_{i}\right)\right) v^{i}-C_{i-1}\left(\underline{s}_{i}\right) & =u_{i-1} \\
\bar{G}_{i}^{i}\left(\underline{s}_{i}\right) & =0
\end{aligned}
$$

Since $\left|u_{i}-u_{i}^{*}\right|=O\left(2^{-T}\right)$, we have $\left|\underline{s}_{i}-\underline{s}_{i}^{*}\right|=O\left(2^{-T}\right)$ where $\underline{s}_{i}^{*}$ is the counterpart of $\underline{s}_{i}$ if the algorithm starts with $\bar{s}_{1}^{*}$. Let $\bar{G}_{i}^{i *}(s)$ be the counterpart of $\bar{G}_{i}^{i}(s)$ if the algorithm starts with $\bar{s}_{1}^{*}$. Notice that the domains of pseudo strategies $\bar{G}_{i-1}^{i}$ and $\bar{G}_{i-1}^{i}$ are bounded, so we have $\left|\bar{G}_{i}^{i \prime}(s)-\bar{G}_{i}^{i * \prime}(s)\right|=$ $O\left(2^{-T}\right)$ and $\left|\bar{G}_{i}^{i}(s)-\bar{G}_{i}^{i *}(s)\right|=O\left(2^{-T}\right)$. Similarly, all the pseudo strategies defined in Step $1 . i$ satisfy $\left|\bar{G}_{j}^{i}(s)-\bar{G}_{j}^{i *}(s)\right|=O\left(2^{-T}\right)$.

In Step 3.i, pseudo strategy $\hat{G}_{i}^{i}(s)$ is replaced by the smallest monotone function $\hat{G}_{i}^{*}(s)$ that lies on or above it. It can be verified that, after this step, we still have $\left|\hat{G}_{i}^{*}(s)-\hat{G}_{i}^{+}(s)\right|=O\left(2^{-T}\right)$, where $\hat{G}_{i}^{+}(s)$ is the counterpart of $\hat{G}_{i}^{*}(s)$ if the algorithm starts with $\bar{s}_{1}^{*}$. Similarly, we have $\left|\hat{G}_{i}^{*}(s)-\hat{G}_{i}^{+}(s)\right|=O\left(2^{-T}\right)$ for each $i$ and $s$ at the end of Step 3.

Proof of Corollary 1. Part i) of the corollary comes from the participation property in Section 3.1. Then, it remains to show part ii). Suppose $C_{i}(s)$ pointwise converges to $C_{i+1}(s)$. Let us consider the equilibrium in the limit. Proposition 2 implies $\bar{s}_{i}^{*} \geq \bar{s}_{i+1}^{*}$. Suppose $\bar{s}_{i}^{*}>\bar{s}_{i+1}^{*}$, therefore $i$ 's expected winnings at $\bar{s}_{i+1}^{*}$ are more than $i+1$ 's, therefore $i+1$ would deviate to $\bar{s}_{i}^{*}$ for a higher payoff. Then $\bar{s}_{i}^{*}=\bar{s}_{i+1}^{*}$, and the payoffs of $i$ and $i+1$ are also the same. 
From the way we construct the strategies for $i$ and $i+1$, their strategies $G_{i}^{*}(s)$ and $G_{i+1}^{*}(s)$ must also converge at any $s$ in the common supports.

\section{E Applications}

\section{E.1 Tracking}

Proof of Proposition 10. Let us derive the total effort if the students are tracked. According to the algorithm, their payoffs are the lowest prize $u_{H}=u_{L}=v^{2 n}$, which is 0 in a QPS and positive in a GPS. Denote the total prize in each classroom as $V=v^{1}+\ldots+v^{2 n}$. For each class, the total prize less the total cost equals the total payoff, so the total effort is $\left(V-2 n u_{H}\right) / c_{H}$ in the classroom of $H$-type students and $\left(V-2 n u_{L}\right) / c_{L}$ in the other. Hence the total effort under tracking is

$$
\Pi_{\text {track }}=\left(V-2 n v^{2 n}\right) / c_{H}+\left(V-2 n v^{2 n}\right) / c_{L}
$$

Now consider the case in which the students are mixed. We first show five claims then combine them to prove the proposition.

First, we claim that $\bar{s}_{H}=\bar{s}_{L}$ or $\bar{s}_{L}=\underline{s}_{H}$, which means either all the equilibrium strategies' supports have the same upper bound, or the supports of different players' strategies do not overlap. Recall that in the proof of Proposition 9, we show that $\bar{s}_{i}$ defined in Step 1.i is the infimum of maximizers obtaining the maximum $u_{i}$. As a result, it is sufficient to show that

$W\left(\mathbf{G}_{H}(s), v^{1}, \ldots, v^{n+1}\right)-c_{L} s$ is convex, where $\mathbf{G}_{H}(s)$ is a $n$-dimension vector with the same entry $G_{H}(s)$. First, consider QPS. The contest with only $n H$-type students has an equilibrium with symmetric strategies $G_{H}$ that solve

$$
\beta\left(G_{H}(s)\right)^{2}(n-1)(n-2) / 2+\left(v^{n-1}-v^{n}\right)(n-1) G_{H}(s)+v^{n}-c_{H} s=v^{n}
$$

Taking second order derivatives both sides w.r.t $s$, we have

$$
\beta(n-1)(n-2)\left[G_{H}(s) g_{H}^{\prime}(s)+\left(g_{H}(s)\right)^{2}\right]+\left(v^{n-1}-v^{n}\right)(n-1) g_{H}^{\prime}(s)=0
$$

We claim that $g_{H}^{\prime}<0$, otherwise the left hand side of the equation above is positive. Consider an $L$-type player's best response against $n H$-type students with $G_{H}$. His payoff at score $s$ is

$$
\beta\left(\left(G_{H}(s)\right)^{2} n(n-1) / 2\right)+\left(v^{n}-v^{n+1}\right) n G_{H}(s)+v^{n+1}-c_{L} s
$$

whose second order derivative w.r.t. $s$ is

$$
\begin{aligned}
& \left.\beta n(n-1)_{H}(s) g_{H}^{\prime}(s)+\left(g_{H}(s)\right)^{2}\right]+\left(v^{n}-v^{n+1}\right) n g_{H}^{\prime}(s) \\
= & \left\{\begin{array}{cl}
-\frac{1}{n-2}\left(v^{2 n-1}+2(n-1) \beta\right) n g_{H}^{\prime}(s)>0 & \text { if } n>2 \\
2 \beta\left(g_{H}(s)\right)^{2}>0 & \text { if } n=2
\end{array}\right.
\end{aligned}
$$


where the equality comes from (61) and the inequality is implied by $g_{H}^{\prime}(s)>0$. Hence, the payoff of an $L$-type player is convex in $s$ if he faces $n H$-type students with strategy $G_{H}$. Then the algorithm implies that there could be only two possibilities: $\bar{s}_{H}=\bar{s}_{L}$ or $\bar{s}_{L}=\underline{s}_{H}$. Now let us consider GPS. The contest with only $n H$-type students has symmetric strategies $G_{H}$ that solve

$$
v^{n}\left(\alpha G_{H}(s)+\left(1-G_{H}(s)\right)\right)^{n-1}-c_{H} s=v^{n}
$$

Then the best response of an $L$-type player against $n H$-type students with strategy $G_{H}$ maximizes

$$
v^{n+1}\left(\alpha G_{H}(s)+\left(1-G_{H}(s)\right)\right)^{n}-c_{L} s
$$

We can solve $G_{H}$ from (62) and substitute it into the payoff above, then we can verify that the payoff is convex in $s$. Therefore the best responses can only be at boundaries, so we must have $\bar{s}_{H}=\bar{s}_{L}$ or $\bar{s}_{L}=\underline{s}_{H}$.

Second, if $\alpha=1$ or $\beta=0$, the prize sequence is linear, and we have $\Pi_{\text {mix }} \leq \Pi_{\text {track }}$. Consider the QPS first. Denote $v^{i}-v^{i+1}=\theta \geq 0$ for all $i=1, \ldots, m$, so $v^{i}=\theta(2 n-i)$. If there are only $H$-type students in the contests, the algorithm implies the equilibrium strategies are symmetric and $G_{H}(s)$ is linear. As a result, the algorithm also implies that the highest score $\bar{s}_{L}$ in the support of equilibrium strategy $G_{L}$ is either the highest score $\bar{s}_{H}$ or the lowest score $\underline{s}_{H}$ in the support of $G_{H}(s)$. Consider the first possibility with $\bar{s}_{L}=\underline{s}_{H}$. As above, we also have $u_{L}=0$, $v^{n+1}-c_{L} \bar{s}_{L}=u_{L}$ and $u_{H}=v^{n}-c_{H} \bar{s}_{L}$. Then we can solve for $\bar{s}_{L}$, and the payoff of $H$-type players can be rewritten as $u_{H}=v^{n}+v^{n+1} c_{H} / c_{L}$. The total value of prizes won by $H$-type students in one classroom is

$$
V_{H}=v^{1}+\ldots+v^{n}=(n \theta+(2 n-1) \theta) n / 2
$$

and the total value of prizes won by $L$-type students is

$$
V_{L}=v^{n+1}+\ldots+v^{2 n}=(n-1) \theta n / 2
$$

Total effort with mixed students is

$$
\Pi_{m i x}=2\left(V_{H}-n u_{H}\right) / c_{H}+2\left(V_{L}-n u_{L}\right) / c_{L}
$$

Since the prize sequence is arithmetic, the total prize value is $V=\theta(2 n-1) n$. Substituting the expressions of $u_{H}, u_{L}, V, V_{H}$ and $V_{L}$ derived above into the difference below, we have

$$
\begin{aligned}
\Pi_{\text {mix }}-\Pi_{\text {track }} & =2\left(V_{H}-n u_{H}\right) / c_{H}+2\left(V_{L}-n u_{L}\right) / c_{L}-\left(V / c_{H}+V / c_{L}\right) \\
& =-n \frac{\theta}{c_{H} c_{L}}\left(2 c_{H}+n\left(c_{L}-c_{H}\right)\right)<0
\end{aligned}
$$

Note that, if a GPS is linear, the $2 n$ prizes are the same in each classroom. Therefore, the total effort would be 0 with either mixing or tracking, and $\Pi_{m i x}=\Pi_{\text {track }}$.

Third, if $\alpha$ in GPS or $\beta$ in QPS is large enough, we have $\Pi_{m i x} \geq \Pi_{\text {track }}$. If the prize sequence 
is convex enough, Proposition 9 implies that the supports of $G_{H}$ and the supports of $G_{L}$ do not overlap. That is, $\bar{s}_{L}=\underline{s}_{H}$. By choosing $\bar{s}_{L}$, an $L$-type player wins prize $v^{n+1}$ with probability one, so his payoff at $\bar{s}_{L}$ is $v^{n+1}-c_{L} \bar{s}_{L}=u_{L}$. Similarly, at score $\bar{s}_{L}$, a $H$-type player wins prize $v^{n}$ with probability one, so $u_{H}=v^{n}-c_{H} \bar{s}_{L}$. Then the prizes won by the $H$-type players are $v^{1}, \ldots, v^{n}$, and those won by the $L$-type players are $v^{n+1}, \ldots, v^{2 n}$. Since the total prize won by $H$ type players less their total costs equals their total payoff, the total score of $2 n H$-type players is $2\left(v^{1}+\ldots+v^{n}-n u_{H}\right) / c_{H}$. As a result, the total effort of all players is

$$
\Pi_{\text {mix }}=2\left(v^{1}+\ldots+v^{n}-n u_{H}\right) / c_{H}+2\left(v^{n+1}+\ldots+v^{2 n}-n u_{L}\right) / c_{L}
$$

If QPS or GPS is convex enough, $v^{1}+\ldots+v^{n}$ converges to $V$ while $v^{n}+\ldots+v^{2 n}$ converges to 0 . Since $v^{2 n}$ and $v^{n}$ also converge to 0 , both $u_{H}$ and $u_{L}$ go to 0 . As a result, $\Pi_{\text {mix }}$ converges to $2 V / c_{H}$ while $\Pi_{\text {track }}$ converges to $V / c_{H}+V / c_{L}$, so $\Pi_{\text {mix }}>\Pi_{\text {track }}$.

Fourth, if $\bar{s}_{L}=\bar{s}_{H}$, we have $\Pi_{m i x} \geq \Pi_{\text {track }}$. If $\bar{s}_{L}=\bar{s}_{H}$, they must equal $\left(v^{1}-v^{2 n}\right) / c_{L}$. Therefore, $u_{H}=v^{1}-c_{H} \bar{s}_{H}=v^{1}-\left(v^{1}-v^{2 n}\right) c_{H} / c_{L}$ and $u_{L}=v^{2 n}$. Substituting the payoffs into (63), we have

$$
\Pi_{m i x}=\left(2 V_{H}-2 n v^{1}\right)\left(1 / c_{H}-1 / c_{L}\right)-4 n v^{2 n} / c_{L}+2 V / c_{L}
$$

Moreover, because $\bar{s}_{L}=\bar{s}_{H}$, the top $n$ prizes are not only won by the $H$-type players. Therefore, $2 V_{H}<2\left(v^{1}+\ldots+v^{n}\right)$, which is the total value of the top $n$ prizes in both classrooms. Substituting the inequality into (64), we have

$$
\Pi_{\text {mix }}<\left(2\left(v^{1}+\ldots+v^{n}\right)-2 n v^{1}\right)\left(1 / c_{H}-1 / c_{L}\right)-4 n v^{2 n} / c_{L}+2 V / c_{L}
$$

As a result, we have

$$
\begin{aligned}
\Pi_{\text {track }}-\Pi_{\text {mix }}> & \left(2 V-2 n v^{2 n}\right) / c_{H}+\left(2 V-2 n v^{2 n}\right) / c_{L} \\
& -\left[\left(2\left(v^{1}+\ldots+v^{n}\right)-2 n v^{1}\right)\left(1 / c_{H}-1 / c_{L}\right)-4 n v^{2 n} / c_{L}+2 V / c_{L}\right] \\
= & 2\left(V-n v^{2 n}\right) / c_{H}+2 n v^{2 n} / c_{L}+2\left[n v^{1}-v^{1}-\ldots-v^{n}\right]\left(1 / c_{H}-1 / c_{L}\right)>0
\end{aligned}
$$

Fifth, if $\bar{s}_{L}=\underline{s}_{H}$, the effort difference $\Pi_{\text {mix }}-\Pi_{\text {track }}$ is increasing in $\alpha$ or $\beta$. If the students are mixed and $\bar{s}_{L}=\underline{s}_{H}$, the first $n$ prizes are won by $H$-type students, so we have $2 V_{H}=$ $2\left(v^{1}+\ldots+v^{n}\right)$. The equilibrium payoff must satisfy $v^{n+1}-c_{L} \bar{s}_{L}=u_{L}=v^{2 n}$ and $u_{H}=v^{n}-c_{H} \bar{s}_{L}$. Then we can solve for $u_{H}$ and substitute $u_{H}$ and $V_{H}$ into

$$
\Pi_{m i x}=\left(2 V_{H}-2 n u_{H}\right) / c_{H}+\left(2 V-2 V_{H}-2 n v^{2 n}\right) / c_{L}
$$

In addition, substituting $v^{i}=(2 n-i) v^{2 n-1}+\beta \sum_{j=1}^{2 n-i-1} j$ of a QPS in (65), we can verify that $\Pi_{\text {track }}-\Pi_{\text {mix }}$ is decreasing in $\beta$ for QPS. If a GPS, $v^{i}=v^{2 n} \alpha^{2 n-i}$, similar analysis implies

$$
\Pi_{\text {mix }}=2\left(\frac{\alpha^{n}\left(n(\alpha-1) \alpha^{n-1}-\left(\alpha^{n}-1\right)\right)}{\alpha-1} \frac{1}{c_{H}}-\frac{n-n \alpha^{n-1}-n \alpha+\alpha^{n}+n \alpha^{n}-1}{\alpha-1} \frac{1}{c_{L}}\right) v^{2 n}
$$


whose derivative in $\alpha$ is less than that of

$$
\begin{aligned}
& 2\left(\frac{\alpha^{n}\left(n(\alpha-1) \alpha^{n-1}-\left(\alpha^{n}-1\right)\right)}{\alpha-1}-\frac{n-n \alpha^{n-1}-n \alpha+\alpha^{n}+n \alpha^{n}-1}{\alpha-1}\right) \frac{1}{c_{L}} v^{2 n} \\
= & \frac{2}{\alpha-1}\left[n(\alpha-1) \alpha^{2 n-1}-n(\alpha-1) \alpha^{n-1}+n(\alpha-1)-\left(\alpha^{2 n}-1\right)\right] v^{2 n} / c_{L} \\
= & 2\left(n \alpha^{2 n-1}+n \alpha^{n-1}+n-\alpha^{2 n-1}-\alpha^{2 n-2}-\ldots-1\right) v^{2 n} / c_{L} \\
= & 2\left[\left(n \alpha^{2 n-1}-\alpha^{2 n-1}-\ldots-\alpha^{2 n-n}\right)+\left(n \alpha^{n-1}-\alpha^{n-1}-\ldots-1\right)+n\right] v^{2 n} / c_{L}
\end{aligned}
$$

Since the above expression is increasing in $\alpha$, we must have that $\Pi_{m i x}$ increases in $\alpha$ and $\Pi_{\text {track }}-$ $\Pi_{\text {mix }}$ decreases in $\alpha$.

Now let us combine the five claims to prove the proposition. The first claim implies that $\bar{s}_{H}=\bar{s}_{L}$ or $\bar{s}_{L}=\underline{s}_{H}$. On the one hand, suppose $\bar{s}_{H}=\bar{s}_{L}$ if $\beta=0$. According to Proposition 9, there exists $\beta^{\prime}>0$ such that $\bar{s}_{H}=\bar{s}_{L}$ if $\beta \geq \hat{\beta}$ and $\underline{s}_{H}=\bar{s}_{L}$ otherwise. The fourth claim implies that $\Pi_{m i x}<\Pi_{\text {track }}$ if $\beta \leq \hat{\beta}$, the third claim implies $\Pi_{\text {mix }}>\Pi_{\text {track }}$ if $\beta$ is large. Then, the fifth claim implies that there exists $\beta^{\prime} \geq \hat{\beta}$ such that $\Pi_{\text {mix }} \leq \Pi_{\text {track }}$ if $\beta \leq \hat{\beta}^{\prime}$ and $\Pi_{\text {mix }}>\Pi_{\text {track }}$ otherwise.

Hence, $\Pi_{\text {mix }}>\Pi_{\text {track }}$ if and only if $\beta>\hat{\beta}^{\prime}$. On the other hand, suppose $\bar{s}_{L}=\underline{s}_{H}$ if $\beta=0$. The second claim implies that $\Pi_{\text {mix }} \leq \Pi_{\text {track }}$ if $\beta=0$, and the third claim implies $\Pi_{\text {mix }}>\Pi_{\text {track }}$ if $\beta$ is large. Then, the fifth claim implies that there exists $\beta^{\prime} \geq \hat{\beta}$ such that $\Pi_{\text {mix }}>\Pi_{\text {track }}$ if and only if $\beta>\hat{\beta}^{\prime}$. Similarly, there exists $\alpha^{\prime} \geq 1$ such that $\Pi_{\text {mix }}>\Pi_{\text {track }}$ if and only if $\alpha>\alpha^{\prime}$.

\section{E.2 Winner-Take-All}

Proof of Proposition 11. On the one hand, consider the case with only one prize. Then only the strongest two players are active, and their equilibrium strategies $G_{1}$ and $G_{2}$ satisfy

$$
\begin{aligned}
G_{2}(s)-c_{1} s & =1-c_{1} / c_{2} \\
G_{1}(s)-c_{2} s & =0
\end{aligned}
$$

The total winnings of player 1 is $\hat{W}_{1}=\int_{0}^{1 / c_{2}} G_{2}(s) d G_{1}(s)=1-c_{1} /\left(2 c_{2}\right)$, and the total winnings of player 2 is $\hat{W}_{2}=c_{1} /\left(2 c_{2}\right)$. Since the payoffs are $u_{1}=1-c_{1} / c_{2}$ for 1 and $u_{2}=0$ for 2 , the total effort is

$$
\begin{aligned}
\Pi_{W T A} & =\left(\hat{W}_{1}-u_{1}\right) / c_{1}+\hat{W}_{2} / c_{2} \\
& =\left[\left(1-c_{1} /\left(2 c_{2}\right)\right)-\left(1-c_{1} / c_{2}\right)\right] / c_{1}+\left[c_{1} /\left(2 c_{2}\right)\right] / c_{2} \\
& =\left(c_{1}+c_{2}\right) /\left(2 c_{2}^{2}\right)
\end{aligned}
$$

Therefore

$$
\lim _{c_{1} \rightarrow 0} \Pi_{W T A}=1 /\left(2 c_{2}\right)
$$

On the other hand, consider the case with multiple prizes, $v^{1}$ and $v^{2}+\ldots+v^{m}=1-v^{1}$, where $2 \leq m \leq n$. Let the sequence be either homogeneous prize sequence (HPS), GPS or QPS, so our 
algorithm applies. Then, players $1, \ldots, n^{\prime} \equiv \min (m+1, n)$ are competing in the contest while others choose zero score with probability 1 . Let $G_{1}$ be the equilibrium strategy of player 1 , then Corollary 1 implies that the players weaker than 1 use the same strategy, denote it as $G_{2}$. Moreover, the algorithm implies that $G_{1}$ and $G_{2}$ have interval supports, whose upper boundaries are the same. Let the support be $\left[\underline{s}_{1}, \bar{s}_{1}\right]$ for $G_{1}$ and $\left[0, \bar{s}_{1}\right]$ for $G_{2}$. Therefore, the upper boundary is $\bar{s}_{1}=\left(v^{1}-v^{n}\right) / c_{2}$, and $u_{1}=v^{1}-c_{1} \bar{s}_{1}, u_{2}=v^{n}$.

In the remainder of the proof, we first derive the limit of the total expected effort if $c_{1}$ converges to 0 . Then, we use the expression of the limit to prove (10). For any $s \in\left[\underline{s}_{1}, \bar{s}_{1}\right]$, the indifference condition for player 1 is $W\left(G_{2}(s), \ldots, G_{2}(s), v^{1}, \ldots, v^{m}\right)-c_{1} s=u_{1}$, where the number of $G_{2}(s)$ is $n^{\prime}-1$. If $c_{1}$ converges to $0, W\left(G_{2}(s), \ldots, G_{2}(s), v^{1}, \ldots, v^{m}\right)$ becomes constant for different $s$. Therefore, $\lim _{c_{1} \rightarrow 0} G_{2}(s)=1$ for $s \in\left[\underline{s}_{1}, \bar{s}_{1}\right]$. As a result, prizes $v^{2}, \ldots, v^{m}$ are won by players $2, \ldots, n^{\prime}$ with probability 1 . As a result, the total expected effort of players $2, \ldots, n^{\prime}$ is

$$
\lim _{c_{1} \rightarrow 0}\left(n^{\prime}-1\right) E\left[s_{2}\right]=\left(1-v^{1}-\left(n^{\prime}-1\right) u_{2}\right) / c_{2}
$$

For any $s \in\left[\underline{s}_{1}, \bar{s}_{1}\right]$, the indifference condition for player 1 is $W\left(G_{1}(s), G_{2}(s), \ldots, G_{2}(s), v^{1}, \ldots, v^{m}\right)-$ $c_{2} s=u_{2}$, where the number of $G_{2}(s)$ is $n^{\prime}-2$. Because $\lim _{c_{1} \rightarrow 0} G_{2}(s)=1$, if $c_{1}$ converges to 0 , the indifference condition becomes $v^{1} G_{1}(s)+v^{2}\left(1-G_{1}(s)\right)-c_{2} s=u_{2}$, so $\lim _{c_{1} \rightarrow 0} G_{1}(s)=$ $\left(c_{2} s+u_{2}-v^{2}\right) /\left(v^{1}-v^{2}\right)$, which is a uniform distribution over $\left[\left(v^{2}-u_{2}\right) / c_{2},\left(v^{1}-u_{2}\right) / c_{2}\right]$. Therefore, the expected effort for player 1 is

$$
\lim _{c_{1} \rightarrow 0} E\left[s_{1}\right]=\int_{\underline{s}_{1}}^{\bar{s}_{1}} s d\left(\frac{c_{2} s+u_{2}-v^{2}}{v^{1}-v^{2}}\right)=\frac{v^{1}+v^{2}+2 u_{2}}{2 c_{2}}
$$

Hence, the total expected effort for any HPS, GPS or QPS with $m \geq 2$ positive prizes satisfies

$$
\lim _{c_{1} \rightarrow 0} \Pi=\lim _{c_{1} \rightarrow 0}\left(n^{\prime}-1\right) E\left[s_{2}\right]+\lim _{c_{1} \rightarrow 0} E\left[s_{1}\right]=\left[1-\left(v^{1}-v^{2}\right) / 2-\left(n^{\prime}-2\right) v^{n}\right] / c_{2}
$$

Using (67), we prove (10) in four steps. First, $\lim _{c_{1} \rightarrow 0} \Pi_{H P S}^{*}=1 / c_{2}$. Since the total value of prizes is fixed to 1 , a HPS is characterized by $m$, the number of its positive prizes. Therefore, the total expected effort is a function of $m$, and denote it as $\Pi(m)$. Then,

$$
\lim _{c_{1} \rightarrow 0} \Pi_{H P S}^{*}=\lim _{c_{1} \rightarrow 0} \max _{m \in\{2, \ldots, n\}} \Pi(m)=\max _{m \in\{2, \ldots, n\}} \lim _{c_{1} \rightarrow 0} \Pi(m)=1 / c_{2}
$$

where the second equality is because the set $\{2, \ldots, n\}$ is finite.

Second, $\lim _{c_{1} \rightarrow 0} \Pi_{Q P S}^{*}=\frac{1}{c_{2}}\left(1-\frac{1}{n(n-1)}\right)$. Recall that a QPS is characterized by parameters $m, v^{m-1}$ and $\beta$. Because the total prize value is 1 , parameters $m$ and $v^{m}$ determine a QPS. If the prize sequence is arithmetic, $v^{m}$ is largest and takes a value of $2 /(m(m+1))$. Let $\Pi\left(m, v^{m}\right)$ be the total expected effort with a QPS. Given $m$, the algorithm implies that $\Pi(m, \cdot)$ is continuous over 
the bounded interval $[0,2 /(m(m+1))]$, so it is also uniformly continuous. Then,

$$
\begin{aligned}
\lim _{c_{1} \rightarrow 0} \Pi_{Q P S}^{*} & =\lim _{c_{1} \rightarrow 0} \max _{m \in\{2, \ldots, n\}} \sup _{v^{m} \in[0,2 /(m(1+m))]} \Pi\left(m, v^{m}\right) \\
& =\max _{m \in\{2, \ldots, n\}} \lim _{c_{1} \rightarrow 0} \sup _{v^{m} \in[0,2 /(m(1+m))]} \Pi\left(m, v^{m}\right) \\
& =\max _{m \in\{2, \ldots, n\}} \sup _{v^{m} \in[0,2 /(m(1+m))]} \lim _{c_{1} \rightarrow 0} \Pi\left(m, v^{m}\right) \\
& =\frac{1}{c_{2}}\left(1-\frac{1}{n(n-1)}\right)
\end{aligned}
$$

where the third equality comes from uniform continuity of $\Pi(m, \cdot)$, and the last equality is from (67).

Third, $\frac{1}{2 c_{2}}<\lim _{c_{1} \rightarrow 0} \Pi_{G P S}^{*}<\frac{1}{c_{2}}\left(1-\frac{1}{n(n-1)}\right)$. A GPS with fixed total value can be characterized by $\alpha$, so the total expected effort $\Pi(\alpha)$ is a function of $\alpha$. We can verify that $\lim _{c_{1} \rightarrow 0} \Pi(2)>$ $1 /\left(2 c_{2}\right)$, so

$$
\lim _{c_{1} \rightarrow 0} \Pi_{G P S}^{*} \geq \lim _{c_{1} \rightarrow 0} \Pi(2)>1 /\left(2 c_{2}\right)
$$

It remains to show that $\lim _{c_{1} \rightarrow 0} \sup _{\alpha} \Pi(\alpha)<\frac{1}{c_{2}}\left(1-\frac{1}{n(n-1)}\right)$. Consider $\alpha^{*}$ that maximizes $\lim _{c_{1} \rightarrow 0} \Pi(\alpha)$. Notice that $\lim _{\alpha \rightarrow \infty} \lim _{c_{1} \rightarrow 0} \Pi(\alpha)=1 /\left(2 c_{2}\right)$ and $\lim _{c_{1} \rightarrow 0} \Pi(2)>1 /\left(2 c_{2}\right)$, so $\alpha^{*}<\infty$. Moreover, $\lim _{c_{1} \rightarrow 0} \Pi(1)=0$, so $\alpha^{*}>1$. Therefore, $\alpha^{*} \in(1,+\infty)$. Let $\left(v^{k *}\right)_{k}$ be the GPS with $\alpha^{*}$. Because $\alpha^{*}>1, v^{1 *}-v^{2 *}$ is larger than that in the arithmetic prize sequence. Moreover, $v^{n *}>0$. Then, (67) implies that

$$
\sup _{\alpha \geq 1} \lim _{c_{1} \rightarrow 0} \Pi(\alpha)=\frac{1}{c_{2}}\left(1-\frac{v^{1 *}-v^{2 *}}{2}-(n-2) v^{n *}\right)<\frac{1}{c_{2}}\left(1-\frac{1}{n(n-1)}\right)
$$

Notice that for any $\varepsilon>0$, there exists $\hat{c}>0$ such that if $c_{1}<\hat{c}$, we have $\Pi(\alpha)<\lim _{c_{1} \rightarrow 0} \Pi(\alpha)+\varepsilon$. Moreover, $\sup _{\alpha} \Pi(\alpha) \leq \sup _{\alpha} \lim _{c_{1} \rightarrow 0} \Pi(\alpha)+\varepsilon$, which implies

$$
\lim _{c_{1} \rightarrow 0} \sup _{\alpha} \Pi(\alpha) \leq \sup _{\alpha} \lim _{c_{1} \rightarrow 0} \Pi(\alpha)+\varepsilon
$$

In addition, if $\varepsilon$ is small, (68) implies that

$$
\sup _{\alpha} \lim _{c_{1} \rightarrow 0} \Pi(\alpha)+\varepsilon<\frac{1}{c_{2}}\left(1-\frac{1}{n(n-1)}\right)
$$

Combining (69) and (70), we obtain $\lim _{c_{1} \rightarrow 0} \sup _{\alpha} \Pi(\alpha)<\frac{1}{c_{2}}\left(1-\frac{1}{n(n-1)}\right)$.

Fourth, combining the first three steps and (66), we have (10). 


\section{University Library}

\section{- M M N E R VA A gateway to Melbourne's research publications}

Minerva Access is the Institutional Repository of The University of Melbourne

Author/s:

Xiao, J

Title:

Asymmetric all-pay contests with heterogeneous prizes

Date:

2016-05

Citation:

Xiao, J. (2016). Asymmetric all-pay contests with heterogeneous prizes. Journal of Economic Theory, 163, pp.178-221. https://doi.org/10.1016/j.jet.2015.12.006.

Persistent Link:

http://hdl.handle.net/11343/123866 